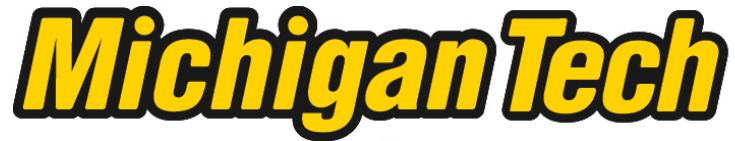 \\ Michigan Technological University Create the Future Digital Commons @ Michigan Tech
}

Dissertations, Master's Theses and Master's Reports - Open

Dissertations, Master's Theses and Master's

Reports

2011

Resource management in location aware cognitive radio networks

Suryabh Sharma

Michigan Technological University

Follow this and additional works at: https://digitalcommons.mtu.edu/etds

Part of the Electrical and Computer Engineering Commons

Copyright 2011 Suryabh Sharma

\section{Recommended Citation}

Sharma, Suryabh, "Resource management in location aware cognitive radio networks", Master's Thesis, Michigan Technological University, 2011.

https://doi.org/10.37099/mtu.dc.etds/41

Follow this and additional works at: https://digitalcommons.mtu.edu/etds

Part of the Electrical and Computer Engineering Commons 


\title{
RESOURCE MANAGEMENT IN LOCATION AWARE COGNITIVE RADIO NETWORKS
}

\author{
By \\ Suryabh Sharma
}

\begin{abstract}
A THESIS
Submitted in partial fulfillment of the requirements for the degree of MASTER OF SCIENCE

Electrical Engineering
\end{abstract}

\section{MICHIGAN TECHNOLOGICAL UNIVERSITY \\ 2011}

(C) 2011 Suryabh Sharma 
This thesis,"RESOURCE MANAGEMENT IN LOCATION AWARE COGNITIVE RADIO NETWORKS", is hereby approved in partial fulfillment of the requirements for the Degree of MASTER OF SCIENCE IN ELECTRICAL ENGINEERING.

Department of Electrical and Computer Engineering

Signatures:

Thesis Advisor

Dr. Zhi (Gerry) Tian

Department Chair

Dr. Daniel R. Fuhrmann

Date 
This is dedicated to my Grand Mom (Dadi ma). 


\section{Contents}

Contents $\ldots \ldots \ldots \ldots \ldots \ldots \ldots \ldots$ iv

List of Figures $\ldots . \ldots \ldots \ldots \ldots \ldots \ldots$. . . . . . . . . . . .

List of Tables ........................ ix

Acknowledgments ..................... $x$

Abstract. ........................... xi

1 The Beginning $\ldots \ldots \ldots \ldots \ldots \ldots \ldots \ldots \ldots \ldots \ldots$

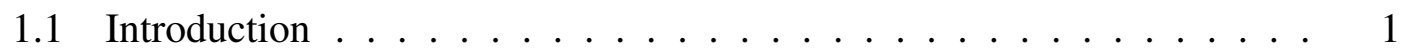

1.1 .1 Dynamic Spectrum Access (DSA) $\ldots \ldots \ldots \ldots \ldots . \quad 2$

$\begin{array}{lll}1.1 .2 & \text { Cognitive Radio Networks } \ldots \ldots \ldots \ldots \ldots \ldots & \ldots\end{array}$

1.2 Research Challenges and Motivations $\ldots \ldots \ldots \ldots$. . . . . . . 4

1.3 Research Contributions $\ldots \ldots \ldots \ldots \ldots \ldots \ldots \ldots \ldots$

1.4 Outline of the Thesis $\ldots \ldots \ldots \ldots \ldots \ldots \ldots \ldots$

2 Background and Literature Review $\ldots \ldots \ldots \ldots \ldots \ldots$. 6

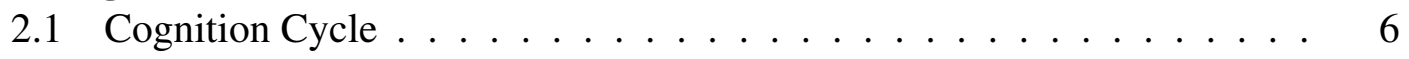

2.1 .1 Spectrum Sensing . . . . . . . . . . . . . . . . . 7

2.1 .2 Channel Identification $\ldots \ldots \ldots \ldots \ldots \ldots \ldots \ldots$

2.1 .3 Resource Management/Spectrum Management $\ldots \ldots \ldots . \quad 9$

2.2 Location Awareness . . . . . . . . . . . . . . . . . . . . . . . . . . . . . . . 10

$2.2 .1 \quad$ Full Location Aware (FLA) $\ldots \ldots \ldots \ldots \ldots \ldots \ldots$

2.2 .2 Proposed Method of PR Location Estimation $\ldots \ldots \ldots . \quad 16$

2.2 .3 Partial Location aware CRNs $\ldots \ldots \ldots \ldots \ldots \ldots$

2.2 .4 Non location Aware CRNs $\ldots \ldots \ldots$

3 System Model . . . . . . . . . . . . . . . . . . 22

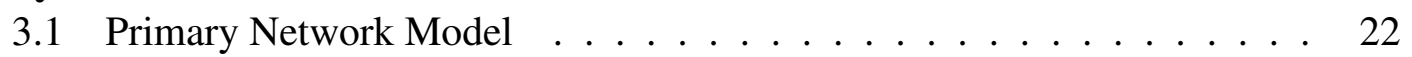

3.2 Secondary Network Model $\ldots \ldots \ldots \ldots \ldots \ldots$ 
4 Resource Management in CRNs - Single CR User . . . . . . . . . . . 25

4.1 System Setup and Problem Statement . . . . . . . . . . . . . . . 26

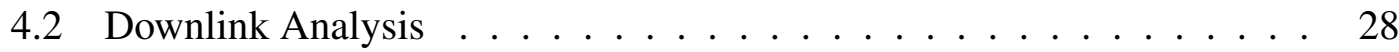

$4.2 .1 \quad$ Location Aware Scenario (LAS) $\ldots \ldots \ldots \ldots$

$4.2 .2 \quad$ Location Unaware Scenario (LUS) . . . . . . . . . . . . . . . 31

4.3 Uplink Analysis . . . . . . . . . . . . . . . . . . . . . 32

4.3 .1 Location Aware Scenario . . . . . . . . . . . . . . . . . . 33

4.4 Numerical Results and Discussion $\ldots \ldots \ldots \ldots \ldots \ldots$

$4.4 .1 \quad$ Simulation setup . . . . . . . . . . . . . . . . 34

$4.4 .2 \quad$ Effect of System Capacity and Detection Threshold . . . . . . . 34

$4.4 .3 \quad$ ATPC Parameters . . . . . . . . . . . . . . . . . . . . 36

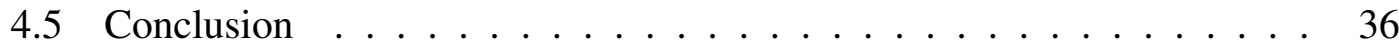

5 Resource Management in CRNs - Multi CR User $\ldots \ldots \ldots . . . . .39$

5.1 System Model and Problem Statement $\ldots \ldots \ldots$. . . . . . . . . 40

5.2 Sum Rate Maximization. . . . . . . . . . . . . . . . . . . . . 44

5.3 Hungarian Algorithm $\ldots \ldots \ldots \ldots \ldots \ldots \ldots \ldots$

5.3.1 Sum Rate Maximization based on Hungarian Algorithm . . . . 46

5.4 Maximization of Admitted Users (MAU) $\ldots \ldots \ldots \ldots \ldots \ldots$

5.4.1 Maximization of Admitted Users based on Hungarian Algorithm 52

5.5 Numerical Results . . . . . . . . . . . . . . . . . . . 54

$5.5 .1 \quad$ Simulation Setup . . . . . . . . . . . . . . . . 54

5.6 Numerical Results for Sum Rate Maximization . . . . . . . . . . . 54

5.6 .1 Effect of Number of Users . . . . . . . . . . . . . 55

5.6 .2 Effect of distance between CR pairs . . . . . . . . . . . 56

5.6 .3 Effect of Propagation Environment . . . . . . . . . . . 58

$5.6 .4 \quad$ Effect of Minimum Rate $\ldots \ldots \ldots \ldots \ldots$

5.6 .5 Effect of Duty Cycle . . . . . . . . . . . . . . 61

$5.7 \quad$ Numerical Results for Maximizing Number of Admitted Users $\ldots \ldots .63$

5.7 .1 Effect of Number of Users $\ldots \ldots \ldots \ldots$

5.7 .2 Effect of distance between CR pairs . . . . . . . . . . . 64

5.7 .3 Effect of Propagation Environment . . . . . . . . . . . . 64

$5.7 .4 \quad$ Effect of Minimum Rate $\ldots \ldots \ldots \ldots$

5.7 .5 Effect of Duty Cycle $\ldots \ldots \ldots \ldots \ldots$

5.8 Conclusion $\ldots \ldots \ldots \ldots \ldots \ldots \ldots \ldots$

6 Conclusions and Future Work $\ldots \ldots \ldots \ldots \ldots \ldots \ldots$

6.1 Major Research Contributions $\ldots \ldots \ldots$. . . . . . . . 71

6.2 Future Work . . . . . . . . . . . . . . . . . . 72 


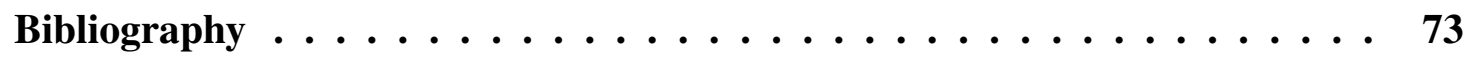




\section{List of Figures}

1.1 Proposed paradigms of Dynamic Spectrum Access (DSA) to overcome underutilization of RF Spectrum . . . . . . . . . . . . 2

2.1 Application of location awareness in cognitive radio networks w.r.t cognition cycle ....................... 7

2.2 Proposed receiver location estimation technique using prior information concerning receiver sensitivity thresholds $\ldots \ldots \ldots 17$

$3.1 \quad$ System setup for overlaid CR network involving primary and CR links . 23

4.1 System Model for location unaware (LUS) and location aware (LAS)

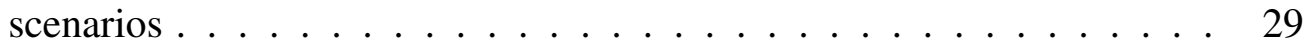

4.2 Impact of increasing system capacity $(\mathrm{M})$ and decreasing detection thresh-

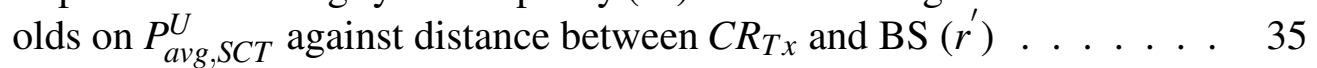

4.3 Impact of increasing system capacity (M) and decreasing detection thresh-

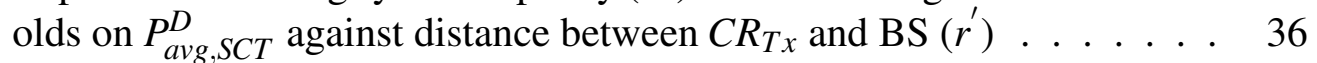

4.4 Impact of decreasing minimum power requirement $\left(P_{\min , M S}\right)$ on $P_{a v g, S C T}^{D}$ against distance between $C R_{T x}$ and $\mathrm{BS}\left(r^{\prime}\right) \ldots \ldots \ldots . \ldots . \ldots 37$

4.5 Impact of decreasing minimum power requirement $\left(P_{\min , B S}\right)$ on $P_{a v g, S C T}^{U}$ against distance between $C R_{T x}$ and $\mathrm{BS}(r) \ldots \ldots \ldots . \ldots 38$

5.1 Introduction of Dummy CR nodes for case where number of primary users is more than number of CR users . . . . . . . . . . . . . . . . . 49

5.2 Introduction of Dummy PU nodes for case where number of CR users is more than number of primary users . . . . . . . . . . . . . 50

5.3 Effect of Density of Users on Average Network Throughput . . . . . . . 55

5.4 Effect of User Density of Number of Admitted Users . . . . . . . . . . 56

$\begin{array}{lll}5.5 & \text { Effect of Distance between Cr pairs on Average Network Throughput }\end{array}$

5.6 Effect on Distance between CR pairs on Number of Admitted Users . . 57

5.7 Effect of Propagation Environment on Average Network Throughput of CR Network . . . . . . . . . . . . . . 58 
5.8 Effect of Propagation Environment on Number of Admitted Users . . . 59

5.9 Effect of Minimum SIR Thresholds on Average Network Throughput. . 60

5.10 Effect of SIR Thresholds on Number of Admitted CR Users . . . . . . . 60

5.11 Effect of PU Activity on Average Network Throughput of the CR Network 61

5.12 Effect of PU Activity on Number of Admitted CR Users . . . . . . . . 62

5.13 Effect of Density of Users on Average CR Network Throughput. . . . . 63

5.14 Effect of Density of Users on Number of Admitted CR Users. . . . . . . 64

5.15 Impact of Location Awareness on CR network throughput for differing levels of separation between CR transmitter and CR receiver. . . . . . . 65

5.16 Impact of Location Awareness on number of admitted CR users for differing levels of separation between CR transmitter and CR receiver . . . 65

5.17 Impact of nature of propagation environment on average CR network Throughput for PLA and FLA. . . . . . . . . . . . . . 66

5.18 Impact of nature of propagation environment on number of admitted CR users for PLA and FLA. . . . . . . . . . . . . . . 66

5.19 Impact of SIR thresholds on CR network throughput for FLA and PLA. 67

5.20 Impact of SIR thresholds on number of admitted CR users for FLA and PLA. ....................... 68

5.21 Impact of PU duty cycle on CR network throughput for FLA, PLA and NLA scenarios. . . . . . . . . . . . . . . . 69

5.22 Impact of PU duty cycle on number of admitted CR users for FLA, PLA and NLA scenarios. . . . . . . . . . . . . . . . . . . . . 69 


\section{List of Tables}

2.1 Receiver Sensitivities of some Wireless Standards . . . . . . . . . . 18 


\section{Acknowledgments}

I would like to express my deepest and sincere gratitude to my supervisor, Dr. Zhi (Gerry) Tian for her encouragement, inspiration, deep insight and invaluable advices, without which this thesis would not have been possible.

I would also like to extend my thanks to the committee members of this thesis, Dr. Philip Merkey and Dr. Chunxiao Chigan. I highly appreciate their precious time and efforts. Their valuable suggestions and comments have helped me improve the quality of this thesis.

I would like to also take this opportunity to sincerely thank my research mates. The presentations, seminars, and discussions during our weekly group meeting have furthered my knowledge in areas of wireless communication and signal processing.

Last but not least, my deepest thanks and kindest love goes to my family for their support and encouragement. 


\begin{abstract}
Dynamic spectrum access (DSA) aims at utilizing spectral opportunities both in time and frequency domains at any given location, which arise due to variations in spectrum usage. Recently, Cognitive radios (CRs) have been proposed as a means of implementing DSA. In this work we focus on the aspect of resource management in overlaid CRNs. We formulate resource allocation strategies for cognitive radio networks (CRNs) as mathematical optimization problems. Specifically, we focus on two key problems in resource management: Sum Rate Maximization and Maximization of Number of Admitted Users. Since both the above mentioned problems are NP hard due to presence of binary assignment variables, we propose novel graph based algorithms to optimally solve these problems. Further, we analyze the impact of location awareness on network performance of CRNs by considering three cases: Full location Aware, Partial location Aware and Non location Aware. Our results clearly show that location awareness has significant impact on performance of overlaid CRNs and leads to increase in spectrum utilization efficiency.
\end{abstract}




\section{Chapter 1}

\section{The Beginning}

\subsection{Introduction}

For today's wireless networks based on fixed spectrum assignment, the outburst in the volume of wireless services and associated traffic, has severely limited the availability of wireless spectrum. However, spectrum occupancy studies in the recent years have shown that the radio frequency (RF) spectrum at any given time and location is either highly underutilized or unutilized most of the times [1]. Given the present nature of static/fixed frequency allocation, where licensees are assigned on a lease basis without considering the actual usage, such unutilized or underutilized spectrum slots are inevitable. The inability of current spectrum allocation strategies to adjust according to fluctuations in spectrum usage, has resulted in inefficient spectrum utilization. This has stimulated various engineering, economic and regulation communities to strive for more efficient spectrum management policies. Among them the Federal Communications Commission has decided to revise the process of spectrum allocation, and consider usage of underutilized/unutilized licensed bands by unlicensed devices.

Therefore, a new concept of spectrum allocation known as Dynamic Spectrum Access (DSA) [2] has been proposed. Unlike the current fixed spectrum access DSA allows users to operate on channels, which are dynamically assigned by taking into account current spectrum usage. The spectrum usage at any given time and location is obtained by continuous monitoring of the RF spectrum (a.k.a spectrum sensing [3]).

The IEEE 802.22 is the first standard that defines the architecture for Wireless Regional Area Networks (WRAN) [4], which operate on unused TV bands between 54 and $864 \mathrm{MHz}$. The prime purpose behind WRANs is to provide broadband connectivity in rural areas by allowing license exempt devices to occupy unused TV bands on a non interfering basis. 


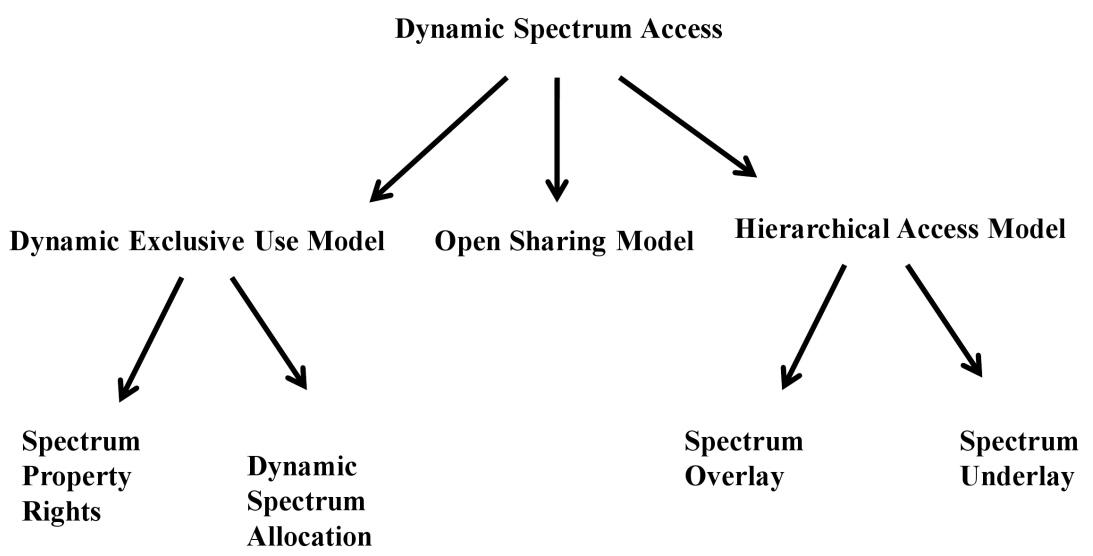

Figure 1.1 - Proposed paradigms of Dynamic Spectrum Access (DSA) to overcome underutilization of RF Spectrum

\subsubsection{Dynamic Spectrum Access (DSA)}

As pointed out earlier, DSA enables users to operate on channels, which are dynamically assigned by taking into account current spectrum occupancy. Several DSA strategies have been proposed in the recent past and can be broadly categorized in three models, as illustrated in Figure 1.1 .

1. Dynamic Exclusive Use Model: This model preserves the basic structure of the current spectrum allocation strategy (fixed), where spectrum is assigned to licensees for exclusive use [2]. Two approaches have been proposed for this model: Spectrum Property Rights [5],[2] and Dynamic Spectrum Allocation [2]. The spectrum property rights approach allows licensees to sell and trade spectrum among themselves based on economy and market[2]. Whereas, the dynamic spectrum allocation relies on exclusive allocation of spectrum to licensees much like today. However, the allocation process is not fixed and occurs at a faster rate as compared to the current spectrum allocation strategies [2].

2. Open Sharing Model: This model proposes open sharing of spectrum among peer users, much like in the ISM band [2].

3. Hierarchical Access Model: This model proposes a hierarchical structure for spectrum access, where primary or licensed users are given topmost priority and secondary users (SUs) are only allowed to operate on common spectrum under strict 
interference constraints [2]. Specifically, two paradigms have been proposed: Spectrum Underlay and Spectrum Overlay.

Since the primary and secondary users operate concurrently on overlapping spectrum, they are bound to cause interference to each other. The spectrum underlay approach imposes severe interference constraints on SU transmissions. SUs transmit only when interference caused by them at PUs is below a threshold, which can be defined either in terms of interference temperature [6] or an acceptable SIR level.

Differing from spectrum underlay, spectrum overlay does not necessarily impose severe constraints on the transmission power of secondary users, but rather on when and where they transmit [2]. Specifically, spectrum underlay is more intrusive as compared to spectrum overlay approach.

\subsubsection{Cognitive Radio Networks}

Cognitive radio networks (CRNs) have the potential to solve the problem of spectrum scarcity by enabling dynamic spectrum access (DSA), which aims at adaptively utilizing spectrum opportunities in space, time and frequency that are offered by temporal and spatial variations of spectrum usage in a multiuser environment. The key feature that distinguishes a CR from an existing wireless device is its capability to sense, be aware of, learn and adapt to its surrounding environment [7].

The bursty nature of PU wireless traffic gives rise to temporal spectrum opportunities i.e., white spaces, which can be used by SUs for communication. Owing to large scale propagation effects such as path loss and shadowing, the strength of wireless signals from PUs degrades upon moving farther, thus enabling SUs to reuse PU bands similar to frequency reuse in cellular networks. The former types of spectrum opportunities are termed as temporal spectrum holes whereas the latter are known as spatial spectrum holes.

Temporal spectrum holes provide prospective opportunities for transmission when a PU is in idle state. Whereas spatial spectrum holes enable CR nodes to reuse primary frequency bands without causing harmful interference to the PUs. However, characterization and efficient utilization of spatial spectrum holes requires location awareness, which comprises of information regarding location of primary and secondary/CR users, knowledge of propagation environment ( $n$ and $\sigma$ ) and network entity parameters (transmission power limits, receiver sensitivity etc.) Location awareness can be acquired either by location sensing or location estimation [8]. Therefore, CRs in the context of DSA can be defined as radios that are aware of their surrounding environment and can adaptively change their transmission parameters based on spectrum availability. 


\subsection{Research Challenges and Motivations}

The erratic variation of spectrum availability over time and location presents an overwhelming challenge for designing different aspects of resource management for CRNs, which include spectrum sensing, spectrum access, admission control, channel allocation and QoS provisioning. Resource management in CRNs aims at optimally utilizing transmission opportunities, subject to given constraints such as no. of users, transmission power limits, receiver sensitivity etc. As discussed before transmission opportunities can either be characterized either as temporal or spatial. The spectrum underlay paradigm has the capability of utilizing both temporal and spatial transmission opportunities, whereas spectrum overlay can only utilize temporal spectrum holes. However, the implementation complexity of underlay paradigm is much more because of the associated preprocessing required for primary user protection. Most of the current work has focused on cognitive radio network (CRN) design based on spectrum overlay. However, a CRN based on the spectrum underlay paradigm is capable of using both spatial and temporal spectrum holes, and will be more spectrally efficient as compared to a CRN based on the overlay model.

But characterization and efficient utilization of spatial spectrum holes requires location awareness, which comprises of prior information regarding location of primary and secondary/CR users, knowledge of propagation environment ( $n$ and $\sigma$ ), network entity parameters (transmission powers, receiver sensitivity etc.). On the other hand acquiring location awareness incurs additional cost in terms of system complexity, power consumption etc. Therefore, there is a need to quantify the performance gains offered by location awareness with reference to the overhead incurred. Also, since there exists a relationship between system complexity and performance, and it is imperative to analyze this relationship and identify scenarios where increase in performance is justified at the cost of increase in system complexity.

\subsection{Research Contributions}

The main contributions of this thesis are listed in the following:

- The impact of incorporating location awareness during the formulation of resource management strategy for single CR user is analyzed, by considering 2 extreme cases: FLA and NLA. Further, impact of system parameters and propagation environment on overlaid CRN performance is examined. In addition, analytical expressions governing the successful coexistence of CRs and PUs are presented.

- Building upon previous results, the analysis is extended to a multi PU and CR user case. Here, the resource management problem is formulated as a mathematical 
optimization problem, with variables denoting transmission power and frequency channel assignments. Specifically, we focus on two key problems in system design: Maximization of Admitted Users and Sum Rate Maximization. In order to contrast and compare effect of degree of location awareness on CRN performance we consider three cases: FLA, PLA and NLA. Since the formulated optimization problems are NP hard, due to presence of binary variables we propose novel graph based algorithms for optimally solving the formulated optimization problems.

- Further, for the PLA scenario we propose a novel technique for Primary Receiver (PR) localization using prior knowledge of receiver sensitivity. The proposed technique can be very useful in estimating location of passive PRs i.e., receivers that are always or mostly listening, such as TV receivers.

- Our Work clearly identifies scenarios where location awareness can prove to be a useful asset, along with scenarios where location awareness does not offer justifiable advantages and thus can be relinquished.

\subsection{Outline of the Thesis}

The remainder of the thesis is organized as follows. Chapter 2 provided the required background and literature review. Chapter 3 introduces the system model adopted for this work. In Chapter 4, we analyze the impact of location awareness on network performance for a single CR user case. Motivated by results obtained in chapter 4, we consider a much more pragmatic scenario involving multiple CR and PU links in chapter 5. Finally, chapter 6 gives concluding remarks of this thesis and outlines possible future work. 


\section{Chapter 2}

\section{Background and Literature Review}

The successful implementation of CRNs depends on two critical processes: spectrum sensing and spectrum access. A great detail of work has been done in both the areas. However, there has been no firm decision yet regarding the means of carrying out these processes. All the proposed methods so far have their set of advantages and disadvantages. This chapter provides a literature review on the related work done in the areas of spectrum access and spectrum sensing. Further, the concept of location awareness is introduced, along with a means of classifying different CR systems based on the associated degree of location awareness. Finally, prospective methods required to acquire the needed location awareness are outlined.

\subsection{Cognition Cycle}

According to [9], "Cognitive Radio is defined as an intelligent wireless communication system that is aware of its surrounding environment (i.e., outside world), and uses the methodology of understanding - by - building to learn from the environment and adapt its internal states to statistical variations in incoming $R F$ stimuli by making corresponding changes in certain operating parameters (e.g., transmit-power, carrier frequency, and modulation strategy) in real time, with two primary objectives in mind:

\section{Highly reliable communications whenever and wherever needed.}

\section{Efficient utilization of radio spectrum."}

Some of the key words that standout in this definition are: awareness, intelligence, learning, adaptivity, reliability, and efficiency. The inter relationship between these characteristics is efficiently depicted by the cognition cycle [7],[9]. The three key cognitive tasks to be performed by CR devices are [9]:Spectrum Sensing, Channel Identification 


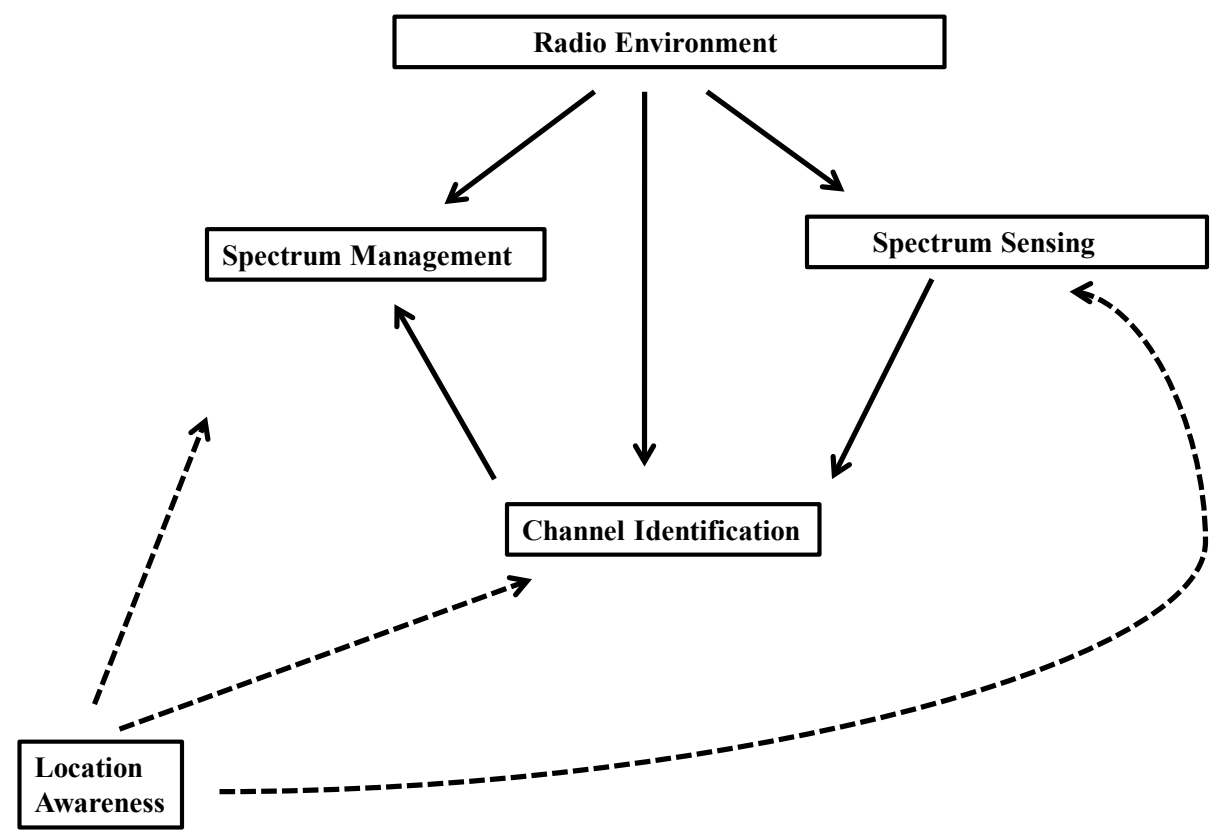

Figure 2.1 - Application of location awareness in cognitive radio networks w.r.t cognition cycle

and Resource Management. These are described in detail in the following sections. Location Awareness in CRs, which is the main focus of this work has found application in all the key aspects of CRs:Spectrum Sensing [10], Channel Identification [11] and Spectrum Management.

\subsubsection{Spectrum Sensing}

Before a CR changes its communication parameters to reuse a PU band, it has to monitor spectrum usage in its vicinity. This stage involves detection of spectrum holes i.e., prospective transmission opportunities. Spectrum holes can be detected by a process known as spectrum sensing, which aims at detecting presence of primary users. Sev- 
eral spectrum sensing strategies have been proposed so far. Some of them are briefly described below:

1. Energy Detector Based Sensing: Energy detector based spectrum sensing is the simplest mode of spectrum monitoring because of its low implementation cost and low computational complexity [12]. It is the most generic form of spectrum sensing as compared to its peers, as receivers need not be aware of the nature of primary user signals. The primary user detection relies on comparing the output of a energy detector to a threshold value, whose value depends on noise floor [13]. However, the energy detector based approach is faced with numerous challenges such as selection of detection threshold, inability to differentiate between primary user transmissions and noise, poor performance in low (Signal to Noise Ratio) SNR environments and inability to monitor wide bandwidth [3].

2. Waveform Based Sensing: Most of the wireless signals are embedded with known patterns, which are used for assisting synchronization or other purposes. Such patterns may include preambles, pilot symbols, spreading sequences etc. In the presence of such known patterns, sensing can be performed by correlating the received signal with a copy of itself. This method is only applicable to systems with known signal patterns and is termed as waveform based sensing [14].

3. Cyclostationarity Based Sensing: This method relies on detection of primary users by exploiting the cyclostationarity features of the received signal [15]. Cyclostationarity features are caused by the periodicity in the signal or in its statistics such as mean and variance. This method uses the cyclic correlation function instead of the power spectral density for detection of primary signals. This method is highly robust to noise owing to the wide sense stationarity property of noise [16].

4. Matched Filtering: Matched filtering is the optimum method for spectrum sensing when the transmitted signal is known [17]. Key advantages of matched filtering include fast computation as compared to its peers. However, the required number of samples grows as $O(1 / S N R)$ for a target probability of false alarm, at low SNRs [3].

However, spectrum sensing is faced with a number of challenges such as:

1. Nature of Propagation Environment: Large scale propagation effects such as shadowing and multi path fading adversely affect the process of detection of PUs. Such inaccurate detections may lead to harmful interference at PUs, due to resulting CR transmissions. Therefore, efficiency of spectrum sensing depends significantly on the nature of environment for scenarios where the propagation conditions are 
harsh. Several methods such as cooperative spectrum sensing have been proposed to overcome this problem, where the decision concerning the presence of a PUs in not solely based on a single CR users, but instead relies on multiple sensors [18].

2. Hardware Challenges: Spectrum sensing involves monitoring spectrum sensing over a wide bandwidth. However, according to the Nyquist theorem as bandwidth increases the sampling rate too increases. The increase in requirement of high sampling rates imposes a hardware challenge, as current Analog to Digital Converters (ADCs) are unable to operate efficiently at such high frequencies. Recently, Compressed sensing has emerged as a feasible solution for reducing the sampling rate requirement of wide band spectrum monitoring [19].

3. Sensing Frequency and Duration: In the Opportunistic Spectrum Access (OSA) model, the PUs can claim their band anytime. At such instances the SUs operating within that band are required to detect the presence of PUs immediately and vacate the band. Another critical issue in spectrum sensing is the sensing rate, i.e., should the CRs sense the required bands continuously and at which rate? The detection limit time and sensing rate impose challenges during CR system design.

Based on the results of spectrum sensing the obtained spectrum holes can be categorized in accordance to their occupancy as: Highly Utilized (Black Spaces), Partially Utilized (Grey Spaces) and Utilized (White Spaces) [9]. Recently, it has been shown that incorporating location awareness in spectrum sensing yields to superior performance [20]. Authors in [20] utilize location awareness, which consists of prior information concerning geographical location of primary users, to derive a closed-form optimal spectrum sensing threshold to maximize a cognitive network weighted sum capacity.

\subsubsection{Channel Identification}

Computation of channel capacity of a SU link requires knowledge of channel state information (CSI). The required CSI consists of channel information concerning channel from SU transmitter to PR and channel from SU transmitter to SU receiver. Acquisition of CSI differs for both the above mentioned channels.

\subsubsection{Resource Management/Spectrum Management}

Frequency and transmission power are the key resources in any wireless communication system. Choice of frequency channels and transmission power directly impacts network performance, therefore they need to be wisely chosen given the system constraints.

Based on the information collected in the previous stages, here a resource management strategy is formulated, which responds to CR requests and assigns transmit power, 
frequency channels etc to CR users. Development of resource management strategies based on different levels of location awareness is the key theme of this work and will be discussed in more detail in the following chapters.

\subsection{Location Awareness}

According to [21], the awareness capability of cognitive radio embodies awareness with respect to the transmitted waveform, RF spectrum, communication network, geography, locally available services, user needs, language, situation and security policy.

Awareness or specifically location awareness can be regarded as a three dimensional feature. The three dimensions are defined by location in terms of time, frequency and geographical location. A CR is expected to take decisions (change its transmission parameters) based on the RF stimuli it receives via its capability to acquire awareness of the environment, in which it operates. Therefore, the degree of awareness of a CR is bound to affect its resource management strategy, which directly affects spectrum utilization. The three dimensions of location awareness can be described in detail as:

1. Time - Since usage of PU bands are non uniform over time, therefore the CR nodes should be able to utilize this information during the formulation of resource management and spectrum sensing. Specifically, the this can affect sensing duration and spectrum sensing frequency. A RF band at during its peak utilization is bound to offer less transmission opportunities to CRs, as compared to times where the utilization is much less. A temporal aware CRN can thus adjust its sensing duration and frequency in accordance to the band usage, thereby saving battery life. Such temporal awareness can be acquired by monitoring of RF spectrum and devising prediction models based on techniques such as Neural Networks, capable of predicting use of RF Bands.

2. Frequency - The RF spectrum spans from $\mathrm{X} \mathrm{Hz}$ to $\mathrm{Y} \mathrm{Hz}$, where each band has a different set of rules concerning system parameters such as maximum allowed transmission power and receiver sensitivity, along with different propagation characteristics. Thus, in order to increase spectrum utilization for a particular band the operating CR nodes should be aware of its characteristics. Adoption of a common access strategy for all PU bands may lead to sub-optimal performance.

3. Space/geographical Location - The PU network consists of transmitters and receivers. For overlay CRs, it is imperative that interference caused by a CR node should be below the interference threshold of the primary receivers at all times. Geographical location becomes highly important in this regard as it can be used to predict received power at primary receivers, given a signal propagation model. 
However, in order to acquire location awareness we need to incur an additional cost in terms of system complexity. The system should be able to either estimate parameters of interest (locations, channel parameters etc) and process them independently, or acquire them via cooperation with the primary user network. In both cases an additional cost either due to system complexity or additional overhead needs to be incurred. Such a cost is only justified when there is significant increase in performance (increase in network throughput) as a result of its bearing.

Therefore, there is a need to quantify the performance gains offered by location awareness, with reference to the overhead incurred. We consider three cases, differing in the amount of location awareness, in this regard:

1. Full Location Aware (FLA)

2. Partial Location aware(PLA)

3. No Location Awareness (NLA)

Each of these cases will be discussed with regard to:

1. Required Information/Assumptions - Here we define the information available to CR network for formulating its resource management strategy.

2. Degree of Location awareness - Here we quantify the degree of location awareness of the CR system, based on the available information.

3. Benefits- Here we discuss the potential benefits of the system, given the available information.

4. Concerns- Here we discuss the potential concerns and road blocks for the given system.

5. Prospective Information Acquisition Techniques- Here we outline methods that can be used to acquire the needed information for the given scenario (FLA/PLA/NLA).

\subsubsection{Full Location Aware (FLA)}

For the full location aware scenario we assume to have the following prior information concerning network entities:

1. Available Information:

(a) Information concerning geographical location of primary transmitters.

(b) Information concerning geographical location of primary receivers. 
(c) Information concerning propagation environment parameters (path loss exponent, shadowing variance).

(d) Prior information concerning spectrum occupancy by all primary users i.e., the CR network knows which PU uses which band.

\section{Degree of location awareness- Full}

This scenario will be considered as the bench mark, and performance of all other scenarios will be compared with FLA.

3. Benefits:

(a) Can utilize both temporal and spatial transmission opportunities with equal efficiency. Given the FLA scenario, a CR network can exploit both temporal and spatial spectrum opportunities. Temporal transmission opportunities arise when the primary user is idle or in OFF state. Whereas spatial opportunities enable CR nodes to reuse primary frequency bands, without causing harmful interference to primary receivers. Efficient and harmless utilization of spatial spectrum opportunities relies on reliable and robust prediction of received signal power due to $\mathrm{CR}$ nodes, at primary receivers. If prior information regarding geographical location of primary receivers (PRs) is known, then inter distances between CR nodes and PRs can be estimated, which can be used during the formulation of the resource management problem. However, if no prior information is available regarding geographical location of PRs, the CR network is forced to adopt a far more conservative approach and does not utilize spatial spectrum opportunities.

(b) Can help guarantee quality of service to primary users (minimization of outage probability). As estimates of inter distances between CR nodes and PRs can be obtained given the available information, the primary receivers can be guaranteed a minimum level of QoS defined by the outage probability of the CR network i.e., probability that the received interference power at a primary receiver exceeds a predefined threshold (Interference Temperature).

(c) Will help achieve better spectrum utilization in scenarios where primary receivers are always/mostly listening. In cases where communication occurs in down link mode most of the time, the chances of localizing primary receivers using techniques such as TOA, AOA are much less. Therefore, only temporal spectrum opportunities can be exploited in such scenarios. However, given the information regarding geographical location of primary receivers, both temporal and spatial spectrum opportunities can be exploited, thereby increasing spectrum utilization. 
(d) Will prove beneficial in bands having high duty cycle. Intuitively, in frequency bands with a high duty cycle i.e. primary transmitter is transmitting with a high probability, the only spectrum opportunities available to location unaware CR nodes are the temporal holes. However, since the primary transmitter is transmitting most of the time, the possibility of accommodating CR nodes is very less. But by incorporating location awareness in the CR nodes, additional $\mathrm{CR}$ nodes can be accommodated using spatial transmission opportunities.

\section{Concerns}

(a) Will require great deal of overhead information. Due to the large amount of information required to devise the resource management strategy, network overhead can prove to be a road block for this scenario.

\section{Prospective Information Acquisition Techniques}

Here we summarize techniques that can be used to acquire the needed information for this scenario.

(a) Prior information concerning location of primary transmitters can be acquired in a variety of ways:-

i. Geo location databases - According to the Geo location - Database scheme, licensed users (i.e. TV transmitters) are equipped with location estimation and/or sensing device to estimate their current location information [3]. Licensed users provide their spectrum and location information to the FCC central database. Hence, information concerning location of primary transmitters and their spectrum occupancy can be acquired by accessing the FCC database.

ii. Cooperation with primary network - The CR network and PN may share information concerning location of their respective transmitters. Authors in [22] propose a two phase mixed distributed/centralized control algorithms that rely on cooperating with the $\mathrm{PN}$, to maximize coverage of an overlaid CRN while protecting primary users. A new cooperation protocol is introduced in [23], which allows active cooperation between primary users and CR users. Differing from other works, this protocol guarantees continuous operation to CR users, which is attained by obtaining spectrum from the PU in lieu of assistance in relaying PU data [23]. A CRN based on system models similar to [22],[23] can acquire information regarding primary transmitters from PRN via cooperation. 
iii. RSS based methods- Active primary transmitters within an area can be localized cooperatively by CR nodes, using RSS based methods. Depending on whether transmit power of transmitters is known a priori methods outlined in [24] can be used. Authors in [24] introduce a robust technique for estimating the position and transmission power of a primary transmitter. Further, they introduce a constrained optimization method to solve the formulated estimation problem and prove its superiority over the conventional least square method.

A maximum likelihood (ML) algorithm along with associated performance bounds is introduced in [25], for estimating a primary transmitter's position, orientation, beam width, transmit power and path loss exponent of propagation environment using RSS.

(b) Prior information concerning location of primary receivers.

This is perhaps one of the most challenging problems in the deployment of CRNs. Some possible means of acquiring this information include:

i. Cooperation with primary network If system setup is similar to the ones considered in [22], [23], where there exists active cooperation between PUs and CRs, this information can be readily obtained. The PN can share information concerning location of PRs in lieu of additional revenue from CRN or CRs can assist PN by relaying its data to PRs [23]. Authors in [26] propose a PR detection scheme, which relies on PRs communicating with SUs to provide information such as location, frequency occupancy via a semaphore architecture. Here "semaphore" refers to the signal transmitted from the PR to SUs.

ii. Detection of Local Oscillator Leakage Power. In general, primary receivers emit the local oscillator (LO) lakage power from its RF front end in during the process of reception of data from the primary transmitter. The authors in [27] propose a CR architecture consisting of sensors, capable of detecting LO leakage. These sensors then communicate with the CR devices using predefined channels to deliver information concerning spectrum occupancy. Since location of these sensors can be assumed to be known, they can be used geographical information of location of PRs. A detailed performance analysis in terms of probabilities of false alarm and mis detection, is presented in [28].

iii. Deploying sensors which localize PU receivers, when they transmit (Uplink). This method utilizes the fact, that most of the communication that takes place in wireless networks such as GSM, WLAN is a bidirectional. This gives the CR nodes an opportunity to localize PRs, 
when they transmit (uplink). For localization of PRs CR nodes may either use RSS techniques or TOA, AOA etc.

(c) Prior information concerning propagation:

Information concerning propagation environment consists of knowledge regarding path loss exponent $(n)$.

Estimation of path loss exponent based on RSS can be acquired using any one of the following means:

i. Radio Environment Maps

Radio Environment Maps (REMs) refer to an integrated database consisting of multi domain information, which can help CR nodes optimize their actions [29]. REMs can provide information concerning geography of environment, services and networks available within an area, regulation policies, activity profiles of users and prior experience [29]. Such REMs can be built by CR nodes by collecting information over a period of time and this information can be made available to CR nodes either in a centralized or distributed manner.

ii. Environment Characterization via CRs

It is possible for CR nodes present within an area to jointly characterize the propagation environment by processing of collecting RSS measurements. Authors in [25] propose a framework based on maximum likelihood, to jointly estimate a primary transmitter,s location, orientation, beam width, transmit power along with the path loss exponent of the environment. A error back propagation based method for estimating $n$ in presented in [30].

(d) Information concerning characteristic spectrum occupancy

This information can be gathered in a variety of ways, depending on the system design

i. - Cooperative Primary Network Cooperation between CRN and PN can be used to acquire this information using the Semaphore based framework [26]. Given this framework the PRs present within the area transmit a signal known as "semaphores" to CR users, informing them about their spectrum occupancy. Combing this information with the location information, a database with PRs and their respective frequency occupancy status can be developed.

ii. Non Cooperative Primary Network In case of a non cooperative PN knowledge concerning the Local Oscillator Leakage Power (LOLP) can be used to acquire this information. The local oscillator leakage power from a receiver lies within the interval $[C O I, C O I+41] \mathrm{Hz}$, where $C O I$ 
refers to the bandwidth of the channel of interest [27]. Therefore, detection of a PR via detection of LOLP also gives information regarding its spectrum occupancy.

iii. Combining location information with Spectrum Sensing

Most wireless standards clearly define minimum receiver standards using parameters such as receiver sensitivity, inter modulation characteristics and interference blocking etc. Receiver sensitivity is defined as the weakest RF signal power that can be processed to develop a minimum signal-to-noise ratio for achieving a required error rate, defined by either Bit Error Rate (BER) or Frame Error Rate (FER) [31].

By combining capability of spectrum sensing and prior knowledge of location of PRs, we can attain information regarding characteristic spectrum occupancy of PRs as described. In order to guarantee the minimum the RF signal at the receivers, transmitters usually employ some sort of power control mechanism, by which they control their transmission power in accordance to channel conditions and distance. The transmission power for a receiver located farther from the transmitter would be more as compared to a receiver located nearer to the transmitter.

By placing sensors capable of wide band spectrum sensing, at PR locations we can identify their characteristic frequency occupancy, as will be described in detail in the preceding chapters.

\subsubsection{Proposed Method of PR Location Estimation}

The successful implementation of overlaid CR networks lies in their capability to control their transmit power, and thus avoid causing interference at PRs. However, quantification of interference power perceived by PRs requires the CRN to have knowledge regarding location of PRs present within the area. However, acquisition of such information increases system complexity, as methods described in impose additional cost. Here we propose a novel method of estimating PR location using system parameters which are clearly defined by regulatory authorities such as FCC. Specifically, we use the knowledge of receiver sensitivity and location of primary transmitter, both of which can be obtained with ease, to estimate location region of PRs.

Consider an CR network coexisting with a PN, which consists of a central base station (BS) and $k$ primary receivers. The CR network comprises of CR nodes distributed within the area as shown in Fig. 2.2

The PN communicates with the PRs present within its coverage using characteristic frequency bands such that the $i^{t h} \mathrm{PR}\left(P R_{i}\right)$ is assigned the $i^{t h}$ band, and adopts a adaptive transmit power control (ATPC) mechanism to control its transmission power 


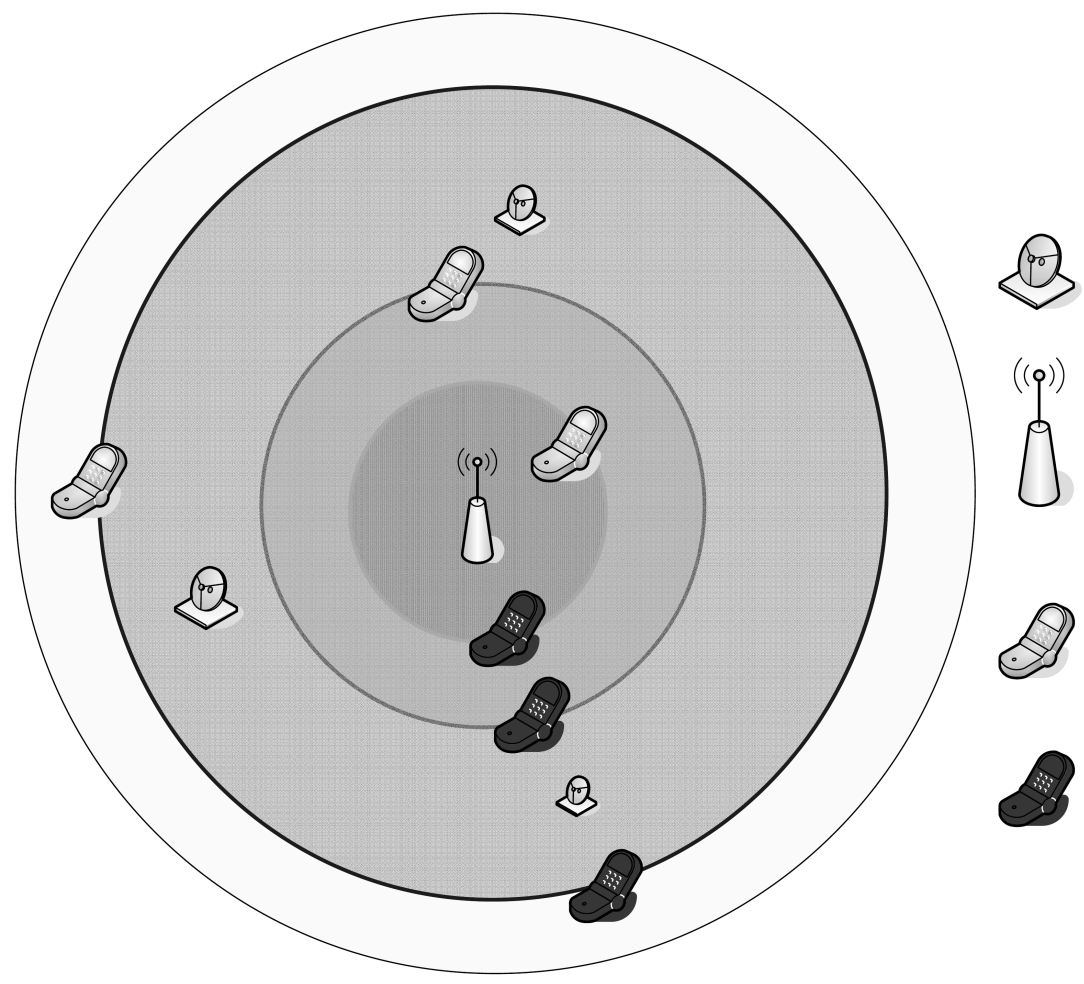

Sensors

Primary Transmitter (BS)

\section{Primary}

Receiver

(FLA)

Primary Receiver

(PLA)

Figure 2.2 - Proposed receiver location estimation technique using prior information concerning receiver sensitivity thresholds

in accordance to its distance from the intended receiver. The ATPC aims mechanism at guaranteeing a min signal power of $P_{\text {Min }}$ at all PRs for their characteristic bands.

This can be mathematically expressed as:

$$
P_{t x}^{i}=P_{\text {Min }}-10 n \log _{10}\left(d_{i}\right) \forall \quad i \quad \varepsilon \quad k
$$

where $d_{i}$ represents the distance of $P R_{i}$ from the BS.

The sensors present in the area monitor all the $i$ bands by keeping a track of the received powers. For a particular band $k, n$ sensors are chosen out of the $N$ sensors, such that the received power at these $n$ sensors is closest to $P_{M i n}$. The reason for choosing $n$ sensors out of $N$ is to combat the effects of shadowing and fading.

We assume that all sensors know their location in space using techniques as GPS and have knowledge of the primary transmitter's location. The chosen $n$ sensors estimate their distance from the PTX using their prior information.

$$
d_{k}^{\prime}=\sqrt{\left(X_{j}-X_{P T X}\right)^{2}+\left(\left(Y_{j}-Y_{P T X}\right)^{2}\right)} \quad \forall \quad k \quad \varepsilon \quad n
$$


Table 2.1

Receiver Sensitivities of some Wireless Standards

\begin{tabular}{ccc}
\hline \hline S.No & Wireless Standard & Required Receiver Sensitivity $(\mathrm{dBm})$ \\
\hline 1 & GSM & -102 \\
2 & CDMA & -104 \\
3 & GPRS & $-102 /-99$ \\
4 & EDGE & -98 \\
5 & WCDMA & -106.7 \\
6 & $802.11 \mathrm{a}$ & {$[-74,-89]$} \\
7 & $802.11 \mathrm{~g}$ & {$[-73,90]$} \\
8 & $802.11 \mathrm{~b}$ & {$[-85,-95]$} \\
9 & DTV & -84
\end{tabular}

Where $\left(\left(X_{j},\left(Y_{j}\right)\right.\right.$ and $\left(X_{P T X}, Y_{P T X}\right)$ represent the position of the the $j^{t h}$ sensor and primary transmitter respectively.

The circular region centered at $\left(X_{P T X}, Y_{P T X}\right)$ and radius equal to $d_{k}^{\prime}$, represents the possible location of $P R_{k}$. Similarly concentric circular regions can be obtained for the other PRs as shown in fig. 2.2.

\subsubsection{Partial Location aware CRNs}

Required Information:

1. Information concerning geographical location of primary transmitters. This can be obtained using similar methods, as for FLA CRs.

2. Information concerning receiver sensitivity.

Most wireless standards define minimum desired receiver performance in terms of receiver sensitivity. Table 2.1 lists minimum desired receiver sensitivities for some common wireless standards.

The PLA CR network utilizes the proposed method to estimate circular regions for each PR present within the area. However, given the information it is impossible to localize the PR with utmost accuracy. Therefore, the PLA CR adopts a conservative approach and assumes the PR to be located at the nearest point on the circular region for every primary frequency band as shown in fig 2.2 .

3. Information concerning propagation environment parameters

Information concerning propagation environment can be obtained in the manner similar to FLA CRs. 
4. Information concerning spectrum occupancy by all primary users

The PLA CR network utilizes the method proposed in section 2.2.2 to estimate the location of the PRs. This method relies on the prior knowledge of receiver sensitivity, location of primary transmitter and wide band spectrum monitoring sensors. Each circular region corresponds to a characteristic frequency.

Degree of location awareness- Partial

Benefits

1. Will be able to utilize both temporal and spatial opportunities. Like the FLA CRs, PLA CRs will be able to utilize both spatial and temporal opportunities for transmission. However, due to the conservative approach of PLA CRs towards PR location, it will be later seen that the performance of PLA CRs is less as compared to FLA CRs in terms of average network throughput and no. of supported users.

2. Will be able to provide QoS to primary users.

Like the FLA CRs, PLA CRs will utilize spatial transmission opportunities only when QoS is guaranteed to the PRs i.e., SIR threshold for PRs is not exceeded. Thus, protection of PRs can be guaranteed.

\section{Concerns}

1. Will utilize temporal opportunities with more efficiency as compared to spatial opportunities.

Due to lack of precise information of PRs, CRs are forced to adopt a more conservative approach as compared to FLA and therefore are not able to utilize spatial spectrum holes as efficiently as FLA CRs.

2. Performance is dependent on accuracy of sensors and their layout (density, a denser sensor network will result in better accuracy regarding location of primary receivers).

The PLA CRs as described in section utilize the information regarding receiver sensitivity of PRs along with information from sensors, capable of performing wide band spectrum sensing to obtain prospective regions for PR location. Accuracy of obtaining such regions is directly related to accuracy of sensors. monitoring the RF environment. Therefore, performance of PLA CRs depends on accuracy of the sensors, comprising the sensor network.

3. Will increase complexity of nodes involved as they will be required to perform location sensing. 
It may possible to allow the $\mathrm{CR}$ nodes present in the area perform wide band spectrum monitoring, instead of deploying a separate sensor network. However, this will require embedding of more computational power as compared to FLA CRs, thereby increasing node complexity and power consumption.

Feasibility of Information acquisition

1. Location of PU transmitters - Locations of the primary transmitters can be estimated using means described in section 2.2.1.

2. Knowledge of receiver sensitivity - Minimum receiver sensitivity thresholds are predefined for most of the current wireless standards 2.1.

3. Knowledge regarding propagation environment - Information concerning RF propagation conditions can be obtained using means outlined in section 2.2.1.

\subsubsection{Non location Aware CRNs}

Available Information

1. Information concerning propagation environment parameters (path loss exponent).

2. Prior information concerning spectrum occupancy by all primary users i.e., has knowledge regarding temporal spectrum opportunities only.

Benefits

1. Will be able to use temporal spectrum opportunities efficiently.

Temporal spectrum opportunities arise when the PU is in "Idle/OFF" state. Given the knowledge of temporal spectrum holes, NLA CRs will be able to utilize them efficiently without worrying about PU protection.

2. Simplest in complexity.

Since the NLA CRs are capable of operating independently i.e., without any cooperation with PUs, they are simplest in complexity as compared to FLA and PLA CRs.

\section{Concerns}

(a) Will only utilize temporal spectrum opportunities.

(b) System performance is dependent on spectrum sensing, Spectrum sensing is responsible for providing NLA CRs with knowledge regarding opportunities for transmission. Thus, system performance of NLA CRs is sensitive to sensing errors. 


\section{Feasibility of Information Acquisition}

(a) Propagation Environment Characteristics

Can be obtained using methods described in section 2.2.1.

(b) Spectrum Occupancy

Can be obtained using spectrum sensing methods outlined in section 2.2.2. 


\section{Chapter 3}

\section{System Model}

In cognitive radio networks, there are two types of users: primary and secondary. The primary users are part of the primary network, which consists of a primary base station and a number primary users, distributed uniformly within the coverage of the base station. Each of the primary users possess a license to exclusively use one frequency band for either downlink or uplink transmission. The primary network setup adopted in this work resembles any standard wireless infrastructure network, such as cellular, TV transmission etc. Further, the cognitive radio network comprises of secondary users equipped with frequency agile devices knowns as CRs. The SUs aim to opportunistically reuse primary user frequency bands to communicate with their intended single hop receivers, subject to interference constraints at primary users. Figure 3.1 shows a typical CR network overlaid on a primary network. The PUs are legacy systems providing communication range with relatively large coverage range (of the order of $0.5 \mathrm{kms}$ for Cellular networks or few miles for TV broadcasting). On the other hand, the communication range of the SUs is small compared to that of PUs. Therefore, the SUs are present in communicating range of each other and form a single hop network covering a small area; however CRs can cover a larger area by forming by forming a multi hop ad hoc network. This work precisely deals with single hop CR networks.

\subsection{Primary Network Model}

Regardless of the types of the PUs, the primary networks are considered to operate on $N$ non overlapping frequency bands, such that each primary users is assigned exclusively a single band for either downlink or uplink communication. Each of these $N$ channels can be modeled at any time as either $O N$ or $O F F$. The duty cycle of each $N$ primary channel can be expressed as: 


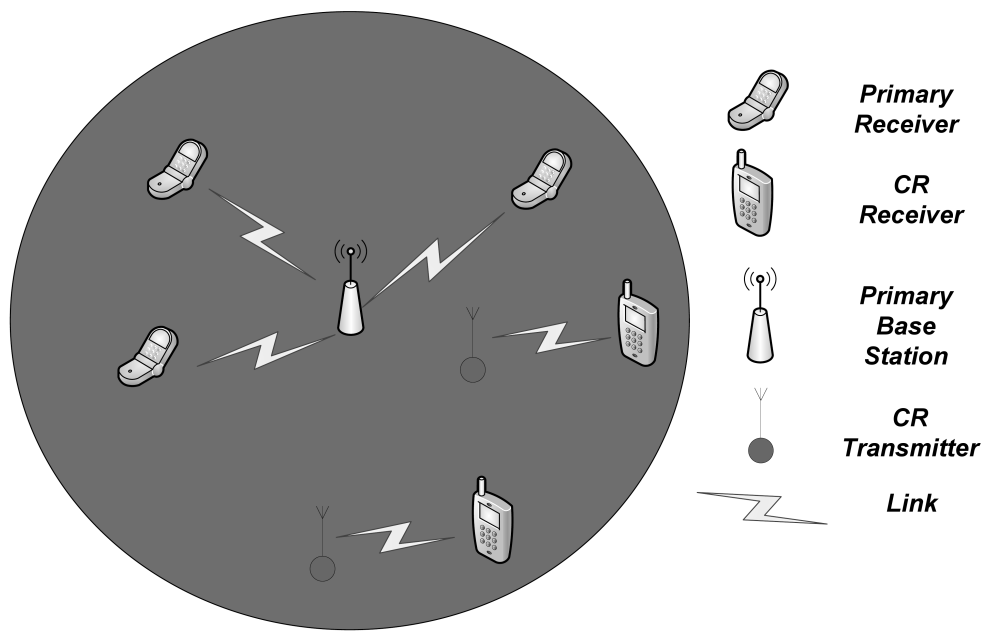

Figure 3.1 - System setup for overlaid CR network involving primary and CR links

$$
\text { DutyCycle }=t_{\text {ON }} / t_{\text {ON }}+t_{\text {OFF }}
$$

Where $t_{O N}$ denotes the duration of time during which the PU is active, and $t_{O F F}$ denotes the time duration during which the PU is inactive.

\subsection{Secondary Network Model}

The network architecture of CRNs can either be centralized or distributed. In a centralized setup, a base station is responsible for monitoring the entire CR network and performs critical functions such as frequency channel assignment, transmit power allocation for CR users. Also, in centralized mode all CR nodes can communicate with the BS using predefined control channels to acquire control information.

But as the scale of the network increases, it becomes infeasible to acquire global information due to the associated overhead. This motivates us to have a distributed setting where CR pairs form small clusters, with critical network functions being carried out by a mutually chosen cluster head. The cluster head in this case devises network strategies based on local information i.e., information gathered from members of a particular group. Such a distributed architecture may lead to suboptimal performance, but nevertheless it reduces network overhead and also results in conservation of power. 
For the current work, we assume a centralized architecture where a CR base station $\left(B S_{C R}\right)$ is responsible for formulating network strategies. All SUs present in the network communicate with $\left(B S_{C R}\right)$ using predefined control channels. 


\section{Chapter 4}

\section{Resource Management in CRNs - Single CR User}

In the emerging spectrum sharing paradigm, cognitive radio (CR) nodes seek spectrum opportunities that can be characterized both in temporal and spatial domains. Temporal spectrum holes provide us with prospective opportunities for transmission when a primary user (PU) is in idle state. Whereas spatial spectrum holes enable CRs nodes to coexist on occupied primary frequency bands without causing harmful interference to the PUs. A system capable of using both spatial and temporal spectrum holes is bound to increase system performance significantly. However spatial spectrum holes require either location sensing or location estimation i.e., localization of primary and secondary users (SUs), and this cost has to be balanced with performance gains offered. To understand the tradeoffs, this work aims to evaluate performance of location aware cognitive radio networks in scenarios where CR users and PUs operate on overlapping frequency bands. This chapter focuses on the paradigm of location assisted dynamic spectrum management for scenarios where primary and secondary users operate on overlapping frequency bands. Using the spectrum utilization efficiency as performance metric, we analyze the performance gain offered by location awareness with respect to key system parameters of legacy networks and wireless channel parameters.

The goal is to evaluate the feasibility of coexistence of CR users and PUs in spatial domain. The concept of probability of successful concurrent transmission $\left(P_{S C T}\right)$ is introduced in [32], but its evaluation hinges on some restrictive assumptions on the system setup, such as single channel availability, constant and same transmission power for both the CR users and PUs.

In this paper we build up on the notion of $\left(P_{S C T}\right)$ and extend it to a much more realistic case. In lieu of the single-channel case in [32], we consider the multi-channel, multi-user case, in which PUs operate on orthogonal channels. In addition, PUs are equipped with adaptive transmit power control (ATPC), which enables them to adjust 
their transmission power in accordance to their distance from the intended receiver. We investigate the relationship between performance of overlaid CR ad hoc networks with respect to wireless channel parameters (path loss and shadowing) and system parameters (system capacity, detection thresholds and transmit power control). In addition, we provide analytical expressions for $P_{S C T}$ and average probability of successful concurrent transmission $\left(P_{\text {avg,SCT }}\right)$. Our analysis also sheds light on the location sensing and estimation requirements for such overlaid cognitive radio networks, which provides useful guideline for system designers to trade off the benefits and costs of location aware networking.

\subsection{System Setup and Problem Statement}

Consider a primary network (PN) consisting of a single base station (BS) and $M$ active mobile stations (MSs a.k.a PUs) uniformly distributed within its coverage $R_{B S}$. Further, a CR ad hoc network comprising of $N$ CR nodes uniformly distributed within a radius $R$, with BS at its center, coexists with the given PN. Secondary/CR nodes present in the area can lie either outside or inside the coverage of the primary BS. Each of the $M$ PUs/MSs present is allocated a distinct frequency band for communication with its BS (in uplink) and vice versa, and are assumed to be stationary.

In the current setting CR nodes concurrently seek to form single hop links with their intended receiver, by reusing one of the $M$ PU bands subject to constraints on interference caused to PUs. Such a single hop link $\left(C R_{T x}, C R_{R x}\right)$, consists of a CR transmitter $\left(C R_{T x}\right)$ located at a distance $r^{\prime}$ from the BS such that $\left(0<r^{\prime} \leq R\right)$ and, a CR receiver $\left(C R_{R x}\right)$. We assume that $\mathrm{CR}$ nodes are aware of the location of their neighboring $\mathrm{CR}$ nodes, which lie within one hop distance from them. Each of these CR peer-to-peer connections uses a distinct PU band, so that interference caused within the CR ad hoc network can be ignored.

Let $\mathrm{SIR}_{I}$ and $\mathrm{SIR}_{A}$ denote the received signal to interference ratios (SIR) for the CR ad hoc and primary network respectively. The essential condition for both a pair of CR nodes and, a primary receiver and transmitter pair to operate successfully on a common frequency band can be expressed as[32]:

$$
\left(\operatorname{SIR}_{I}>\operatorname{SIR}_{I, T}\right) \cap\left(\operatorname{SIR}_{A}>\operatorname{SIR}_{A, T}\right)
$$

where $\mathrm{SIR}_{I}$ and $\mathrm{SIR}_{A}$ represent the received $\mathrm{SIR}$ at $\mathrm{CR}$ receivers and primary receivers respectively. The quantities $\mathrm{SIR}_{I, T}$ and $\mathrm{SIR}_{A, T}$ denote the minimum SIR requirements for the PN and CR respectively. The minimum SIR requirement for the PN $\left(\mathrm{SIR}_{I, T}\right)$ dictates the amount of interference that can be tolerated by PUs, whereas $\mathrm{SIR}_{I, A}$ represents the minimum rate requirement of $\mathrm{CR}$ users. However, in order to estimate 
$\mathrm{SIR}_{I}$ at the PUs the CR nodes need to be aware of the locations of the PU transmitters and receivers.

But embedding location awareness in overlaid $\mathrm{CR}$ ad hoc networks increases system complexity, and is justified if the resultant gains (increase in system spectrum utilization) are significant. To observe the impact of incorporating location awareness in CR ad hoc networks, we determine the performance gap between two scenarios.

- Location Aware Scenario $(L A S)$ - CR nodes are aware of the location of PU transmitters and receivers.

- Location Unaware Scenario (LUS) - CR nodes are aware of the location of the PU transmitters only.

Location information concerning PN can be collected via methods discussed in chapter 2.

In this paper, the adopted figure of merit is probability of successful concurrent transmission $\left(P_{S C T}\right)$, which represents the probability of coexistence of a PU and a CR link on a common frequency band [32]:

$$
P_{S C T}=P\left(\left(\operatorname{SIR}_{I}>\operatorname{SIR}_{I, T}\right) \cap\left(\operatorname{SIR}_{A}>\operatorname{SIR}_{A, T}\right)\right)
$$

Notice that a CR decides to transmit only when it confirms that 4.1 is satisfied. Assuming perfect utilization of temporal spectrum opportunities, the probability of opportunistic transmission $\left(P_{O T}\right)$ can be expressed as:

$$
P_{O T}=P_{S C T} \cdot \operatorname{Pr}\left(P U_{o n}\right)+1 \cdot \operatorname{Pr}\left(P U_{o f f}\right)
$$

where $\operatorname{Pr}\left(P U_{o n}\right)$ and $\operatorname{Pr}\left(P U_{o f f}\right)$ represent the probability of the PU being in on/off state, which can be learned statistically over long term via temporal spectrum sensing. To analyze $P_{O T}$, the only term that remains to be assessed is $P_{S C T}$, which is the focus of this paper. The probability of successful concurrent transmission can therefore be used as a measure of the gain achieved in spectrum utilization, by reusing PU bands.

Further we consider two scenarios: Downlink and Uplink for our analysis since both differ in their location estimation or sensing requirements, as discussed later. We shall assume that all transmissions are omni directional and the signal propagation is governed by a $\log$ normal shadowing model. The received power at node $i\left(P_{i}\right)$ due to node $j$ can be expressed as:

$$
P_{i}=s_{j}-g\left(d_{i, j}, n_{i, j}\right)+W[\mathrm{dBm}]
$$


where $s_{j}$ represents the transmission power of node $j$ and $d_{i, j}$ denotes the distance between nodes $i$ and $j$. Further

$$
p l g(d, n)=10 n \log _{10}\left(d / d_{o}\right)[\mathrm{dB}]
$$

and denote it by $g(d)$, where $d_{o}$ is the reference distance. We assume that shadowing noise $W \sim\left(0, \sigma_{w}^{2}\right)$ where $\sigma_{w}^{2}$ denotes the shadowing variance in $\mathrm{dB}$.

The goal of this work is to evaluate 4.2 for both $L A S$ and $L U S$ in order to shed light on advantages of embedding location awareness in CR nodes. Before we proceed, we present an analytical result that will be useful for our ensuing analysis.

Result: If $\mathrm{X}$ and $\mathrm{Y}$ are two normally distributed random such that $\mathrm{X} \sim\left(\mu_{x}, \sigma_{x}^{2}\right)$ and $\mathrm{Y} \sim\left(\mu_{y}, \sigma_{y}^{2}\right)$. Then, the probability that ratio of $\mathrm{X}$ and $\mathrm{Y}$ exceeds a constant $\mathrm{T}(T \gtrless 0)$ i.e., $\operatorname{Pr}(X / Y) \geq T$ is given by:

$$
\operatorname{Pr}(X / Y) \geq T=Q\left(\left(-\mu_{z}\right) / \sigma_{z}\right)
$$

where $\mu_{z}=\mu_{x}+T \mu_{y}$ and $\sigma_{z}=\sqrt{\left(\sigma_{x}^{2}+T^{2} \sigma_{y}^{2}\right)}$ and $\mathrm{Q}$ function can be defined as:

$$
Q(x)=1 / 2(1-\operatorname{erf}(x / \sqrt{2}))
$$

\subsection{Downlink Analysis}

In the downlink, a BS tries to establish connections with active MSs within its coverage area, using wireless channels. In the current setup we assume $M$ PUs to be active at any time, each of which is connected to the BS via an orthogonal frequency band. The BS is equipped with ATPC, which enables it to adjust its transmission power in accordance to its distance from the intended MS.

Concurrently CR nodes seek to form single hop links with their intended receivers, by reusing one of the M PU bands. However, a $\left(C R_{T x}, C R_{R x}\right)$ pair overlays with $\mathrm{PU}$ transmission on a common frequency band, if only when this CR link confirms that 4.1 is satisfied. If 4.1 is not satisfied for a particular band, the $\mathrm{CR}$ pair continues to scan the remaining $M-1 \mathrm{PU}$ bands until 4.1 is satisfied for any one of them. If 4.1 is not satisfied for neither of the $M$ PU bands, the CR pair fails to establish a link.

\subsubsection{Location Aware Scenario (LAS)}

Under the $L A S$ we assume that the CR nodes are aware of the locations of PU transmitter (BS) and PU receivers (MSs) along with their characteristic frequency bands. The 


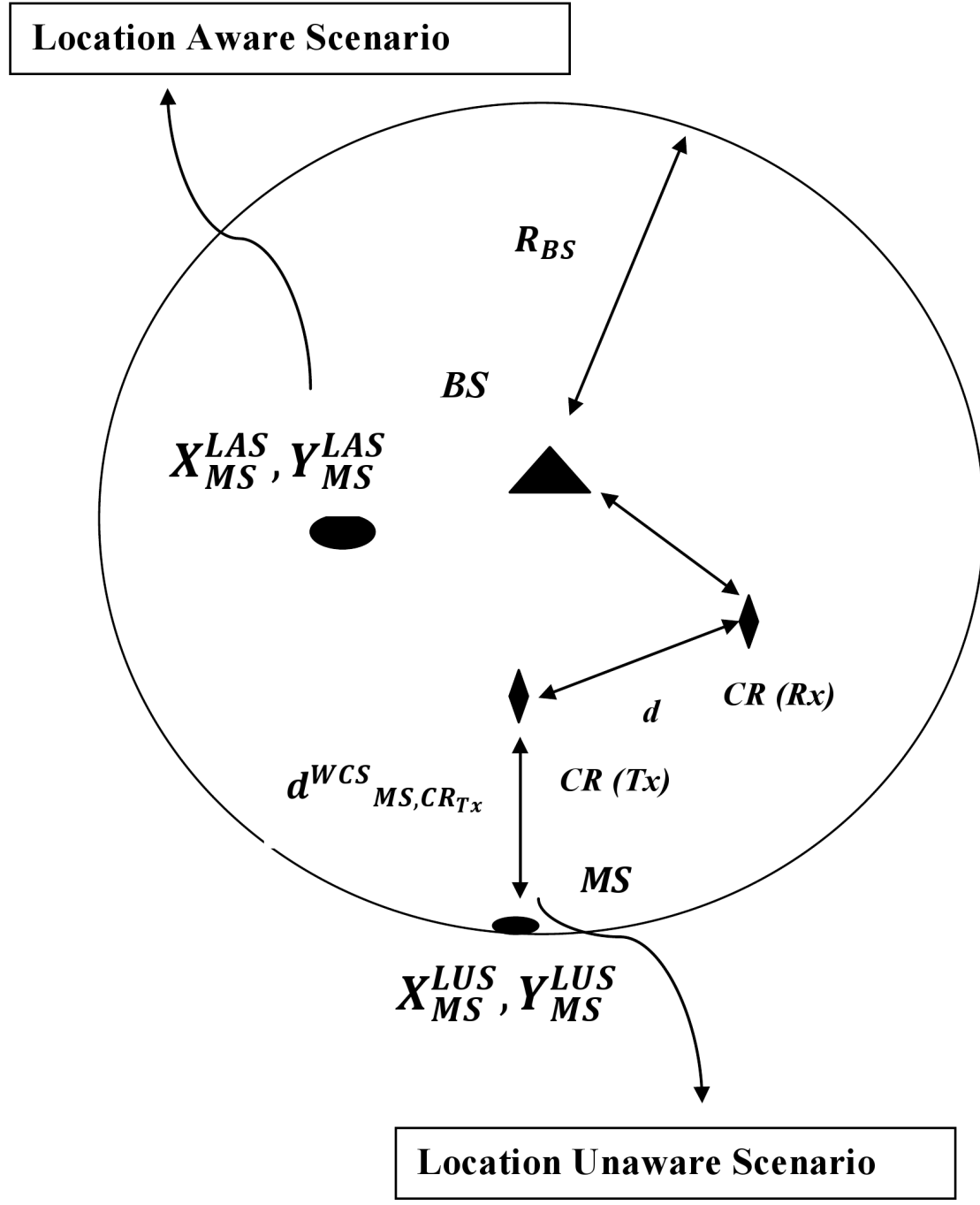

Figure 4.1 - System Model for location unaware (LUS) and location aware (LAS) scenarios 
assumption regarding prior information of the location of BS and available frequency bands is reasonable because BSs for PNs (such as cellular, TV) are fixed and have distinct frequency ranges. Information concerning location of MSs can either be acquired either by collaboration with the PN or cooperation among CR nodes as discussed in chapter 2. Information regarding occupancy status PU bands can be acquired by spectrum sensing.

Before a CR pair $\left(C R_{T x}, C R_{R x}\right)$ begins transmitting concurrently with a PU transmission, it sequentially validates 4.1 for the available $M$ PU bands. As soon as 4.1 becomes true for a band, that band is reused by the CR network.

Let $k$ represent the PU bands, such that $k=1,2, \ldots . M$. Here, we describe the computation for a single PU band, which can be extended to the remaining $M-1$ PU bands. Consider a particular case when a CR pair $\left(C R_{T x}, C R_{R x}\right)$ comprising of a $C R_{T x}$ located at a distance $r^{\prime}$ from the $\mathrm{BS}$ and its intended $\mathrm{CR}$ receiver $\left(C R_{R x}\right)$, choose to validate 4.1 for the $k^{\text {th }} \mathrm{PU}$ band, currently occupied by a $\mathrm{MS}_{k}$ present at $X_{M S_{k}}^{L A S}, Y_{M S_{k}}^{L A S}$ (Fig.1).

In order to validate 4.1, we need to estimate the signal and interference powers received from the transmitters (BS and $C R_{T x}$ ) at the receivers $\left(\mathrm{MS}_{k}\right.$ and $C R_{R x}$ ) for both the CR ad hoc and PN. Using (3-5) we get:

$$
P_{\min , M S}=P_{B S}-10 n \log _{10}\left(d_{M S_{k}, B S} / d_{o}\right)[\mathrm{dBm}]
$$

where $P_{B S}$ denotes the transmission power of the BS subject to a minimum mean received power $\left(P_{\min , M S}\right)$ at $\mathrm{MS}_{k}$. Power received at $C R_{R x}$ from $C R_{T x}$ is given by:

$$
P_{C R_{R x}}=P_{C R_{T x}}-10 n \log _{10}\left(d / d_{o}\right)+W[\mathrm{dBm}]
$$

where $P_{C R_{T x}}$ denotes the transmission power of the $C R_{T x}, d$ is the distance between the $C R_{T x}$ and $C R_{R x}$ pair. Interference powers at $C R_{R x}\left(P_{C R_{R x}}^{I}\right)$ and $\mathrm{MS}_{k}\left(P_{M S_{k}}^{I}\right)$ are dependent on $r^{\prime}$ because as $r^{\prime}$ increases, the $C R_{T x-R x}$ moves farther from the $\mathrm{PN}$ and is bound to cause and receive less interference:

$$
\begin{gathered}
P_{C R_{R x}}^{I}\left(r^{\prime}\right)=P_{B S}-10 \log _{10}\left(d_{B S, C R_{R x}} / d_{o}\right)+W[\mathrm{dBm}] \\
P_{M S_{k}}^{I}\left(r^{\prime}\right)=P_{C R_{T x}}-10 \log _{10}\left(d_{M S_{k}, C R_{T x}} / d_{o}\right)+W[\mathrm{dBm}]
\end{gathered}
$$

Signal to Interference ratios for the CR pair $\left(S_{A} R_{A, L A S}^{D}\right)$ and PU $\left(S I R_{I, L A S}^{D}\right)$ can be expressed as:

$$
\begin{aligned}
& S_{I} R_{I, L A S}^{D}\left(r^{\prime}\right)=P_{\min , M S} / P_{M S_{k}}^{I}\left(r^{\prime}\right) \\
& \operatorname{SIR}_{A, B C S}^{D}\left(r^{\prime}\right)=P_{C R_{R x}} / P_{C R_{R x}}^{I}\left(r^{\prime}\right)
\end{aligned}
$$

For downlink, 4.2 can be expressed as:

$$
\begin{aligned}
& P_{S C T, L A S}^{D}\left(r^{\prime}\right) \\
& =P\left(\left(\operatorname{SIR}_{I, L A S}^{D}\left(r^{\prime}\right)>\operatorname{SIR}_{I, T}^{D}\right) \cap\left(\operatorname{SIR}_{A, L A S}^{D}\left(r^{\prime}\right)>\operatorname{SIR}_{A, T}^{D}\right)\right)
\end{aligned}
$$


where $\operatorname{SIR}_{I, T}^{D}$ and $\operatorname{SIR}_{A, T}^{D}$ denote the downlink SIR thresholds for primary and ad hoc networks respectively. Since the CR ad hoc and primary network operate independent of each other, $P_{S C T, L A S}^{D}$ can be considered as a product of two independent probabilities:

$$
\begin{aligned}
& P_{S C T, L A S}^{D}\left(r^{\prime}\right) \\
= & P\left(\operatorname{SIR}_{I, L A S}^{D}\left(r^{\prime}\right)>\operatorname{SIR}_{I, T}^{D}\right) \cdot P\left(\operatorname{SIR}_{A, L A S}^{D}\left(r^{\prime}\right)>\operatorname{SIR}_{A, T}^{D}\right)
\end{aligned}
$$

Evidently, the expression in 4.13 is a function of system parameters $\left(\operatorname{SIR}_{I, T}^{D}, \operatorname{SIR}_{A, T}^{D}\right.$, $\left.P_{\min , M S}, P_{C R_{T x}}, P_{B S}, M, R\right)$ and channel parameters $\left(n, \sigma_{w}^{2}\right)$. Therefore it is logical to express it as a conditional probability. We use 4.4 to arrive at the analytical expression for conditional probability:

$$
\begin{aligned}
& P_{S C T, L A S}^{D}\left(r^{\prime}\right)= \\
& Q\left(\frac{-\left(P_{M i n, M S}-T .\left(P_{M S}^{I}\left(r^{\prime}\right)\right)\right)}{\sqrt{\sigma_{w}^{2}+T^{2} \sigma_{w}^{2}}}\right) \\
& \times Q\left(\frac{-\left(P_{C R_{R x}}-T^{\prime} \cdot\left(P_{C R_{R x}}^{I}\left(r^{\prime}\right)\right)\right)}{\sqrt{\sigma_{w}^{2}+T^{2} \sigma_{w}^{2}}}\right)
\end{aligned}
$$

where $T$ and $T^{\prime}$ represent downlink SIR thresholds for the ad hoc and PN respectively. Even though 4.14 involves evaluating $\mathrm{Q}$ functions, precise numerical values can be obtained by plugging in values of system and channel parameters and, CR and MS locations. For the current setup, CR nodes and $M S s$ are uniformly distributed within a radius $R$, with the $B S$ at the center. The average performance can be obtained by.

$$
P_{a v g, S C T(L A S)}^{D}=\int_{0}^{R} P_{S C T, L A S}^{D}\left(r^{\prime}\right) \cdot r^{\prime} \cdot d r^{\prime}
$$

Due to dependence of 4.15 on system design and channel parameters, it can be used to evaluate impact of critical design parameters on system performance of $\mathrm{CR}$ overlaid $C R$ networks. Given the closed form expression in 4.14 , the integral in $4.15 \mathrm{can}$ be evaluated numerically.

\subsubsection{Location Unaware Scenario (LUS)}

Under the $L U S$, the CR nodes are aware of only the location of the PU transmitter (a.k.a BS). Similar to the LAS 4.1 needs to be validated for the $k^{\text {th }}$ PU band. This is done by assuming the PU receiver $\left(M S_{k}\right)$ to be located at $\left(X_{M s_{k}}^{L U S}, Y_{M s_{k}}^{L U S}\right)$ as shown in Fig 4.1 . Evidently the PU receiver (MS) is assumed to be present at the fringe of the coverage area, closest to the $C R_{T x}$. This is a far more conservative approach as compared to $L A S$. 
Similar to LAS, SIR for the CR ad hoc $\left(\operatorname{SIR}_{A, L U S}^{D}\right)$ and PN $\left(\operatorname{SIR}_{I, L U S}^{D}\right)$ are dependent of $r^{\prime}$, and can be expressed as:

$$
\begin{gathered}
\operatorname{SIR}_{A, L U S}^{D}\left(r^{\prime}\right)=P_{C R_{R x}} / P_{C R_{R x}}^{I}\left(r^{\prime}\right) \\
\operatorname{SIR}_{I, L U S}^{D}\left(r^{\prime}\right)=P_{M i n, M S} / P_{M S_{k}}^{I}\left(r^{\prime}\right)
\end{gathered}
$$

For downlink under the LUS 4.2 can be expressed as:

$$
\begin{aligned}
& P_{S C T, L U S}^{D} \\
= & P\left(\operatorname{SIR}_{I, L U S}^{D}\left(r^{\prime}\right)>S I R_{I, T}^{D}\right) \cdot P\left(\operatorname{SIR}_{A, L U S}^{D}\left(r^{\prime}\right)>S I R_{A, T}^{D}\right)
\end{aligned}
$$

We use 4.4 to arrive at the analytical expression for conditional probability:

$$
\begin{aligned}
& P_{S C T, L U S}^{D}\left(r^{\prime}\right)= \\
& Q\left(\frac{-\left(P_{\min , M S}-T .\left(P_{M S}^{I}\left(r^{\prime}\right)\right)\right)}{\sqrt{\sigma_{w}^{2}+T^{2} \sigma_{w}^{2}}}\right) \\
& \times Q\left(\frac{-\left(P_{C R_{R x}}-T^{\prime} \cdot\left(P_{C R_{R x}}^{I}\left(r^{\prime}\right)\right)\right)}{\sqrt{\sigma_{w}^{2}+T^{2} \sigma_{w}^{2}}}\right)
\end{aligned}
$$

Meanwhile 4.15 for $L U S$ becomes:

$$
\begin{aligned}
& P_{\text {avg,SCT,LUS }}^{D}= \\
= & \int_{0}^{R} P_{S C T, L U S}^{D}\left(r^{\prime}\right) r^{\prime} d r^{\prime}
\end{aligned}
$$

Like 4.15, we can use 4.20 to evaluate impact of system design and channel parameters on average system performance of location unaware CR overlaid networks for downlink. Numerical methods can be used to attain precise values for 4.20 .

\subsection{Uplink Analysis}

In uplink, MSs try to establish a connection with the catering BS using their characteristic wireless channels. Simultaneously CR nodes present within the same area seek to establish peer to peer links with their neighbors by reusing primary frequency bands. Here, MSs are equipped with (ATPC), which enables them to adjust their transmission power in accordance to their distance from the $B S$. Let $P_{\min , B S}$ denote the required minimum received power at the BS. Then $M S_{k}$ transmits at $P_{M S_{k}}$ such that:

$$
P_{M S_{k}}=P_{\min , B S}+L_{M S_{k}, B S}
$$


The critical difference between the $L A S$ and $L U S$ for downlink was defined by the absence of location information of PU receivers (MSs), by the CR network in LUS. However, in the uplink localization of MSs is not a challenge, as they can be localized using methods outlined in chapter 2. In addition, location of the BS, which in this case is the receiver is known apriori. Therefore, unlike the downlink scenario, we only have the LAS scenario for uplink.

\subsubsection{Location Aware Scenario}

Following a procedure similar to downlink, we illustrate the computation for the $k^{\text {th }} \mathrm{PU}$ band, by a $\left(C R_{T x}, C R_{R x}\right)$ pair. Expressions for $\operatorname{SIR}_{I, T}^{U}\left(r^{\prime}\right)$ and $\operatorname{SIR}_{A, T}^{U}\left(r^{\prime}\right)$ are expressed as:

$$
\begin{aligned}
& \operatorname{SIR}_{I, L A S}^{U}\left(r^{\prime}\right)=P_{\min , B S} / P_{B S}^{I}\left(r^{\prime}\right) \\
& \operatorname{SIR}_{A, L A S}^{U}\left(r^{\prime}\right)=P_{C R_{R x}} / P_{C R_{R x}\left(r^{\prime}\right)}^{I}
\end{aligned}
$$

where $P_{B S}^{I}$ represents the interference power received at the BS from $C R_{T x}$ on the $k_{t h} \mathrm{PU}$ band, and is given by:

$$
P_{B S}^{I}\left(r^{\prime}\right)=s C R-10 n \log _{10}\left(r^{\prime} / d_{o}\right)+W[\mathrm{dBm}]
$$

Here, $P_{C R_{R x}}^{I}$ represents the received interference power received by the $C R_{R x}$ from the MS on the $k^{\text {th }} \mathrm{PU}$ band, and can be expressed as:

$$
P_{C R_{R x}}^{I}\left(r^{\prime}\right)=s B S-10 n \log _{10}\left(d_{M S, C R_{R x}} / d_{o}\right)+W[\mathrm{dBm}]
$$

For uplink 4.2 can be expressed as:

$$
\begin{aligned}
& P_{S C T, L A S}^{U} \\
= & P\left(\operatorname{SIR}_{I, L A S}^{U}\left(r^{\prime}\right)>\operatorname{SIR}_{I, T}^{U}\right) \cdot P\left(\operatorname{SIR}_{A, L A S}^{U}\left(r^{\prime}\right)>\operatorname{SIR}_{A, T}^{U}\right)
\end{aligned}
$$

where $\operatorname{SIR}_{I, T}^{U}$ and $\operatorname{SIR}_{A, T}^{U}$ represent the respective uplink thresholds for the PN and the CR ad hoc network respectively.

Using 4.4 we derive at the analytical expression for the conditional probability $\left(P_{S C T, L A S}^{U}\left(r^{\prime}\right)\right)$

$$
\begin{aligned}
& Q\left(\frac{-\left(P_{M i n, M S}-T .\left(P_{M S}^{I}\left(r^{\prime}\right)\right)\right)}{\sqrt{\sigma_{w}^{2}+T^{2} \sigma_{w}^{2}}}\right) . \\
\times & Q\left(\frac{-\left(P_{C R_{R x}}-T^{\prime} .\left(P_{C R_{R x}}^{I}\left(r^{\prime}\right)\right)\right.}{\sqrt{\sigma_{w}^{2}+T^{2} \sigma_{w}^{2}}}\right)
\end{aligned}
$$


Meanwhile 4.15 for $L A S$ becomes:

$$
P_{a v g, L A S}^{U}=\int_{0}^{R}\left(P_{c o n d, S C T, L A S}^{U}\left(r^{\prime}\right)\right) r^{\prime} d r^{\prime}
$$

4.26 can prove to be a useful performance metric to evaluate impact of system design and channel parameters on system performance of CR overlaid networks for uplink and can be evaluated using numerical methods.

\subsection{Numerical Results and Discussion}

In this section we present analytical and simulation results for $P_{a v g, S C T}$, which is used as a performance metric, for both uplink and downlink scenarios. Impact of wireless channel parameters (Path loss and Shadowing) and key system design parameters (detection thresholds, system capacity, coverage area and ATPC) is examined on $P_{a v g, S C T}$. In the end we draw conclusions based on our findings.

\subsubsection{Simulation setup}

Our test setup is similar to Figure 4.1. The location of the BS is fixed at $X_{B S}, Y_{B S}$. Locations of $M \mathrm{MSs}$ are uniformly generated within a radius of $800 \mathrm{~m}\left(R_{B S}\right)$. Location of $C R_{T x}$ is represented as $\left(r^{\prime}, \theta_{a}\right)$, where $\left(r^{\prime}\right)$ is its distance from the primary BS and $\theta_{a}$ is uniformly distributed in $(0,2 \pi)$. Other key simulation parameters include path loss factor $(n)$, which is assumed to be 4 , reference distance $\left(d_{o}\right)$ is taken to be 100 $\mathrm{m}$, shadowing variance $\left(\sigma_{w}^{2}\right)$ is $4 \mathrm{~dB}$ and transmission power of $\mathrm{CR}$ nodes $\left(P_{C R_{T x}}\right)$ is 40 $\mathrm{dBm}$. Location of $C R_{R x}$ is generated uniformly within a radius $\left(\right.$ Range $\left._{C R}=100 \mathrm{~m}\right)$, with $C R_{T x}$ as the center. For a particular value of $r^{\prime}, 10000$ simulation trials were carried out.

Each simulation trial gave a new set of locations for $M S s, C R_{T x}$ and $C R_{R x}$, the above values were then used to compute an analytical $P_{S C T}$ using either 4.15 4.20 or 4.25 depending on the scenario. To arrive at the simulation value of $P_{S C T}$, the simulation was run 10,000 times for a given set, and $P_{S C T}$ was calculated by counting the number of instances where 4.1 was true. In the end, analytical and simulated values from all the 10,000 sets, for a given $r^{\prime}$ were averaged to get the analytical and simulated $P_{a v g, S C T}\left(r^{\prime}\right)$. Value of $r^{\prime}$ was varied from $100 \mathrm{~m}$ to $2000 \mathrm{~m}$ for $L A S$, and upto $3000 \mathrm{~m}$ for $L U S$. As can be seen from our Figs, the simulated and analytical results match to a great degree.

\subsubsection{Effect of System Capacity and Detection Threshold}

Fig. 4.2 and Fig. 4.3 depict the impact of the system capacity and the detection thresholds on $P_{a v g, S C T}$ for uplink and downlink (LAS, LUS) respectively. System capacity 


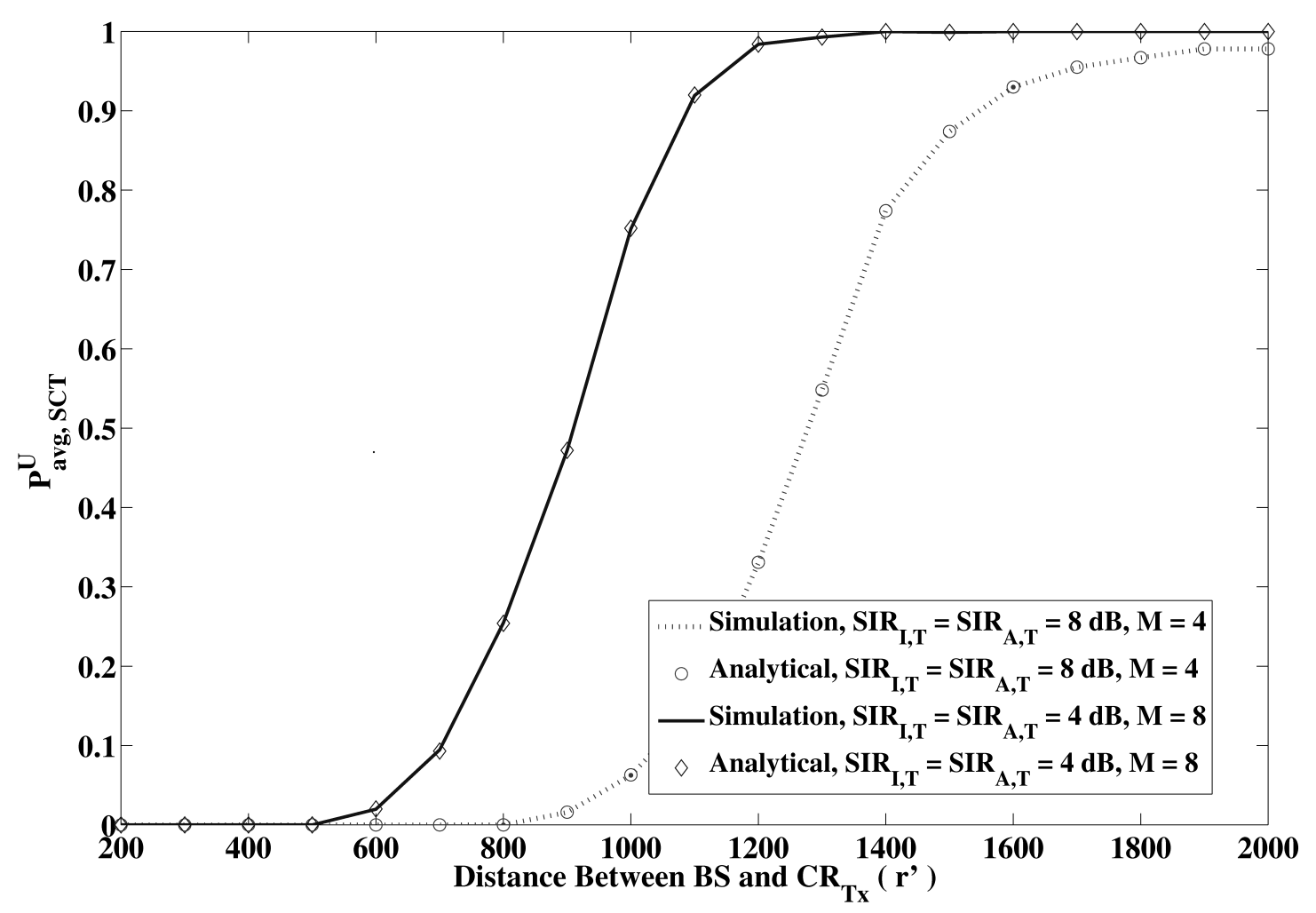

Figure 4.2 - Impact of increasing system capacity (M) and decreasing detection thresholds on $P_{a v g, S C T}^{U}$ against distance between $C R_{T x}$ and $\mathrm{BS}\left(r^{\prime}\right)$

here is defined as the number of active PU bands $M$, available for reuse by the $\mathrm{CR}$ ad hoc network. Evidently, $P_{\text {avg }, S C T}$ improves as $r^{\prime}$ increases for both uplink and downlink. As $r^{\prime}$ increases, the $\left(C R_{T x}, C R_{R x}\right)$ pair moves farther from the primary BS and MSs, thus causing and receiving less interference to and from the PN.

Meanwhile, as the number of reusable PU bands increase, $P_{\text {avg,SCT }}$ increases correspondingly. However, it can be observed that there is significant difference in the rate of increase of $P_{\text {avg }, S C T}$ between scenario $1\left(M=4, S I R_{I, T}=S I R_{A, T}=8 d B\right)$ and scenario $2\left(M=8, S I R_{I, T}=S I R_{A, T}=4 d B\right)$. Clearly, increasing $M$ and, decreasing $S I R_{I, T}$ and $S I R_{A, T}$ increases the system performance of the considered LAS overlaid CR network significantly. However, for the $L U S$ the $P_{a v g, S C T}$ is always almost zero because of a far more conservative approach. 


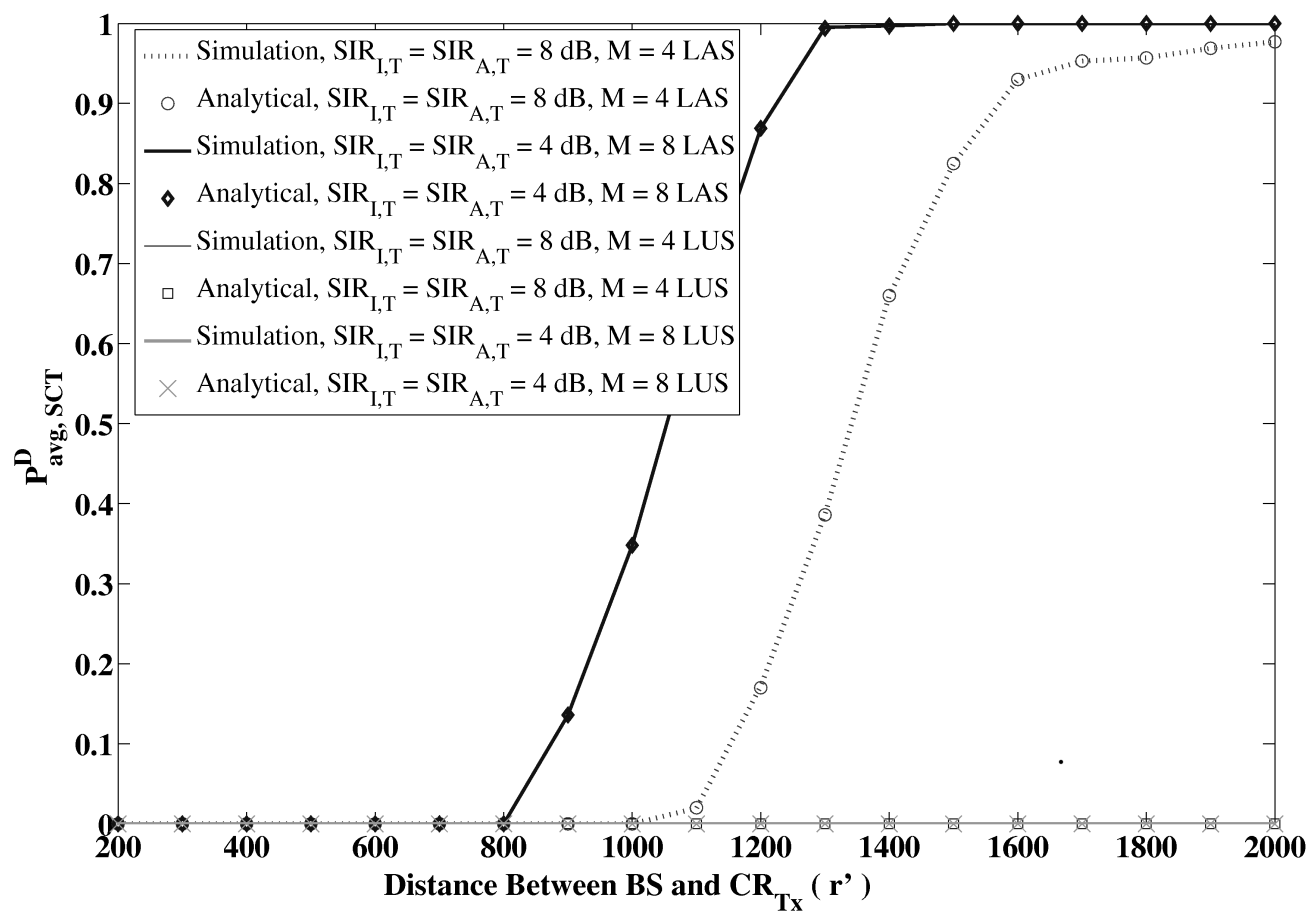

Figure 4.3 - Impact of increasing system capacity (M) and decreasing detection thresholds on $P_{a v g, S C T}^{D}$ against distance between $C R_{T x}$ and $\mathrm{BS}\left(r^{\prime}\right)$

\subsubsection{ATPC Parameters}

Fig. 4.4 and Fig. 4.5 depict the impact of minimum power requirements for the PN $\left(P_{\text {min, Ms }}, P_{\min , \mathrm{Bs}}\right)$ on rate of growth of $P_{\mathrm{avg}, \mathrm{SCT}}$ for downlink and uplink respectively. Each of the figures presents a comparison between two scenarios. For downlink, $P_{\min , \mathrm{Ms}}$ is $30 \mathrm{dBm}$ for scenario 1 and $-10 \mathrm{dBm}$ for scenario 2 . Similarly, for uplink, $P_{\min , \mathrm{Bs}}$ is -30 $\mathrm{dBm}$ for scenario 1 and $-10 \mathrm{dBm}$ for scenario 2 .

With decrease in minimum power requirements the rate of improvement of $P_{\text {avg, SCT }}$ increases significantly. This difference in system performance can be explained as follows: upon reducing the minimum power level requirements for the PU bands, the interference power level at the $C R_{R x}$ decreases, thereby increasing the chances of coexistence of PU and CR users on overlapping frequency bands.

\subsection{Conclusion}

In this work, we present analytical results for a general setup involving a CR ad hoc network, overlaid on a multi-channel and multi-user primary network. Our analytical and 


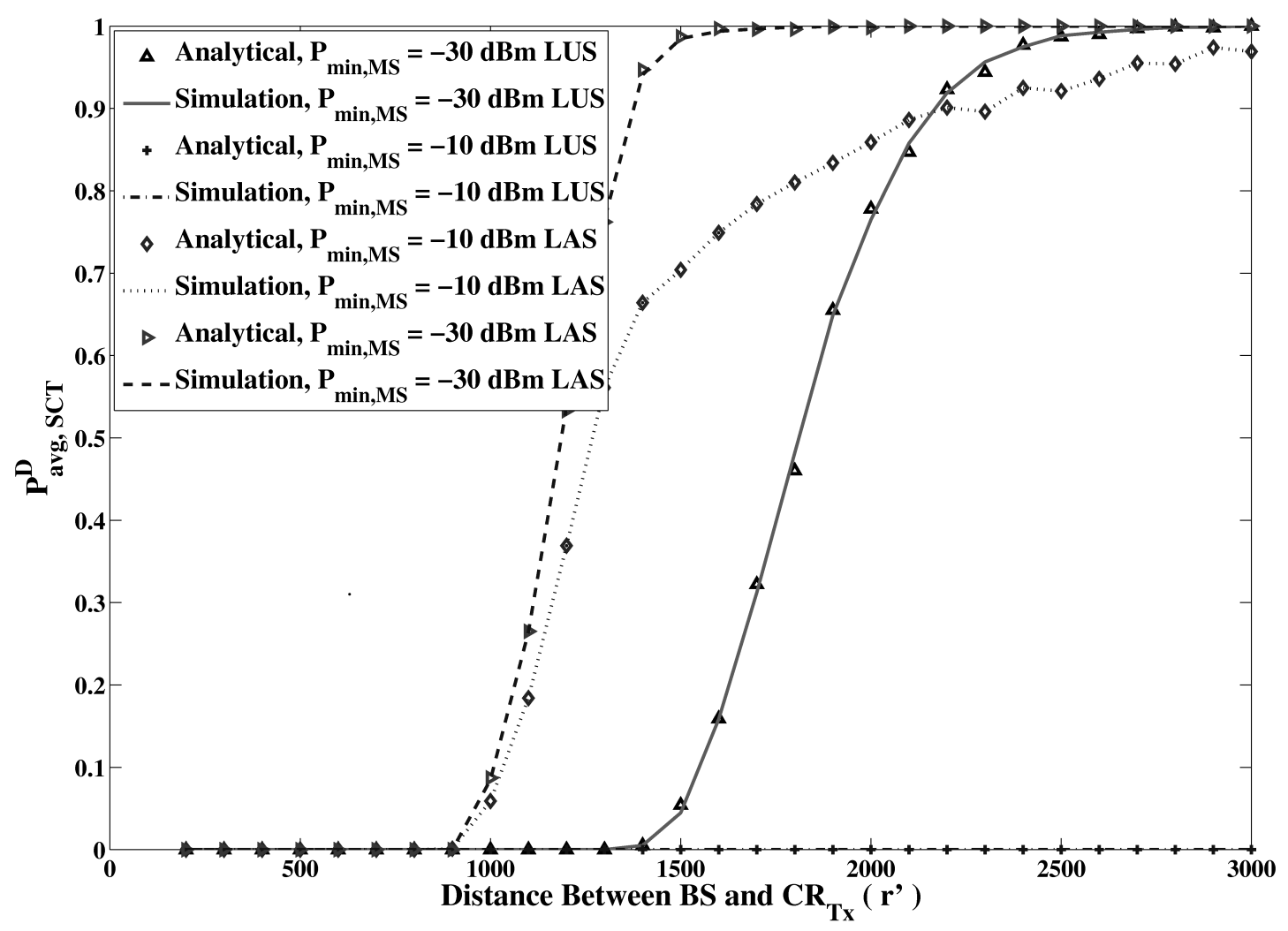

Figure 4.4 - Impact of decreasing minimum power requirement $\left(P_{\min , M S}\right)$ on $P_{a v g, S C T}^{D}$ against distance between $C R_{T x}$ and $\mathrm{BS}\left(r^{\prime}\right)$

simulation results show that embedding location awareness in CR networks increases system performance significantly. Our work also sheds light on the impact of system design parameters on performance gain of overlaid CR ad hoc networks. It was found that on decreasing crucial system parameters such as minimum power requirements and detection thresholds and, increasing system capacity the chances of opportunistically reusing active PU bands increase. These key findings justify inclusion of location awareness in development of future wireless standards. Also, our findings motivate the development of signal processing algorithms that can enable communication systems to operate reliably in the low signal to interference environments. 


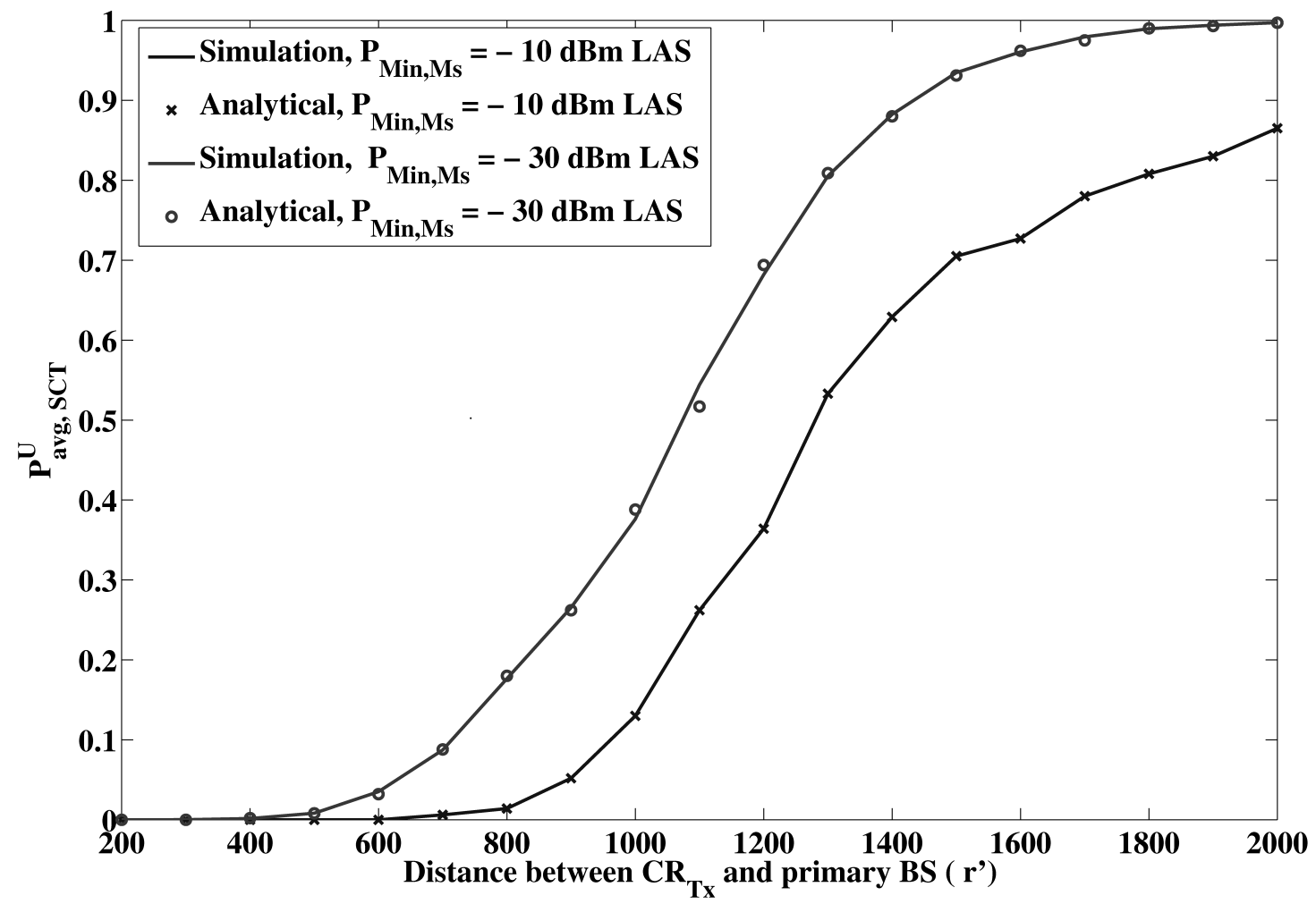

Figure 4.5 - Impact of decreasing minimum power requirement $\left(P_{\min , B S}\right)$ on $P_{a v g, S C T}^{U}$ against distance between $C R_{T x}$ and $\mathrm{BS}\left(r^{\prime}\right)$ 


\section{Chapter 5}

\section{Resource Management in CRNs - Multi CR User}

In this chapter motivated by our observations from the previous chapter, we extend our analysis to a more pragmatic scenario involving multiple CR and PU links. Here, as considered before a CR network is overlaid on an existing primary network (PN). The PN consists of a primary Base Station (PBS) which communicates with PUs, present within its coverage area in downlink mode, and is equipped with adaptive transmit power control (ATPC). The ATPC capability enables the BS to control its transmission power, in accordance to the distance from the intended receiver. This helps conserve power at the BS. Each of the primary receivers (PRs) is assigned a characteristic frequency channel for reception. We assume that the channel allocation strategy for the primary users remains unchanged during our process of resource management for CRNs. In order to contrast and compare the impact of location awareness on CR network performance we consider three different cases, varying in levels of location awareness: Full location Aware (FLA), Partial Location Aware (PLA) and No location Awareness (NLA).

1. Full location awareness: In FLA, we assume to have prior information concerning location of Primary Transmitters (PTXs), Primary Receivers (PRXs) and their corresponding channel occupancy. As will be seen later based on this prior information, we can accurately quantify interference caused by CR transmissions to primary receivers and achieve significantly higher system performance as compared to other scenarios, at the cost of higher system complexity.

2. Partial Location awareness: In PLA, we assume to have prior information concerning location of PTXs, primary receiver sensitivity thresholds and corresponding channel occupancy. Using our prior knowledge of primary receiver sensitivity, we estimate location of all primary receivers as described in Chapter 2. Thereafter, we quantify interference caused by $\mathrm{CR}$ transmissions to primary receivers based 
the estimated inter distances. It is clearly evident that degree of location awareness in PLA is significantly less as compared to FLA, thus making it more conservative toward exploiting spatial spectrum opportunities.

3. No Location Awareness: In NLA, we have no prior information concerning neither receiver sensitivity nor geographical location of primary receivers. The only available information is knowledge of location of PTX, which can be obtained using methods outlined in Chapter 2. Given the limited information and the stringent requirement to protect PUs, we adopt a conservative approach and do not reuse a PU channel, if its occupancy has been determined via spectrum sensing.

Next we carry out an in depth analysis of impact of network parameters such as no. of PU channels, no of CR nodes, distance between CR pairs, duty cycle of $\mathrm{PN}$ and nature of propagation environment (n-Path loss exponent) on the CRN performance for all three types of overlaid CRNs (FLA/PLA/NLA). Performance metrics for our analysis include: Average network throughput (mbps) and Number of admitted CR users. Our work focuses on two types of network management problems:

(a) Sum rate maximization

(b) Maximization of number of admitted users

\subsection{System Model and Problem Statement}

We consider a single cell scenario as depicted in Fig. 3.1. The cell consists of a set of PUs and CR pairs. The set of PUs includes Primary Receivers (PRs) and a Primary Base Station (PBS). The spectrum of interest is divided into $M$ orthogonal frequency channels through a multiple access scheme, such as FDMA. Each of the M channels is assigned to M PUs, such that exactly one channel is assigned to each PR. The primary BS communicates with PRs in the area using these channels. The PRs are considered to be always in receiving mode. As a note, our formulation and analysis is not applicable to cases where PUs employ multiple access schemes such as orthogonal frequency division multiple access (OFDMA) or code division multiple access (CDMA). Also, we consider only downlink scenario for our analysis since acquiring location awareness in uplink in fairly straightforward, as described in chapter 4.

Our system setup resembles a broadcast scenario, where the BS is continuously transmitting on different channels via FDMA and all PRs are actively receiving. Television broadcasting is one possible implementation of this setup.

Within the same area we have K CR pairs, each consisting of a CR transmitter $C R_{T x}$ and CR receiver $C R_{R x}$. These CR pairs as in single user case (chapter -3), seek to reuse 
primary frequency bands to establish reliable links, subject to interference constraints at the PRs. We consider a centralized setup, where CR pairs send their requests for bandwidth to a central authority (CA), which can either be a base station for the CR network or a central spectrum broker. The CA is responsible for formulating the resource management strategy, and has access to varying degrees of global information, in accordance to the nature of the CRN (FLA/PLA/NLA) as will be described in detail in the later part of this chapter. Further, we assume that all CR nodes are equipped with exactly one RF front end, and thus all admitted CR users are assigned exactly one situation. The assumption concerning a single RF front end is reasonable for situations, where hardware complexity is an issue.

Each of these CR pairs can either be idle or active, depending of whether it is admitted or not. For efficient operation the CRN resource management strategy should strictly follow the following two conditions:

1. R1: The total amount of interference caused by all CR transmissions to each PU should not exceed a predefined SIR threshold at all times.

2. R2: All admitted CR pairs should be guaranteed a minimum transmission rate i.e., received signal to interference ratio should be above a predefined threshold.

As will be shortly seen, the resource management problem can be formulated as a mathematical optimization problem consisting of an objective function and a number of constraints.

To facilitate the formulation of our optimization problems, we define a few preliminaries:

1. Signal Model: We adopt a AWGN signal model for our analysis:

$$
P_{j}=P_{i}-10 n \log _{10}\left(d_{i, j}\right)+N_{o}
$$

Consider a pair of nodes $i$ and $j$ separated by a distance $d_{i, j}$. Here, $P_{j}$ represents the received power by node $j$ from node $i$, whose transmission power is $P_{i}$, and $N_{o}$ represents Gaussian noise, such that $N_{o} \sim N(0, \sigma)$, where $\sigma$ represents noise variance in $\mathrm{dB}$.

2. Adaptive Power Control for PUs: We assume that the PU network employs an adaptive power control strategy to control its transmission power, in accordance to its distance from the intended receiver. Let $P_{\min }$ denote the required minimum power at the primary receiver $P R$. So, for the $i^{t h} P R$ operating on the $i^{t h}$ PU band $\left(P R_{i}\right)$, the required transmission power can be expressed as:

$$
P B S_{i}=P_{\min }+10 n \log _{10}\left(d_{i}\right)-N_{o} \quad \forall i \varepsilon M
$$


Where $d_{i}$ denotes the distance of $P R_{i}$ from the primary base station and $P B S_{i}$ denotes the transmission power of PBS on channel $i$.

3. Channel Assignment: We introduce the binary variable $a_{c}^{i}$ such that:

$$
a_{i}^{c}=\left\{\begin{array}{lc}
1 & \text { if channel } c \text { is assigned to CR pair } i \\
0 & \text { if channel } c \text { is not assigned to CR pair } i
\end{array}\right.
$$

Further, we define two constraints based on $a_{c}^{i}$.

(a) C1: Since each CR pair $i$ is assigned a maximum of one PU channel, we have:

$$
\sum_{c=1}^{M} a_{i}^{c} \leq 1 \quad \forall \quad i \varepsilon K
$$

(b) C-2: Also we only assign a PU band $m$ to a maximum of one CR pair, we have:

$$
\sum_{i=1}^{K} a_{i}^{c} \leq 1 \quad \forall \quad c \varepsilon M
$$

4. Power Control: We denote the power level for CR pair $i$, corresponding to channel $c$ as $P_{i}^{c}$ such that:

$$
P_{i}^{c}=\left\{\begin{array}{l}
\varepsilon\left[0, P_{\max }\right] \quad \text { if } \quad a_{i}^{c}=1 \\
0 \quad \text { if } a_{i}^{c}=0
\end{array}\right.
$$

These conditions can be mathematically expressed as:

$$
P_{i}^{c} \leq a_{i}^{c} \cdot P_{\max }
$$

Since $P_{i}^{c}$ is always greater than zero, this condition ensures that if $a_{i}^{c}$ equals zero,then $P_{i}^{c}$ also equals zero.

\section{Protection to Primary Users:}

The main concern in assigning frequency channels and power levels to CR pairs is protection of PUs. Since PUs are licensed to operate within the spectrum of interest and always have the right of way, an efficient resource management strategy should aim to protect the PRs present in the area. The protection of PRs can be guaranteed by restricting CR power levels, so that the SIR thresholds of PRs $\left(S_{P R}\right)$ are not violated. 
This condition can be mathematically expressed as:

$$
P_{\min }-\left(\sum_{i=1}^{K} P_{j}^{m}-\sum_{i=1}^{K} G_{i, m}+N_{o}\right)-\sum_{i=1}^{K} \delta_{i}^{m} \geq T-\sum_{i=1}^{K} a_{i}^{m} \delta_{i}^{m}
$$

where

$$
\delta_{i}^{m}=-G_{i, m}
$$

Here $\delta_{i}^{m}$ ensures that interference to $P R_{m}$ is taken into account only from CR pair $i$ operating on band $m$. This can be explained in detail as follows:

If a CR pair is not admitted its transmission power should also be zero i.e., if $a_{c}^{i}=0$ then $P_{c}^{i}=0$. This is condition is ensured by 5.6. However, on examining 5.7 it can be seen that, if both $a_{c}^{i}$ and $P_{c}^{i}$ are equal to zero for a CR pair $i$ the term $G_{i, m}$ is still present. Therefore, it is essential to get rid of gains from CR pairs that have been denied entry.

Introducing the term $\delta$ on both sides of 5.7 helps avoid these terms and ensures that interference received at $P R_{m}$ is only taken into account from CR pairs operating on band $m$.

6. Minimum Rate guarantee to Admitted CR Users:

The received SIR at a CR receiver $i$ operating on band $c$ can be expressed as:

$$
S I R_{i}^{c}=\frac{P_{i}^{c}-G_{i i}}{\left(\left(\sum_{j=1, j \neq i}^{k} P_{j}^{c}-\sum_{j=1, j \neq i}^{N} G_{j, i}\right)+P B S_{c}-G_{B S, i}+N_{o}\right)} \quad \forall i \varepsilon K, c \varepsilon M
$$

Since all admitted CR are guaranteed a minimum rate, which is dictated by the $S I R_{C R}$ level at the CR receiver, therefore:

$$
\begin{aligned}
& S I R_{i}^{c} \geq T^{\prime} \\
& P_{i}^{c}-G_{i i}-\left(\left(\sum_{j=1, j \neq i}^{k} P_{j}^{c}-\sum_{j=1, j \neq i}^{N} G_{j, i}\right)+\right. \\
& \left.P B S_{c}-G_{B S, i}+N_{o}\right)-\sum_{j=1, j \neq i}^{K} \lambda_{j}^{c} \geq T^{\prime}-\sum_{j=1, j \neq i}^{K} a_{j}^{c} \lambda_{j}^{c}
\end{aligned}
$$

and

$$
\lambda_{j}^{c}=G_{j, i}
$$

Here $T^{\prime}$ is the minimum required SIR level at the CR receiver corresponding to each admitted CR pair. Here, the term $\lambda$ in 5.9 does the same function as $\delta$ in 5.7 . 
Next we apply tools from graph and optimization theory to solve our two resource management problems: Sum Rate Maximization and Maximization of Admitted CR users. Further, we carry out an in depth analysis of impact of system design parameters on the solutions of these problems, followed by drawing conclusions, which may form basis for design of future wireless standards.

\subsection{Sum Rate Maximization}

Sum rate maximization (SRM) aims at maximizing the aggregate rate of all admitted users, subject to resource and interference constraints. For our case SRM aims at maximizing aggregate rate of all admitted CR users, subject to available resources (Transmission power, frequency channel) and PR interference constraints.

The problem of resource management (RM) aims at assigning frequency channels and respective transmission powers to CR pairs, subject to interference and power constraints. Here, constraints are defined by minimum SIR thresholds, maximum allowed transmission power levels and number of frequency channels. The RM can be formulated as an optimization problem, with frequency and power as optimization variables.

Although works such as [33] have proposed allocation strategies for assigning multiple frequency bands to a CR pairs, we assume that any CR pair can be assigned a maximum of one PU band. This seems to be a more realistic situation as far as conservation of energy is concerned. Also, formulation of RM strategy under the condition of multi-channel assignment leads to a NP hard problem [34], whose complexity grows exponentially with the number of users and available channels. Further, we propose a centralized non iterative sum rate maximization algorithm based on Hungarian Algorithm, which has polynomial complexity.

The SRM can be mathematically expressed as:

$$
\operatorname{Maximize}_{a_{i}^{c} \varepsilon(0,1), P_{i}^{c}} \sum_{i=1}^{K} \sum_{c=1}^{M} \log _{2}\left(1+a_{i}^{c} \cdot S_{i}^{c}\right) \quad \forall i \varepsilon K, c \varepsilon M
$$

subject to:

$$
\begin{gathered}
\sum_{c=1}^{M} a_{i}^{c} \leq 1 \quad \forall \quad i \varepsilon K \\
\sum_{i=1}^{K} a_{i}^{c} \leq 1 \quad \forall \quad c \varepsilon M \\
P_{\text {min }}-\left(\sum_{i=1}^{K} P_{i}^{c}-\sum_{i=1}^{K} G_{i, c}+N_{o}\right)-\sum_{i=1}^{K} \delta_{i}^{c} \geq T-\sum_{i=1}^{K} a_{i}^{c} \delta_{i}^{c}
\end{gathered}
$$




$$
\begin{gathered}
P_{i}^{c}-G_{i i}-\left(\left(\sum_{j=1, j \neq i}^{k} P_{j}^{c}-\sum_{j=1, j \neq i}^{N} G_{j, i}\right)+P B S_{c}-G_{B S, i}+N_{o}\right)-\sum_{j=1, j \neq i}^{K} \lambda_{j}^{c} \geq T^{\prime}-\sum_{j=1, j \neq i}^{K} a_{j}^{c} \lambda_{j}^{c} \\
P_{i}^{c} \leq a_{i}^{c} . P_{\max }
\end{gathered}
$$

Since the assignment variables $a_{c}^{i}$ can either be 1 or 0 , and power variables $P_{c}^{i}$ can have any value in the interval $\left[0, P_{\operatorname{Max}}\right]$, this problem belongs to the class of MINLP (Mixed Integer Non Linear Programming) problems, which in general are NP hard. Optimal solution to these problems can be obtained using the branch and bound technique, which relies on solving a series of relaxed LP problems. However, complexity of these branch and bound based algorithms is exponential.

However, under the conditions 5.3 and 5.4, this MILP can be solved optimally using the Hungarian algorithm as discussed in the next section.

\subsection{Hungarian Algorithm}

Before we delve into the intricacies of the proposed algorithm for optimally solving 5.10 , we introduce a few terms related to graph theory which will be used in describing the algorithm.

"A graph $G=(V, E)$ consists of a set $V$ of vertices and a set $E$ of a pair of vertices called edges. For an edge $e=(u, v)$, we say that the endpoints of $e$ are $u$ and $v$ i.e., $e$ is incident to $u$ and $v$. A graph $G=(V, E)$ is bipartite if the vertex set $V$ can be partitioned into two sets $A$ and $B$, such that no edge in $E$ has both endpoints in the same set $(A, B)$ ".

"Weighted graphs are special kind of graphs in which each edge has an associated value known as weight, and are represented as $G(V, E, W)$ where $W$ represents the weight matrix. For a given edge its assigned weight can represent any characteristic such as cost, capacity or length etc".

Given a graph $G=(V, E)$, a matching $M$ in $G$ is a set of non adjacent edges i.e., no two edges share a common vertex. A given matching $M$ ' is known as maximal matching if it is not a proper subset of any other matching in graph $G$. That is, no edge can be added to $M^{\prime}$ without violating the matching condition.

"A matching $M$ is called maximum matching if it contains the largest possible number of edges. Also, note that every maximum matching is maximal, but the vice versa does not always hold true". 
For a weighted graph $G(V, E, W)$, a matching $M$ ” is known as maximum weighted matching if the sum of the weights of all included edges is maximum. A matching is called a perfect matching if all vertices are assigned a maximum of one edge. Note that all perfect matchings are maximum and thus maximal.

The maximum weighted bipartite perfect matching problem, also known as the assignment problem has received a great deal of attention due to its applicability in diverse fields. It can be stated as follows: given a set of workers, and set of ratings (weights) indicating how well each worker can perform each job, determine the best possible assignment of workers to jobs, such that total rating is maximized. Here, each worker is assigned a maximum of one job and each job is assigned to exactly one worker.

The rating of each worker corresponding to each job can be represented in the form of a rating matrix $R$, such that each element $r_{i, j}$ represents the rating(weight) of job $j$ being assigned to worker $i$.

The assignment problem can be expressed in linear program (LP) form as follows:

$$
\begin{array}{r}
L P: \\
\text { Maximize } \sum_{i, j} r_{i, j} \cdot x_{i, j} \\
\text { subject to : } \\
\sum_{j} x_{i, j}=1 \\
\sum_{i} x_{i, j}=1 \\
0 \leq x_{i, j} \leq 1
\end{array}
$$

Here $x_{i, j}$ represent binary variables, such that $x_{i, j}=1$ implies assignment of job $j$ to the $i^{t h}$ person and vice versa.

The optimal solution to the assignment problem is given by the Hungarian or KuhnMunkres algorithm, originally proposed by H.W Kuhn in 1955 and refined by J Munkres in 1975 [35]. The complexity of the Hungarian algorithm is upper bounded by $O\left(n^{3}\right)$ time, where $n$ is the size of one partition. Details concerning the Hungarian Algorithm and its functionality can be found in [35].

\subsubsection{Sum Rate Maximization based on Hungarian Algorithm}

It can noted that the graph connecting the set of CR pairs to the set of available frequency channels is a bipartite graph, thus our MILP optimization problem can be transformed to a maximum weighted perfect bipartite matching problem. 
Maximizing sum rate is equivalent to maximizing SIR for the CR pairs, which is same as maximizing transmission power of CR transmitters subject to PR interference constraints. Our Hungrian Algo. is similar in essence to SRM method mentioned in [36] . However, there exist several key difference concerning setup and available prior information. Further, the algorithm presented in [36] is an iterative algorithm unlike ours. Also, impact of location awareness has not been considered in [36]. Hungarian algo. is applicable to bipartite graphs i.e., a graph whose vertices can be divided into two disjoint sets $U$ and $V$ such that no edge in the same set are connected. In case of a weighted bipartite graph each of these edges has a weight $W$ associated with it.

Here, the sets $\mathrm{U}$ and $\mathrm{V}$ correspond to the CR pairs and available PU channels respectively. The edge weights are assigned in accordance to the criteria described in the following steps.

The Hungarian Algo. based SRM has the following key steps: Step 1: Maximum Power Calculation

As pointed out earlier maximization of throughput is equivalent to transmission power maximization, subject to PR interference constraints. We start my finding the maximum possible transmission power for every CR transmitter on every PU channel, given the PR SIR threshold.

$$
P_{i, j}^{U B}=P_{\min }-T+10 n \log _{10}\left(d_{i, j}\right)-N_{o}
$$

Where $d_{i, j}$ denotes the distance between the CR transmitter of $C R_{i}$ and $P R_{j}$. Step 2: Channel Set Selection

Given the $P_{i, j}^{U B}$ for all CR pairs and PU bands, the next step is to check if these transmission powers satisfy the power constraints and the individual rate demands. Every CR pair has a predefined maximum transmission power level, which is dependent on allowed power consumption, device characteristics etc. Therefore, the estimated upper bound on transmission power of each $\mathrm{CR}$ transmitter is compared with the maximum allowed transmit power level $\left(P_{\text {Max }}\right)$. Depending of whether it is greater or less than $\left(P_{\text {Max }}\right)$, we have the following two possibilities:

$$
\text { if } \begin{aligned}
&\left(P_{i, j}^{U B} \geq\right.\left.P_{\text {Max }}\right) \\
& P_{i, j}^{U B^{\prime}}= P_{\text {Max }} \\
& \text { else } \\
& P_{i, j}^{U B^{\prime}}=P_{i, j}^{U B}
\end{aligned}
$$

Here $P_{i, j}^{U B^{\prime}}$ denotes the modified upper bound on the transmit power of CR pair $i$ on PU channel $j$. 
Given $P_{i, j}^{U B^{\prime}}$ the SIR at the $\mathrm{CR}$ receiver corresponding to $\mathrm{CR}$ pair $i$, receiving on $\mathrm{PU}$ channel $j$ is calculated as:

$$
S I R_{i, j}=P_{i, j}^{U B^{\prime}}-10 n \log _{10}\left(d_{i}\right)-P B S_{j}+10 n \log _{10}\left(d_{B S, i}\right)-N_{o}
$$

Step 3: Weight Assignment

In order to apply HA to our setup we need a weight matrix $W$. The weights $w_{i, j}$ are assigned as follows:

$$
w_{i, j}=\left\{\begin{array}{l}
\log _{2}\left(1+\operatorname{SIR}_{i, j}\right) \quad \text { if } \quad \operatorname{SIR}_{i, j} \geq \operatorname{SIR} T \\
\infty \quad \text { if } \quad \operatorname{SIR} R_{i, j} \leq S I R_{T}
\end{array}\right.
$$

At the end of the third step we have a bipartite graph with weight matrix $W$, whose elements depict the edge weights.

If for a given CR and PU pair the edge is assigned a weight equal to infinity, it implies that the CR pair is prohibited from using that particular PU band for communication. However, it may be possible that the number of elements in the two bipartite sets i.e., set of CR users and set of available channels may not be equal, thus resulting in an unbalanced bipartite graph.

It is common practice to make the bipartite graph balanced before applying the Hungarian Algorithm [37], which is usually done by introducing dummy nodes [35]. Let the set of CR users be denoted by $U$ and the set of available primary channels by $V$. Our algorithm constructs two bipartite sets nodes each for the CR users and the available PU channels. The right set consists of $K$ nodes to represent the $K \mathrm{CR}$ users, and the left set consists of $M$ nodes to represent the $M$ PU channels.

If the number of $\mathrm{CR}$ users is greater than the number of PU channels, then the algorithm constructs $K$ - $M$ dummy nodes to be added in the left set as shown in Fig 5.1 However, if the number of PU channels is greater than the number of CR users, the algorithm appends $M-K$ dummy nodes to the right set 5.2. Each of the added nodes in a particular set is connected to all the nodes in the other set via edges, whose weights are assigned to be infinity. As a result of carrying out th above mentioned steps, we finally obtain a complete balanced weighted bipartite graph. Thus our proposed algorithm reduces the original SRM problem to a bipartite matching problem, which can be optimally solved using Hungarian Algorithm [38], whose codes are readily available for implementation. All edges whose weights are equal to infinity are treated as prohibited assignments and the Hungarian algorithm ensures that there exists no edge between the corresponding $\mathrm{CR}$ and PU pair in the final solution. 
Case 1: No. of PU Bands (m) > No. of CR pairs (k)

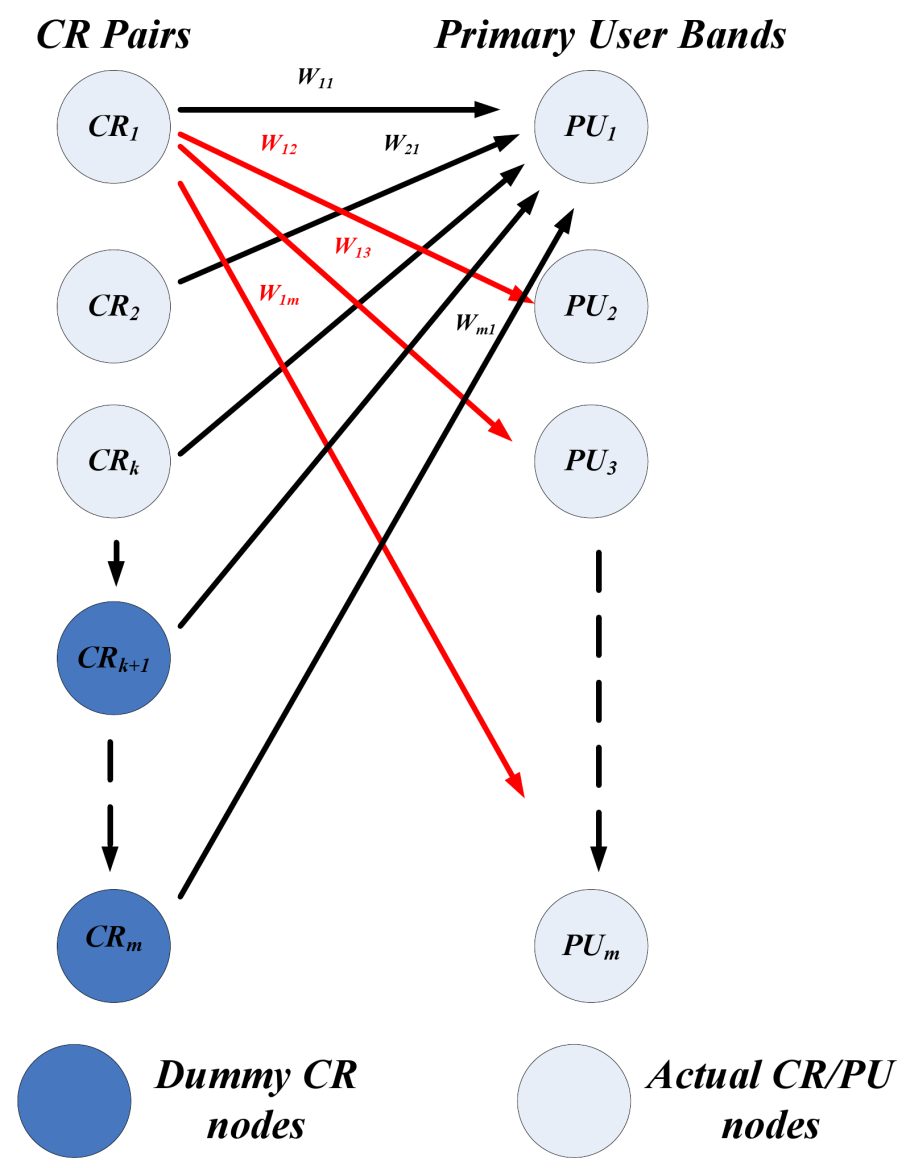

Figure 5.1 - Introduction of Dummy CR nodes for case where number of primary users is more than number of CR users 
Case 2: No. of PU Bands (m) < No. of CR pairs (k)

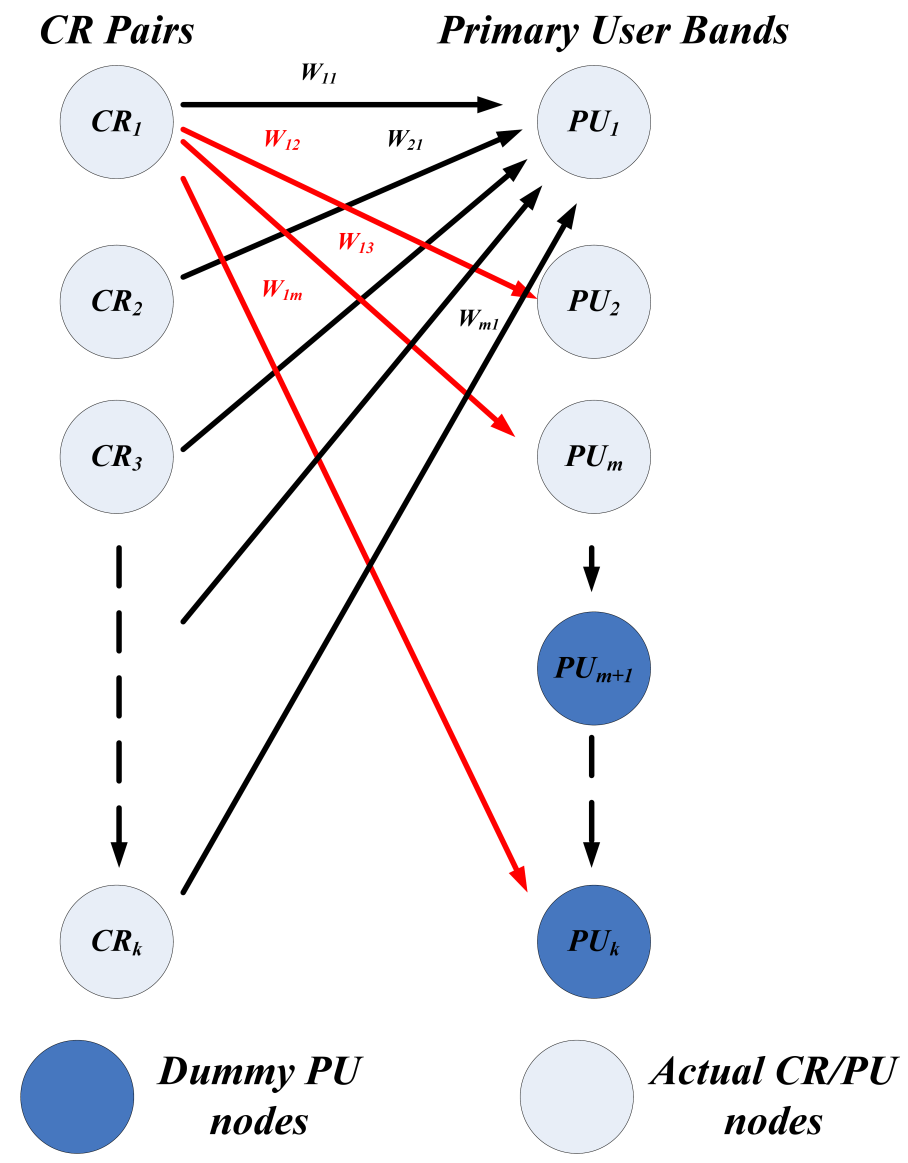

Figure 5.2 - Introduction of Dummy PU nodes for case where number of CR users is more than number of primary users 


\subsection{Maximization of Admitted Users (MAU)}

Delay sensitive applications such as real time video streaming and online gaming require high data rates i.e., admitted users should be guaranteed maximum possible rate subject to delay and interference constraints. This situation is similar to our setup in SRM.

However, there are situations where admission control aims at admitting the maximum number of users at minimum rate and at minimum transmission power. The rate in this case can be dictated by acceptable BER performance. Consider an example where we have CR pairs, which wish to transmit data which is delay insensitive such as short message service (SMS), thus requiring less data rate. In this case, our aim would be to admit maximum number of users at the specified minimum rate.

Here, all our constraints remain the same as compared to SRM, however our objective changes to reflect our intention of maximizing number of admitted users without considering maximization of assigned rate, as long as it is above our minimum threshold.

The optimization problem can be formulated as:

$$
\operatorname{Maximize} \sum_{i=1}^{k} \sum_{c=1}^{M} a_{i}^{c} \quad \forall i \varepsilon K, c \varepsilon M
$$

subject to:

$$
\begin{gathered}
\sum_{c=1}^{M} a_{i}^{c} \leq 1 \quad \forall \quad i \varepsilon K \\
\sum_{i=1}^{K} a_{i}^{c} \leq 1 \quad \forall \quad m \varepsilon M \\
P_{\min }-\left(\sum_{i=1}^{K} P_{j}^{m}-\sum_{i=1}^{K} G_{i, m}+N_{o}\right)-\sum_{i=1}^{K} \delta_{i}^{m} \geq T-\sum_{i=1}^{K} a_{i}^{m} \delta_{i}^{m} \\
P_{i}^{c}-G_{i i}-\left(\left(\sum_{j=1, j \neq i}^{k} P_{j}^{c}-\sum_{j=1, j \neq i}^{N} G_{j, i}\right)+P B S_{c}-G_{B S, i}+N_{o}\right)-\sum_{j=1, j \neq i}^{K} \lambda_{j}^{c} \geq T^{\prime}-\sum_{j=1, j \neq i}^{K} a_{j}^{c} \lambda_{j}^{c} \\
P_{i}^{c} \leq a_{i}^{c} . P_{\max }
\end{gathered}
$$

where

$$
\begin{gathered}
\lambda_{j}^{c}=G_{j, i} \\
\delta_{i}^{m}=-G_{i, m}
\end{gathered}
$$


Here, the optimization variables include the $a_{j}^{c}$ and $P_{j}^{c}$. The binary assignment variables are responsible for identifying the specific CR pairs, which can be admitted without violating PU constraints. Corresponding to the assignment variables $\left(a_{j}^{c}\right)$, the power variables $\left(P_{j}^{c}\right)$ represent the specified transmission power for the admitted CR pairs [39].

This problem belongs to the class of Mixed Integer Linear Programming (MILP) and is NP hard. Optimal solution to this problem can be obtained using branch and bound technique. However, complexity of the branch and bound algorithms is exponential, and are not suitable for large scale implementation.

\subsubsection{Maximization of Admitted Users based on Hungarian Algo- rithm}

Here, we introduce a novel method based on Hungarian Algorithm to solve this problem optimally in a centralized manner. The proposed algorithm transforms the original NP hard problem to an instance of minimum weighted bipartite matching. The complexity of the proposed algorithm is strictly polynomial, and is upper bounded by $O\left(n^{3}\right)$. A Hungarian algorithm based method for maximization of admitted CR users in overlay scenarios is presented in [39]. However, this method relies on exploitation of temporal spectrum holes only, by allowing CR users to operate on idle PU bands and therefore is not suitable for our system setup. Even though we derive our motivation from [39], there exist several key differences in both these works.

Our proposed algorithm has three key steps, as described below:

Step 1: Minimum Power Calculation - Since our aim to is to admit CR users at rates no larger than the minimum specified rate it seems logical to assign all admitted $\mathrm{CR}$ users the minimum required transmission power. We start by finding the minimum transmission power required to achieve this rate for all CR pairs corresponding to the available PU channels.

$$
P_{i, m}^{m i n}=T^{\prime}+10 . n \cdot \log _{10}\left(d_{i}\right)+P B S_{c}-10 . n \cdot \log _{10}\left(d_{B S, i}\right)-N_{o} \quad \forall i \varepsilon K, m \varepsilon M
$$

Step 2: Primary Receiver Protection Check - Having obtained the minimum required transmission powers from step 1, we proceed to check if CR transmissions at these transmission powers leads to harmful interference at PRs. This is done by estimating the perceived SIR at PRs:

$$
S I R_{i, m}^{P R}=P_{\min }-\left(P_{i, m}^{\min }-10 . n \cdot \log _{10}(d)+N_{o}\right) \quad \forall i \varepsilon K, m \varepsilon M
$$

As discussed earlier we will consider three cases:FLA,PLA and NLA for our evaluation. Our performance metrics will be average network throughput and number of admitted users. 
Step 3: Weight assignment - Based on the obtained estimated SIR at PRs from step 2, we assign weights to the edges as:

$$
w_{i, j}=\left\{\begin{array}{l}
P_{i, m}^{m i n} \quad \text { if } \quad S I R_{i, j} \geq S I R_{T} \\
\infty \quad \text { if } \quad \operatorname{SIR}_{i, j} \prec \operatorname{SIR}_{T}
\end{array}\right.
$$

At the end of Step 3 we have a weighted graph $G$ with a weight matrix $W$ and consisting of two bipartite sets $U$ and $V$, with $U$ representing the set of CR users and $V$ representing the set of available channels, as shown in figure.

The elements of the weight matrix $W$ represent the edge weights, which are proportional to transmission power. An edge weight of infinity for a given CR pair on a specific $\mathrm{PU}$ channel implies that the CR user is prohibited to transmit using the that specific PU band. Often, it may be possible to have unequal number of elements in the two bipartite sets $U$ and $V$, thus leading to an unbalanced bipartite graph. In order to make the original bipartite graph balanced we introduce dummy variables/nodes on either side, depending on whether the number of CR pairs is greater than number of available PU channel or vice versa 5.1, 5.2. For a given set $(U / V)$, for each of the inserted dummy nodes we also introduce dummy edges, which connect them to all the vertices of the opposite set $(V / U)$. These dummy edges are assigned an edge weight of infinity.

Running the Hungarian Algorithm on the resultant bipartite graph will return a perfect matching which will both be maximal and maximum. Thus implying that the proposed algorithm admits maximum number of $\mathrm{CR}$ users, given system constraints. The resultant binary assignment matrix $A$, whose elements $a_{i, j}$, depict the usage of PU band $j$ by CR pair $i$ if $a_{i, j}=1$. For a given element of $A$, if $a_{i, j}=0$ the CR pair $i$ is prohibited from using the PU band $j$.

The mathematical formulation corresponding to the bipartite graph constructed can be given as follows:

$$
\operatorname{Minimize} \sum_{i=1}^{k} \sum_{c=1}^{M} P_{i}^{c} \cdot a_{i}^{c} \quad \forall i \varepsilon K, c \varepsilon M
$$

subject to:

$$
\begin{array}{ll}
\sum_{c=1}^{M} a_{i}^{c}=1 & \forall \quad i \varepsilon K \\
\sum_{i=1}^{K} a_{i}^{c}=1 \quad \forall & m \varepsilon M
\end{array}
$$

Let $L$ and 1 denote the linear constraint matrix and identity vector formed on left hand side and right hand side respectively. Further, let $x$ denote the vector of assignment variables. Then the set of constraints (5.23) and (5.24) can be concisely represented as: 


$$
\text { L.x } x^{T}=1
$$

The following theorem suggests that the all the vertices of the polytope defined by the LP are integral, and therefore correspond to perfect matching of $G$.

Theorem 1: Let $\mathbf{A}$ be an integer matrix. If $\mathbf{A}$ is totally unimodular, then the vertices of the polytope $\left(x: A x^{T}=b^{T}, x \geq 0\right)$ are integral whenever $\mathbf{b}$ is integral [35].

Where a Unimodular matrix is defined as:

Definition 1: A matrix $L$ is said to be totally unimodular if the determinant of every square submatrix (minor) of $L$ lies in the set $(-1,0,1)$ [39],[35]. For our LP, the matrix $L$ can be proven to be unimodular [39], [35].

\subsection{Numerical Results}

In this section we will evaluate the performance of our resource management strategies under different levels of location awareness: FLA, PLA and NLA, along with analysis of impact of system parameters (such as No of Users, SIR threshold, duty cycle etc) and propagation parameters $(n)$ on CR network performance. We will consider both SRM and MAU separately.

\subsubsection{Simulation Setup}

We consider a setup where the CR network coexists with a PN. The PN consists of a circular cell area of radius $R_{i}$, with $\mathrm{BS}$ at the center and $N$ PRs distributed uniformly within its coverage.

$K \mathrm{CR}$ pairs comprising of a $\mathrm{CR}$ transmitter and $\mathrm{CR}$ receiver are also located within the area. The CR receiver is at a distance $d$ form the CR transmitter. Locations of $\mathrm{CR}$ transmitters are generated randomly within the coverage area of the PN. The PN is equipped with ATPC to ensure a minimum received power $\left(P_{\min }\right)$ at the receivers. The PRs and CR pairs have SIR thresholds given by $S I R_{P U}$ and $S I R_{C R}$ respectively.

\subsection{Numerical Results for Sum Rate Maximization}

In this section we provide simulation results concerning SRM, and analyze impact of system and environment parameters on CR network performance. 


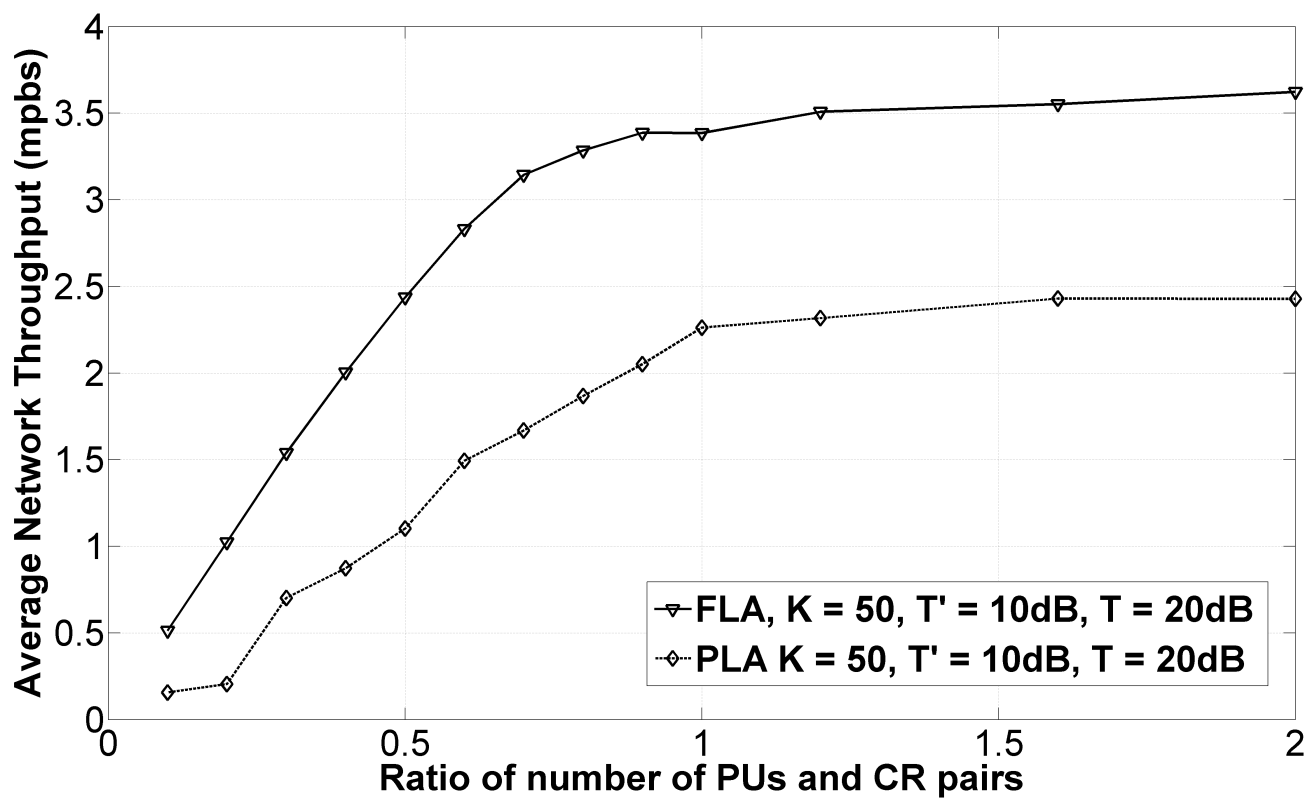

Figure 5.3 - Effect of Density of Users on Average Network Throughput

\subsubsection{Effect of Number of Users}

Figures 5.3 and 5.4 show the impact of number of users (PU and SU) on the average network throughput of the CR network and number of admitted CR users respectively. They clearly depict the relationship between the number of CR pairs and PU bands for both the PLA and FLA scenarios. Following are the simulation parameters for this scenario:

1. No of CR Users $(K)=50$

2. SIR threshold for $P U=20 \mathrm{~dB}$

3. SIR threshold for $\mathrm{SU}=10 \mathrm{~dB}$

4. Path loss Exponent $(n)=4$

5. Ambient Noise $=-90 \mathrm{dBm}$

6. Minimum Received Power at $\mathrm{BS}=-60 \mathrm{dBm}$

Since each PR is assigned exactly one unique band, more PU bands implies more PRs and vice versa. According to 5.3, when the ratio of number of PUs to number of CRs is less than one, the FLA CRN outperforms the PLA CRN. This implies that the 


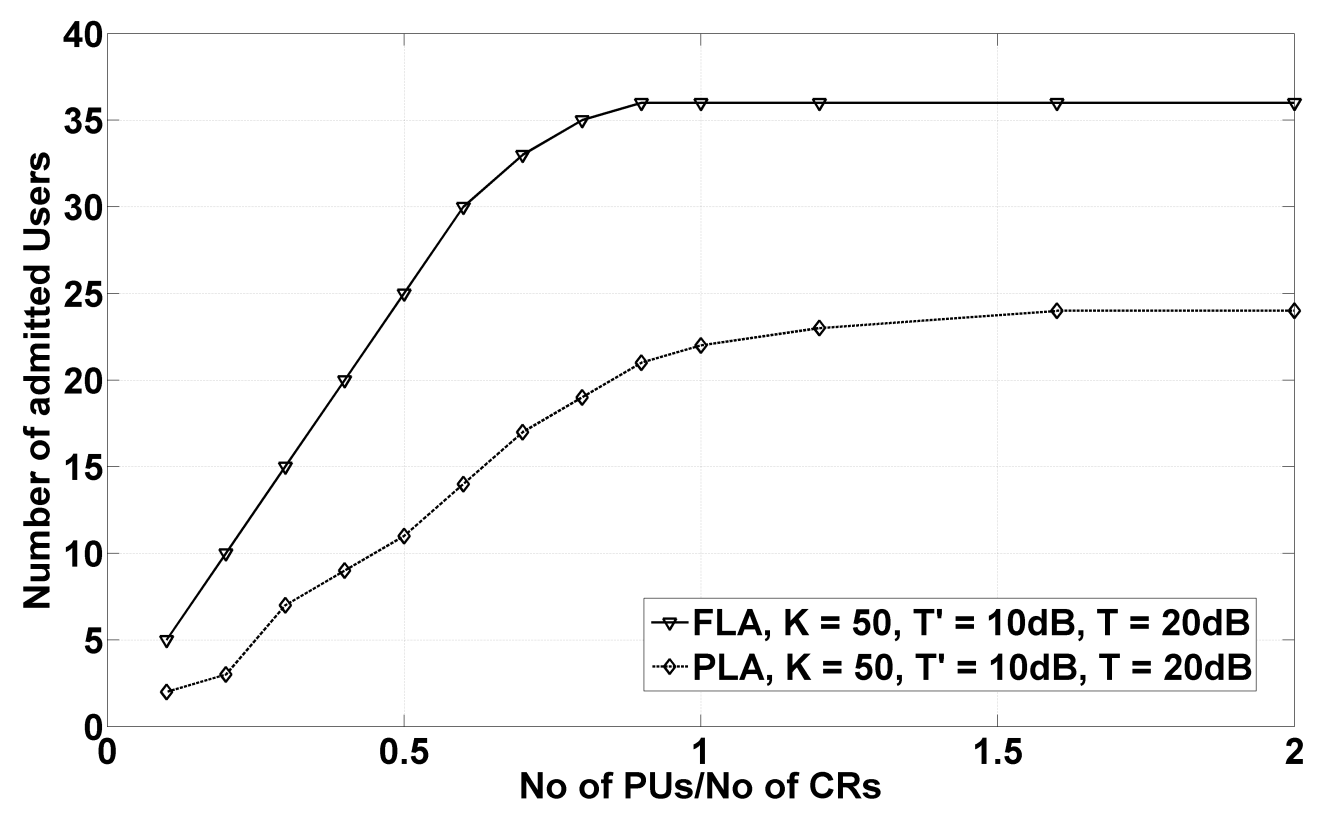

Figure 5.4 - Effect of User Density of Number of Admitted Users

FLA CRN is able to utilize spatial spectrum opportunities more efficiently as compared to PLA CRN. The gap in performance widens gradually till the ratio of CR and PU users equals one, beyond which it becomes constant. At this point no further $\mathrm{CR}$ requests can be accommodated given the current interference constraints. The 5.4 shows a similar trend where there is a consistent performance gap between the FLA and PLA scenarios.

The FLA CRN is able to cater to far more CR requests as compared to PLA CRN. As will be seen in the later figures, the performance gap between PLA and FLA scenarios depends on other system parameters as well.

\subsubsection{Effect of distance between CR pairs}

Figures 5.5 and 5.6 depict the impact of distance between CR pairs $\left(d_{o}\right)$ i.e., distance between $C R_{T x}$ and $C R_{R x}$ on system performance of the overlaid CR network. Specifically, figure 5.5 shows the impact of $d_{o}$ on the average network throughput of the CRN. In order to magnify the performance gap, the radius of both the PN and CRN is kept at $1000 \mathrm{mt}$. Here, it can been seen that the average network throughput of the CRN is significantly high for scenarios where $d_{o}$ is less than $100 \mathrm{mt}$. This is because CR pairs with short separation experience higher SINR ratios, which results in increase of network throughput Also, the performance gap between PLA and FLA scenarios is very less for $d_{o}$ less than $100 \mathrm{mt}$, implying that full location awareness for such scenarios is 


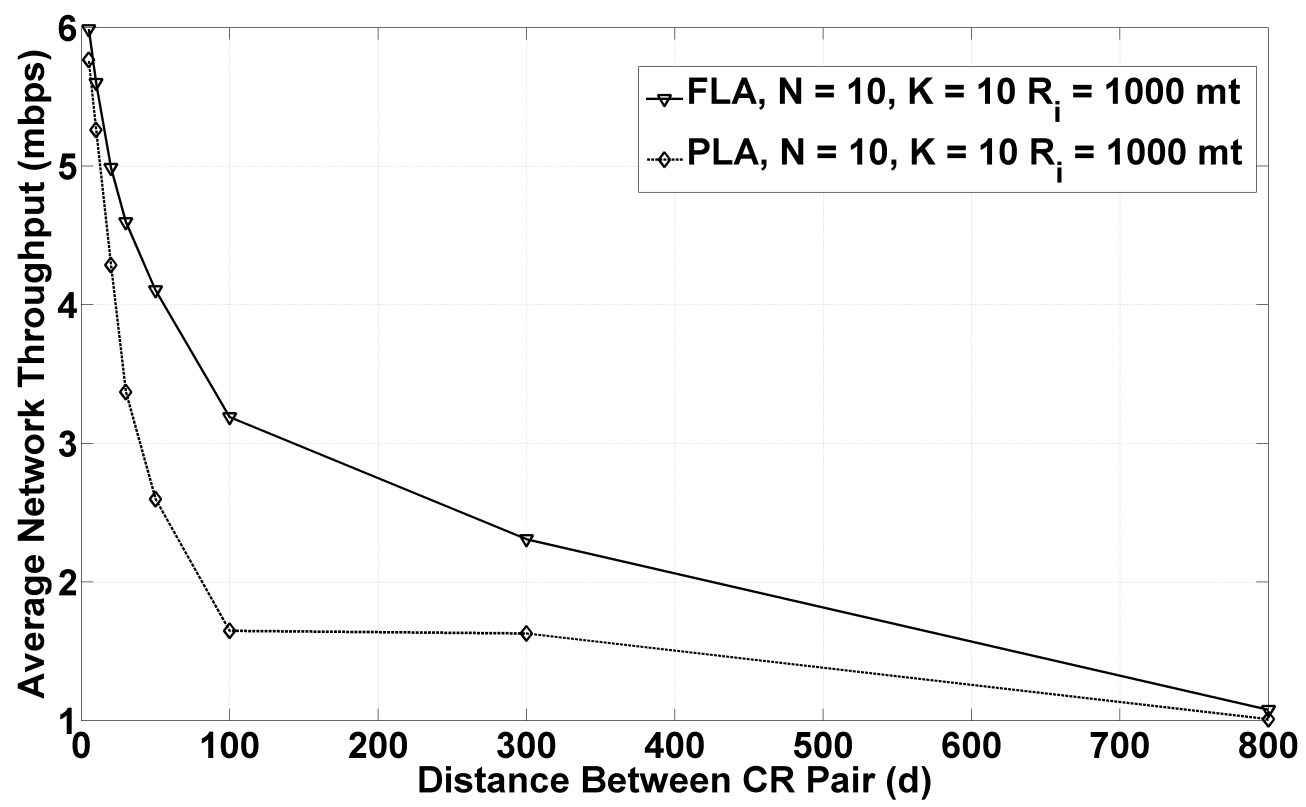

Figure 5.5 - Effect of Distance between Cr pairs on Average Network Throughput

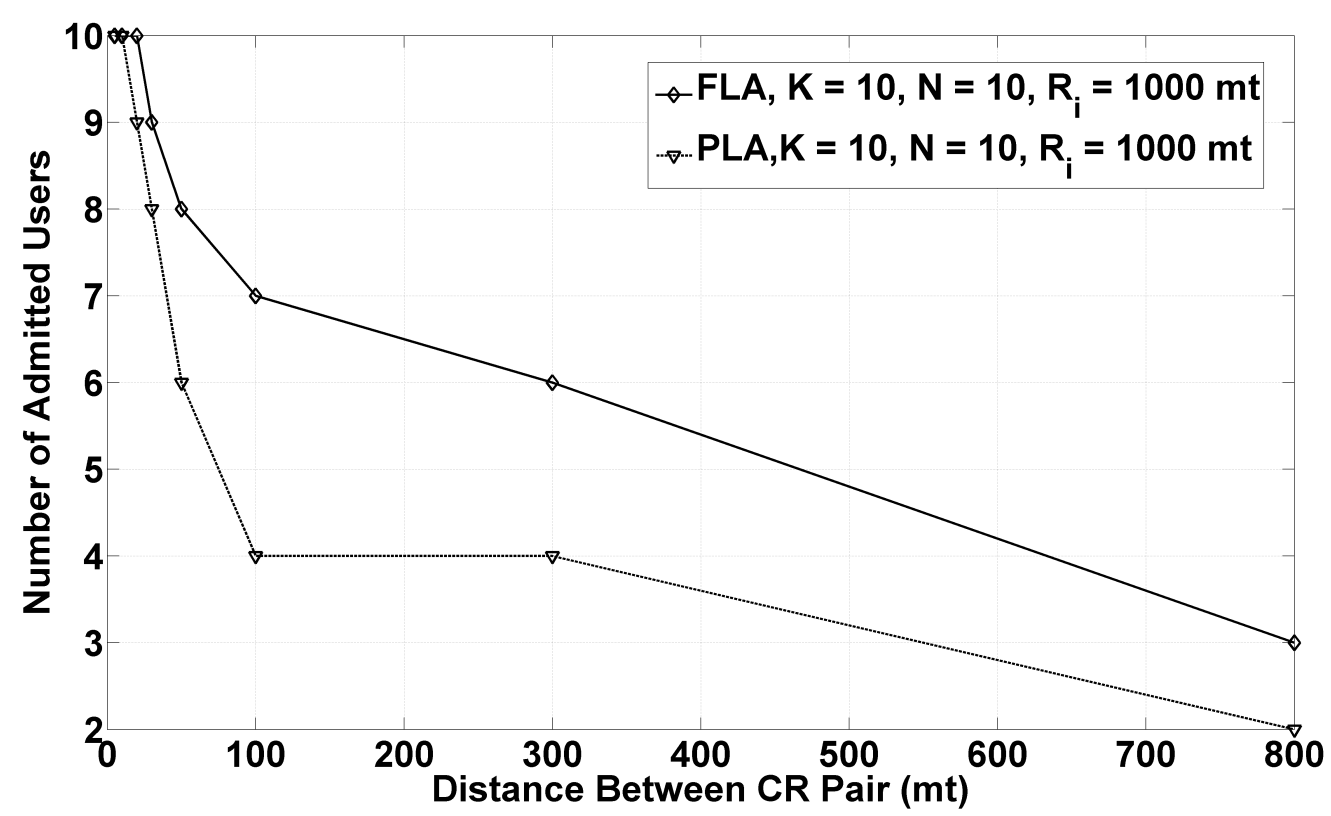

Figure 5.6 - Effect on Distance between CR pairs on Number of Admitted Users 


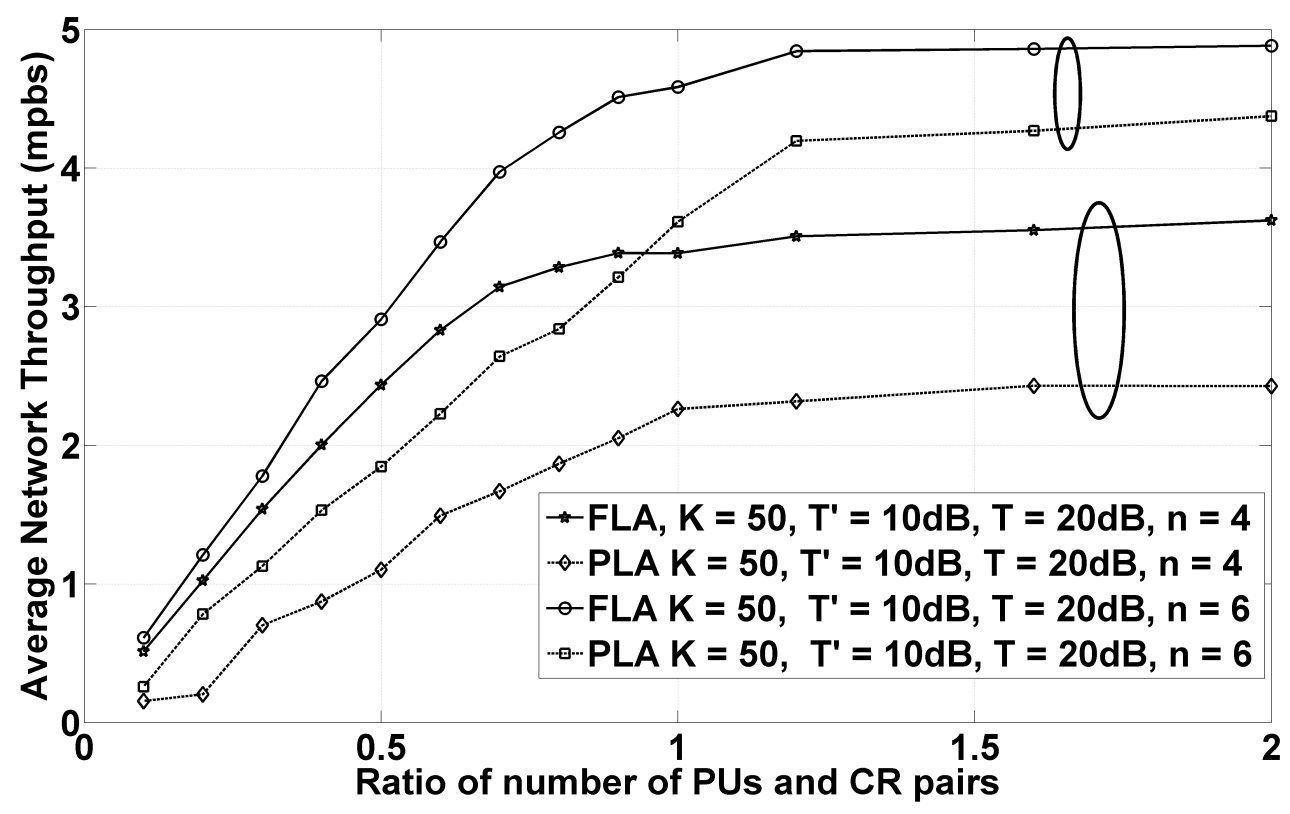

Figure 5.7 - Effect of Propagation Environment on Average Network Throughput of CR Network

not significantly beneficial.

A similar trend can be observed in figure 5.6, where number of admitted users is comparable for scenarios with $d_{o}$ less than $100 \mathrm{mt}$. However, as $d_{o}$ increases the performance gap widens implying that full location leads to better system performance as can be seen in figures 5.5 and 5.6. When $d_{o}$ approaches large values $(\geq 300 \mathrm{mt})$ the performance gap reduces, as chances of accommodating CR requests for these scenarios become smaller, owing to the higher $\mathrm{CR}$ transmission power requirements which results in harmful interference at PRs 5.55.6.

\subsubsection{Effect of Propagation Environment}

The received signal strength (RSS) at PRs due to CR transmissions depends significantly on the nature of propagation environment. For scenarios where the signal attenuation is severe, the perceived signal strength from CRs will be much lower compared to scenarios where signal attenuation is mild.

Figures 5.7 and 5.8 show the impact of path loss exponent $(n)$ on system performance in terms of the average network throughput and number of admitted users respectively. Here, for the sake of comparison two cases are concerned. For the first case $n$ is considered to be 4 , while for case $2 n$ is assumed to be 6 . Clearly for both the 5.7 


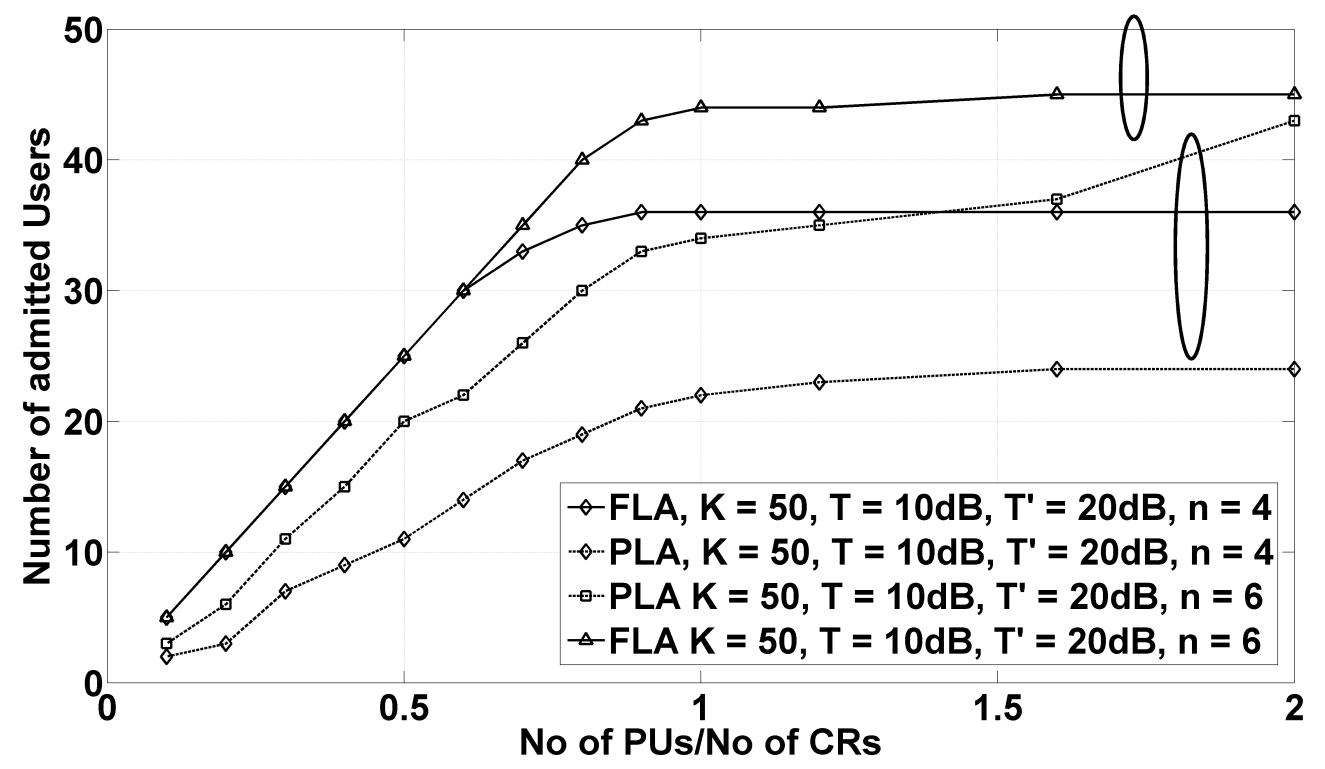

Figure 5.8 - Effect of Propagation Environment on Number of Admitted Users

and 5.8 the performance gap between FLA and PLA scenarios decreases with increase in $n$. Also, increase in $n$ leads to increase in average network throughput and number of admitted users.

This implies that for scenarios such as under water, indoor or basements, where propagation environment is harsh both FLA and PLA CRNs offer comparable performance. This result can be highly useful, as it can be used to guide future CR system design.

\subsubsection{Effect of Minimum Rate}

Minimum required rates for both PUs and CR users has significant impact on system performance of the overlaid CR network, as shown by figures 5.9 and 5.10 . Here, for the sake of comparison we have two cases. For case 1 the $S I R_{C R}=10 \mathrm{~dB}$ and $S I R_{P U}=$ $20 \mathrm{~dB}$, and for case $2 S I R_{C R}=3 \mathrm{~dB}$ and $S I R_{P U}=10 \mathrm{~dB}$.

As can be clearly seen, decrease in rate requirements leads to better performance. This can be explained as follows with regard to the Hungarian Algorithm based SRM presented in section XX: the PU rate requirements are responsible for controlling the transmission power of the CRs, whereas the SIR requirements of the CRs is responsible for controlling the number of admitted CR users. Decrease in SIR for PUs leads to increase in transmission power of CRs which increases their SIR which leads to increase 


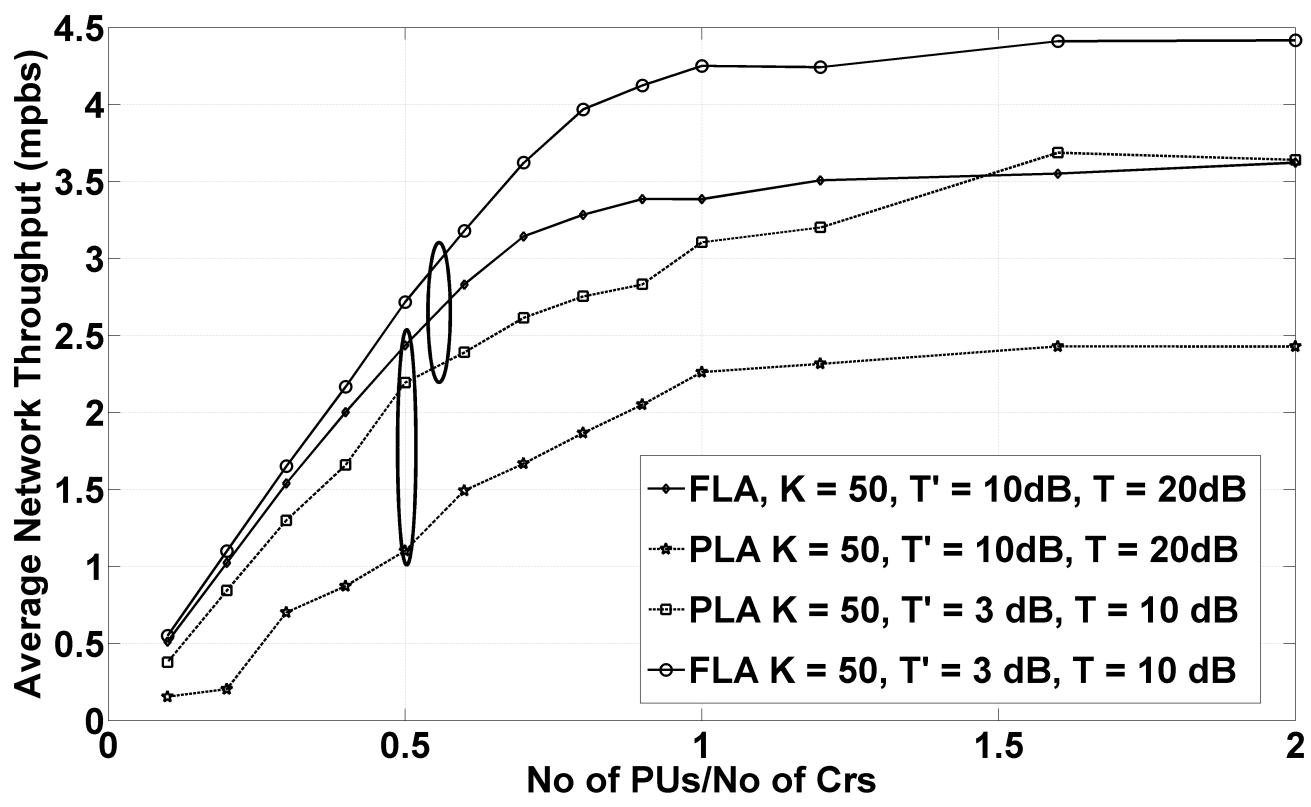

Figure 5.9 - Effect of Minimum SIR Thresholds on Average Network Throughput

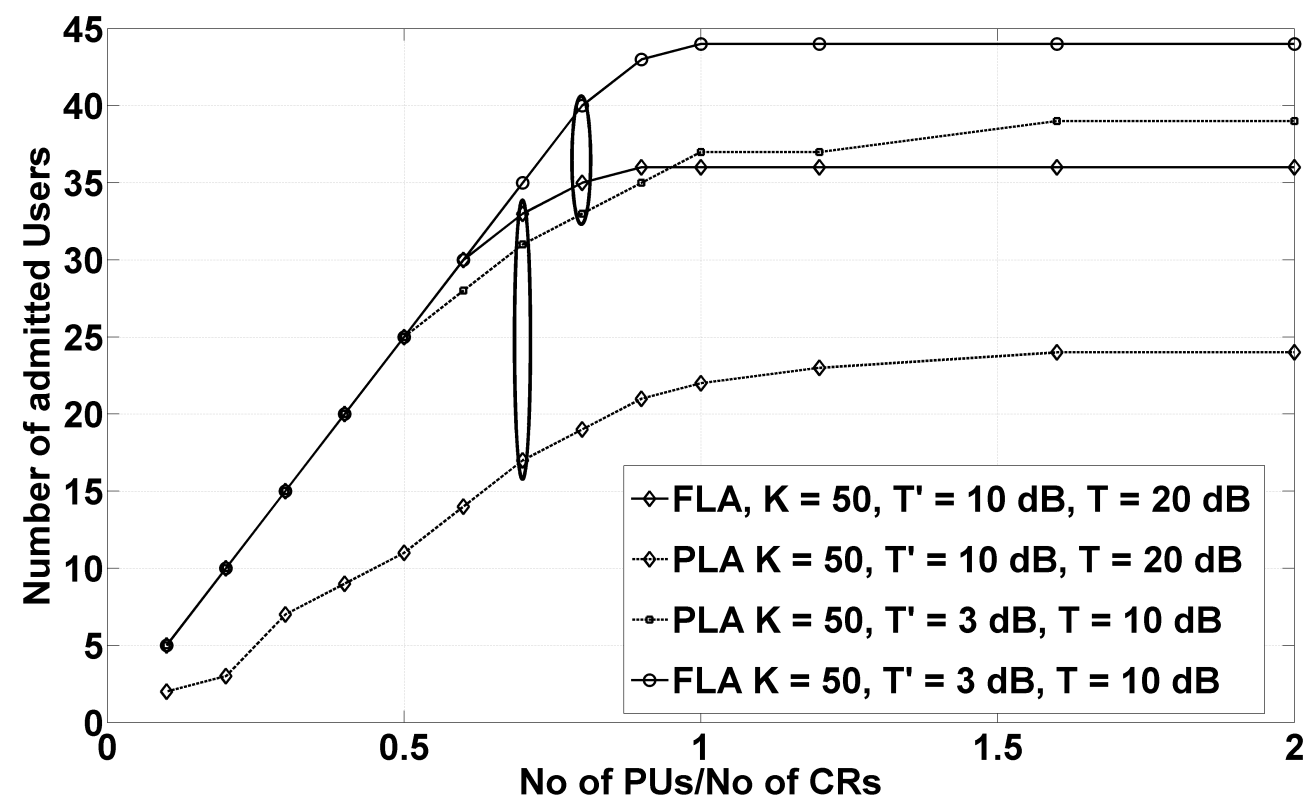

Figure 5.10 - Effect of SIR Thresholds on Number of Admitted CR Users 


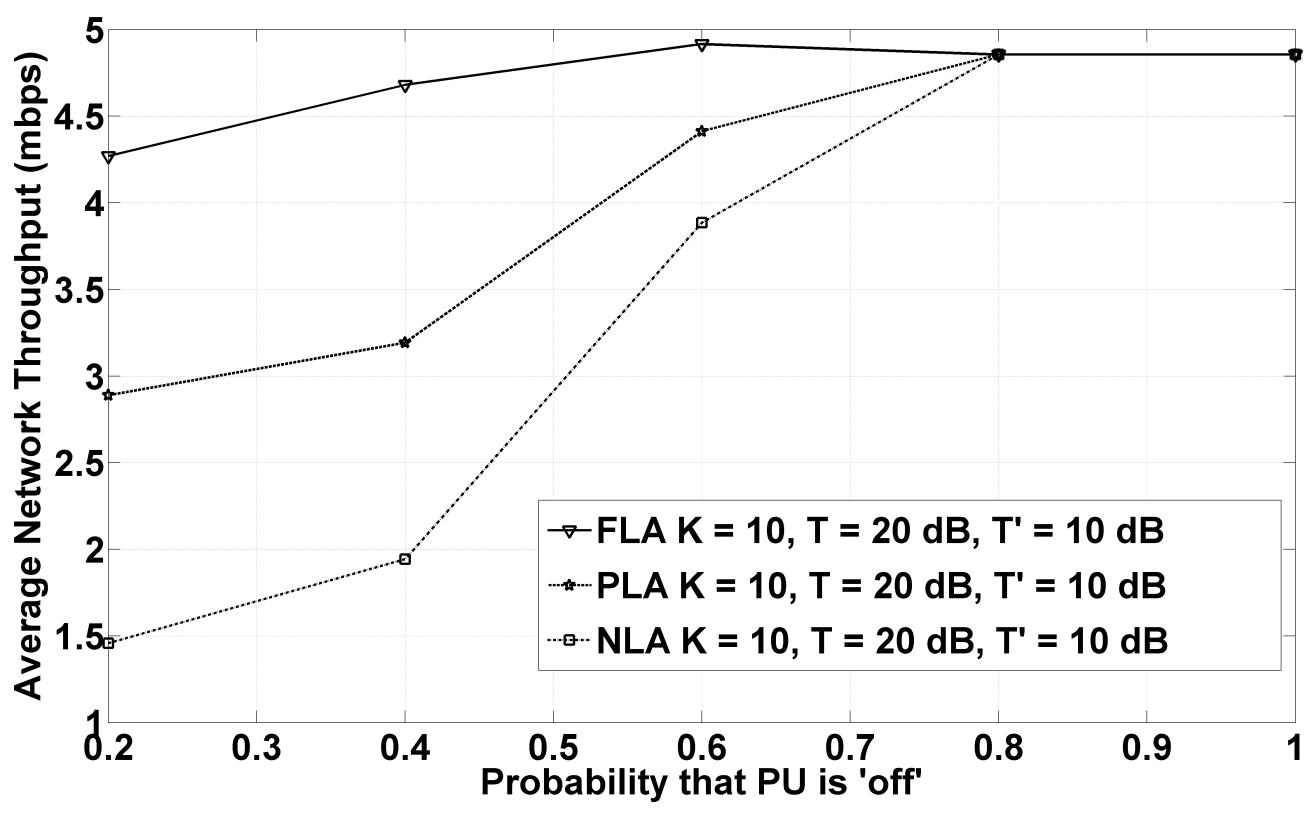

Figure 5.11 - Effect of PU Activity on Average Network Throughput of the CR Network

in average network throughput, as evident in figure 5.9. On the other hand decrease in SIR requirement for CRs leads to increase in the number of admitted users as can be clearly seen in figure 5.10 .

Based on our observations concerning minimum rate requirements it can inferred that if our aim is to maximize the number of admitted users, the rate requirements of CR users is the deciding factor. Whereas if our aim to maximize network throughput, the PUs need to be less sensitive to interference i.e., low SIR requirements. This observation can be useful in formulating the frequency selection strategy for CR nodes, if CR nodes seek to attain high data rates they should target bands which are less susceptible to interference i., have low SIR requirements for users licensed to use that particular band.

\subsubsection{Effect of Duty Cycle}

Till now we have examined the impact of location awareness on system performance of overlaid CR network, which aims at reusing occupied primary frequency bands, to establish single hop links within its users. Specifically, we have focused our attention on utilization of spatial spectrum holes. Here, we have assumed that PUs are always active i.e., PRs are always transmitting from BS. However, in practice all PU bands can be characterized using duty cycle. The duty cycle of a PU depicts the percentage of time the PU is active. 


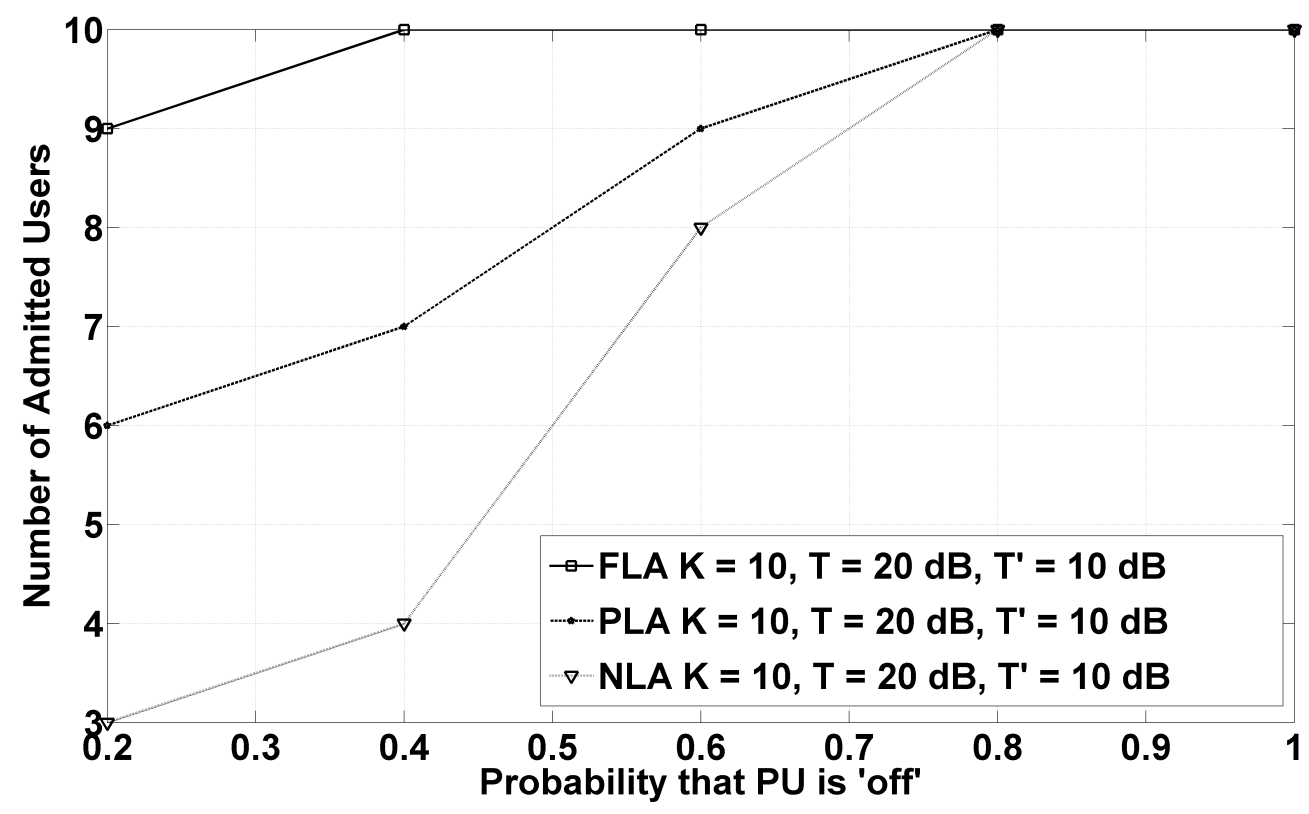

Figure 5.12 - Effect of PU Activity on Number of Admitted CR Users

In order to examine impact of location awareness in scenarios where the PUs have bursty transmissions i.e., PUs are not always transmitting, we examine three cases FLA, PLA and NLA. As pointed out earlier, both FLA and PLA are capable of using both spatial and temporal spectrum whereas NLA CRNs can only utilize temporal spectrum opportunities. Figures 5.11 and 5.12 depict the system performance for all the three cases.

Here the occupancy of PUs is modeled as a random variable, whose value is either zero or one with probability of one being dictated by duty cycle.

As can be seen from 5.11 and 5.12 all NLA, FLA and PLA perform equally efficient when probability of PU being "off" approaches unity. This is a crucial observation as it means that CR nodes operating in frequency band such as TV, which are vacant need not be location aware and thereby can save on cost. 


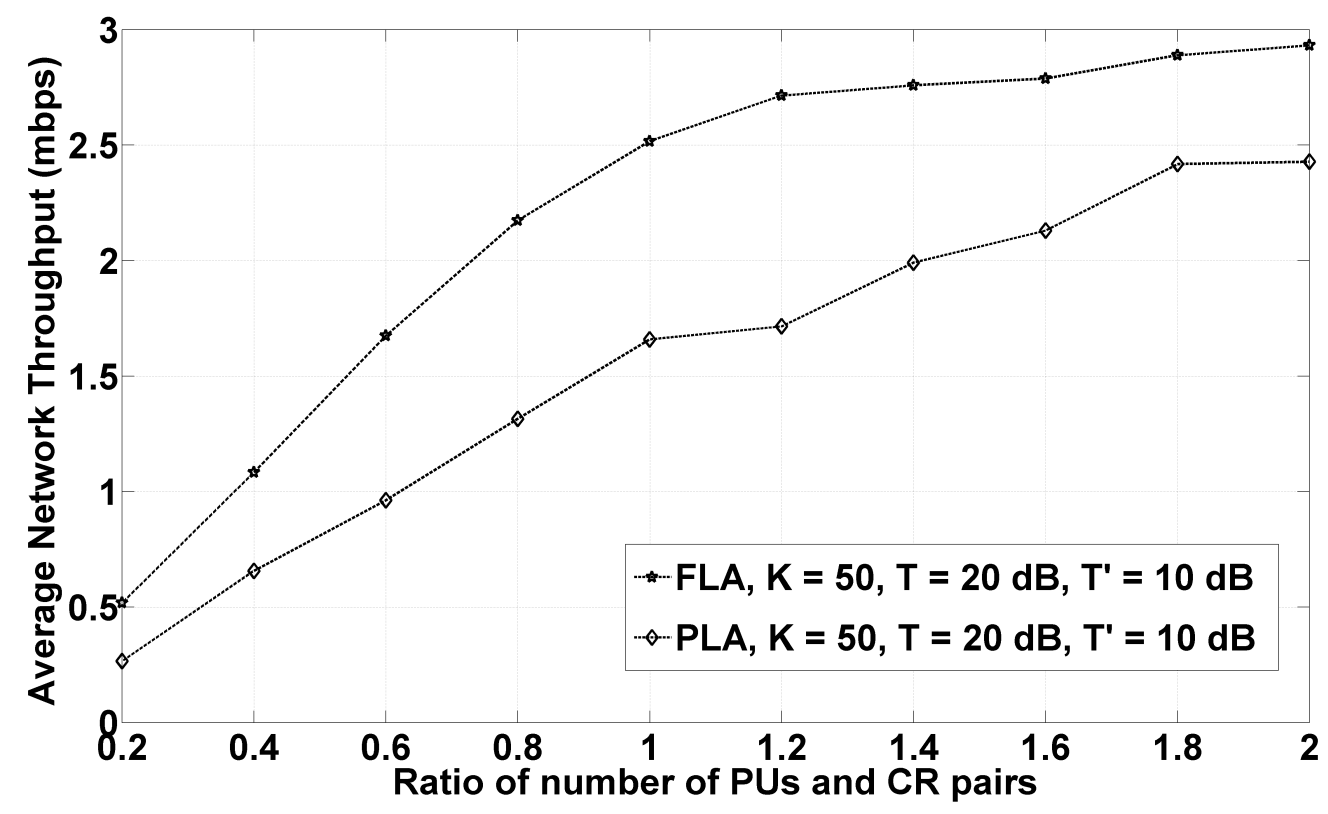

Figure 5.13 - Effect of Density of Users on Average CR Network Throughput.

\subsection{Numerical Results for Maximizing Number of Ad- mitted Users}

\subsubsection{Effect of Number of Users}

Here, the impact of location awareness on the relationship between number of CR pairs and PU bands for MAU under different scenarios is analyzed. Since each PR is assigned exactly one unique band, more PU bands implies more PRs and vice versa.

Figures 5.13 and 5.14 show the impact of location awareness on the CR network throughput and number of admitted CR users respectively, for differing levels of user density. On comparing figures 5.13 and 5.3, it can inferred that performance gap between PLA and FLA scenarios is more significant for Sum Rate Maximization. A similar trend regarding number of admitted CR users can be observed upon comparing figures 5.4 and 5.14. This can be useful result, as it suggests that impact of location awareness is more pronounced in scenarios where our objective is to admit maximum number of CR users at maximum possible transmission rate. Therefore, it might be suggested to have full location awareness for CR networks requiring superior performance, whereas CR users requesting a predefined minimum capacity could be made partially location aware. 


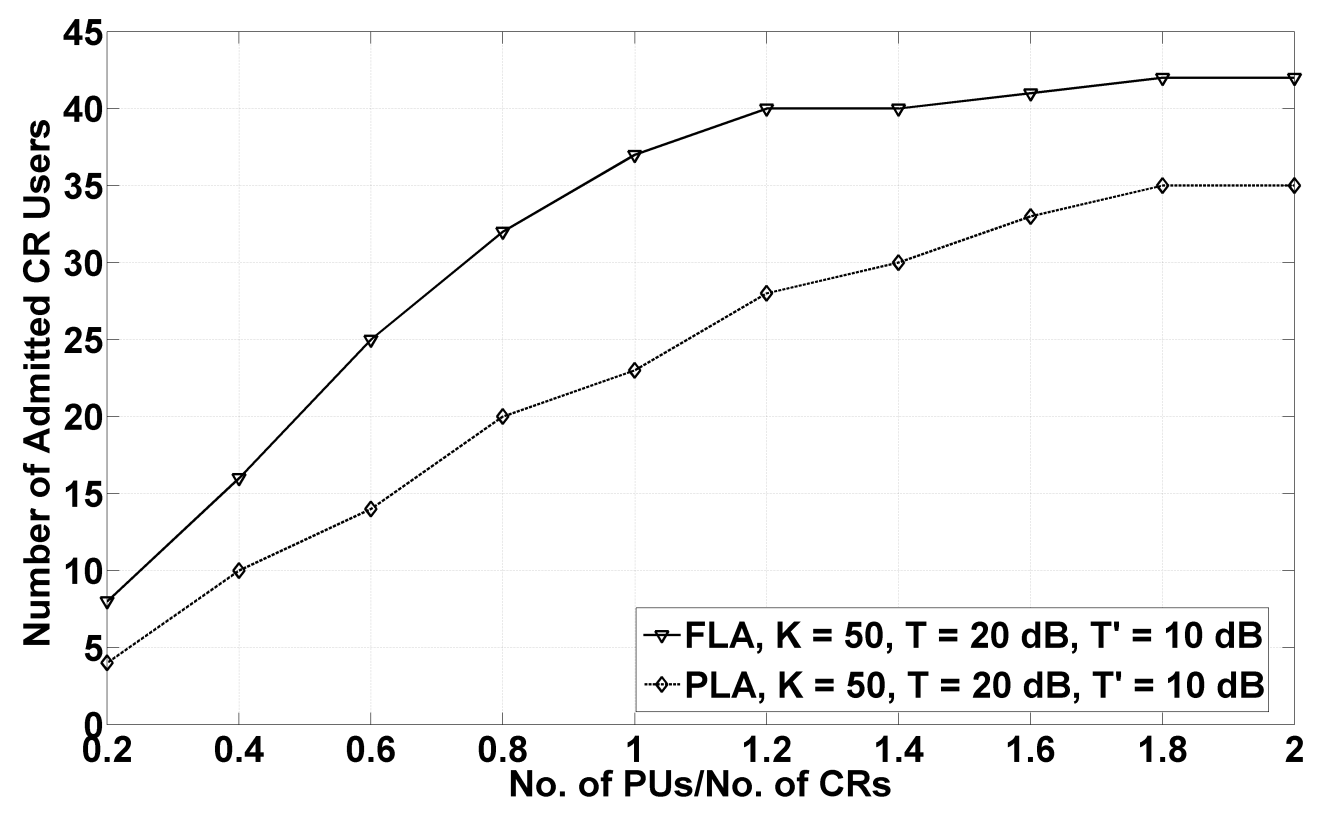

Figure 5.14 - Effect of Density of Users on Number of Admitted CR Users.

\subsubsection{Effect of distance between CR pairs}

Figures 5.15 and 5.16 depict the impact of location awareness on CR network throughput and number of admitted CR users, for different values of separation between the $C R_{T x}$ and $C R_{R x}$. The distance of separation is seen to impact the difference in performance of the CR network for both FLA and PLA significantly, with impact being more pronounced for larger values of separation. This observation implies that for short range communication (where distance between transmitter and receiver is less than $20 \mathrm{mt}$ ) both FLA and PLA scenarios perform almost equally, thus implying that full location awareness does not offer substantial performance gains for short range communication. As the distance of separation increases the CR network performance degrades as it becomes impossible to accommodate such CR transmissions due to resulting interference at PRs.

\subsubsection{Effect of Propagation Environment}

Figures 5.17 and 5.18 show the impact of location awareness on CR network performance in terms of average network throughput and number of admitted users, for two kinds of propagation environments. For the first scenario we assume the path loss exponent $(n)$ to be equal to four whereas for the latter case we assume it to be six. As evident 


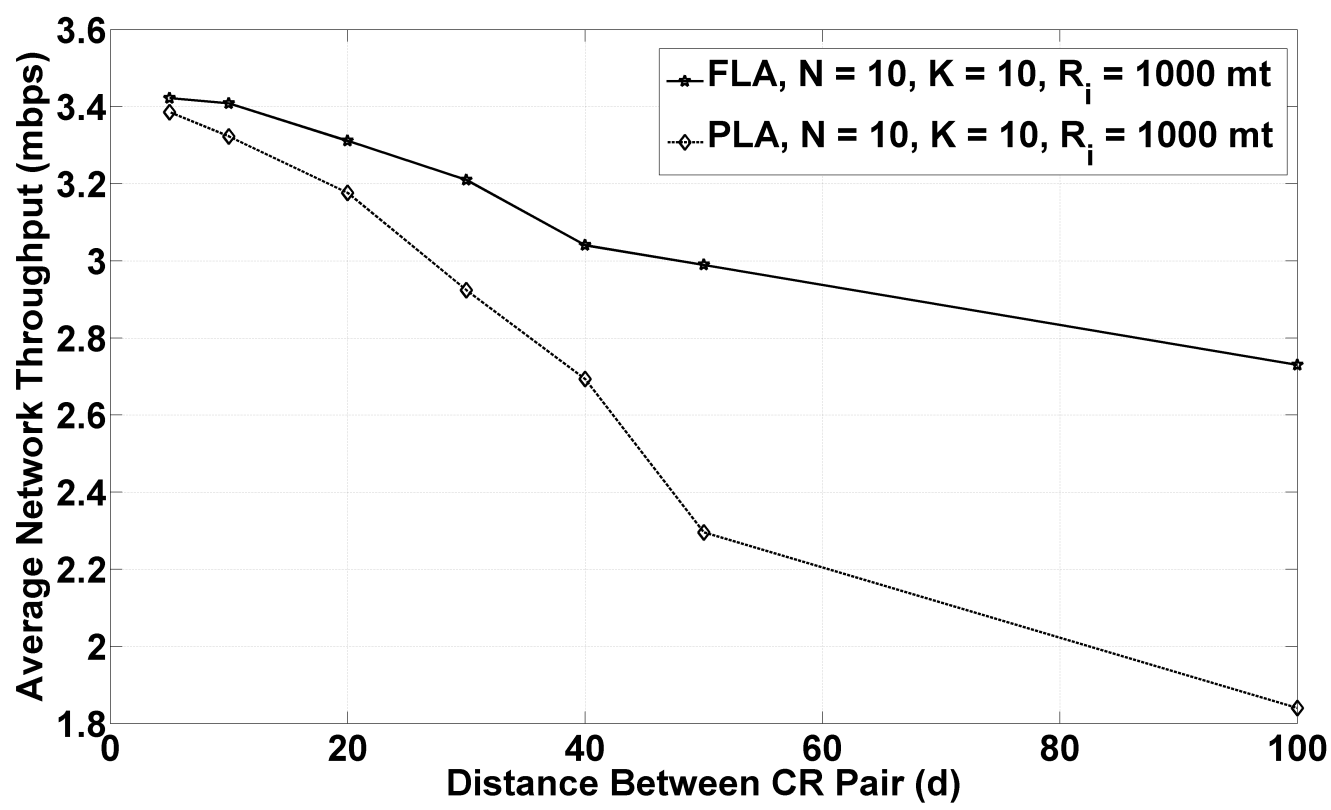

Figure 5.15 - Impact of Location Awareness on CR network throughput for differing levels of separation between $\mathrm{CR}$ transmitter and $\mathrm{CR}$ receiver.

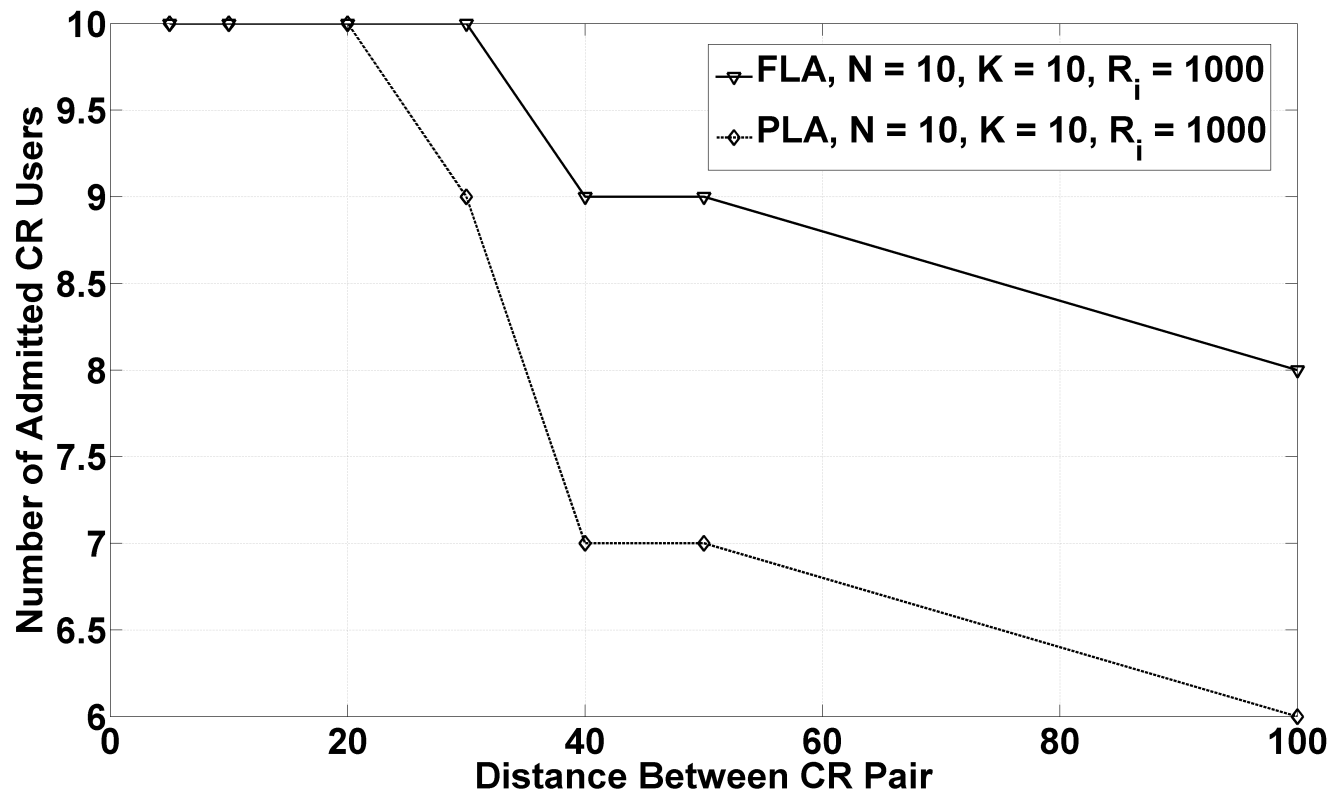

Figure 5.16 - Impact of Location Awareness on number of admitted CR users for differing levels of separation between $\mathrm{CR}$ transmitter and $\mathrm{CR}$ receiver 


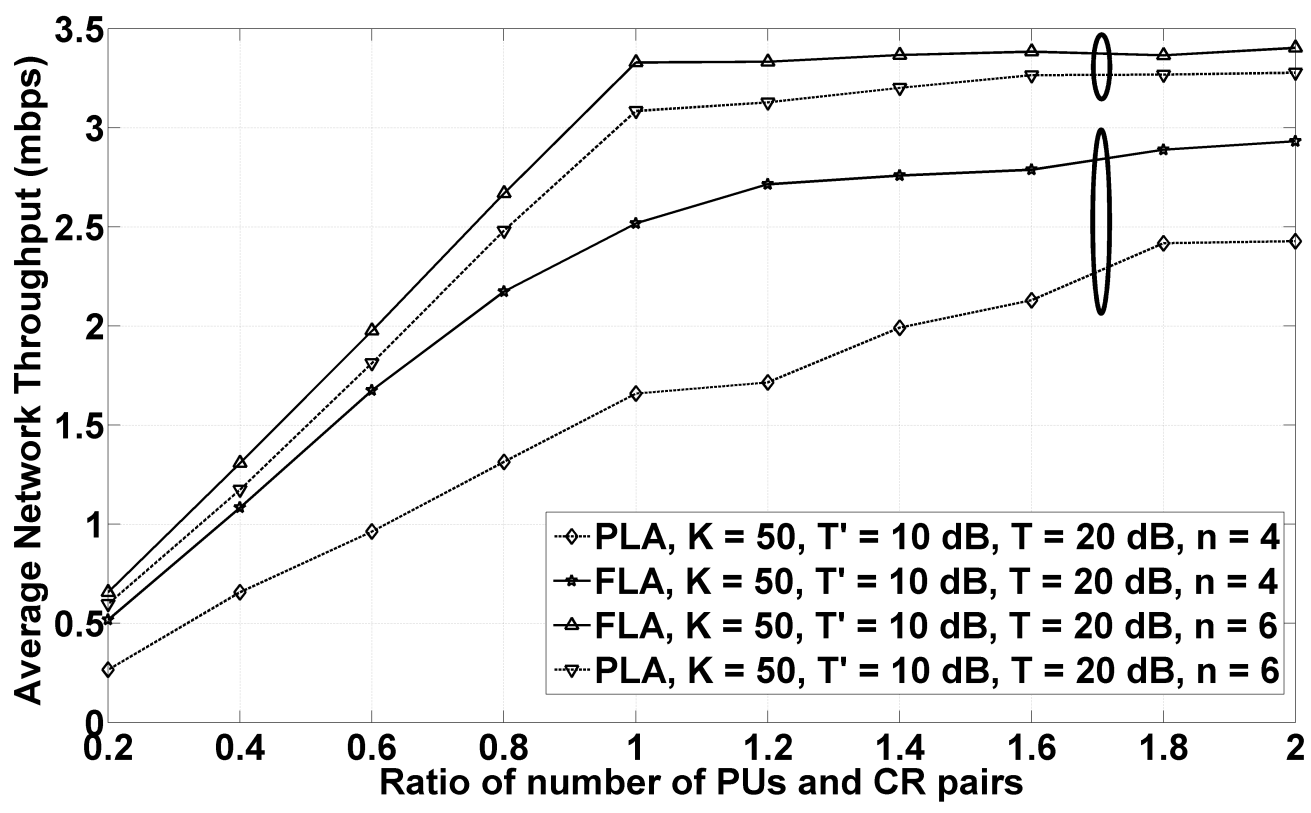

Figure 5.17 - Impact of nature of propagation environment on average CR network Throughput for PLA and FLA.

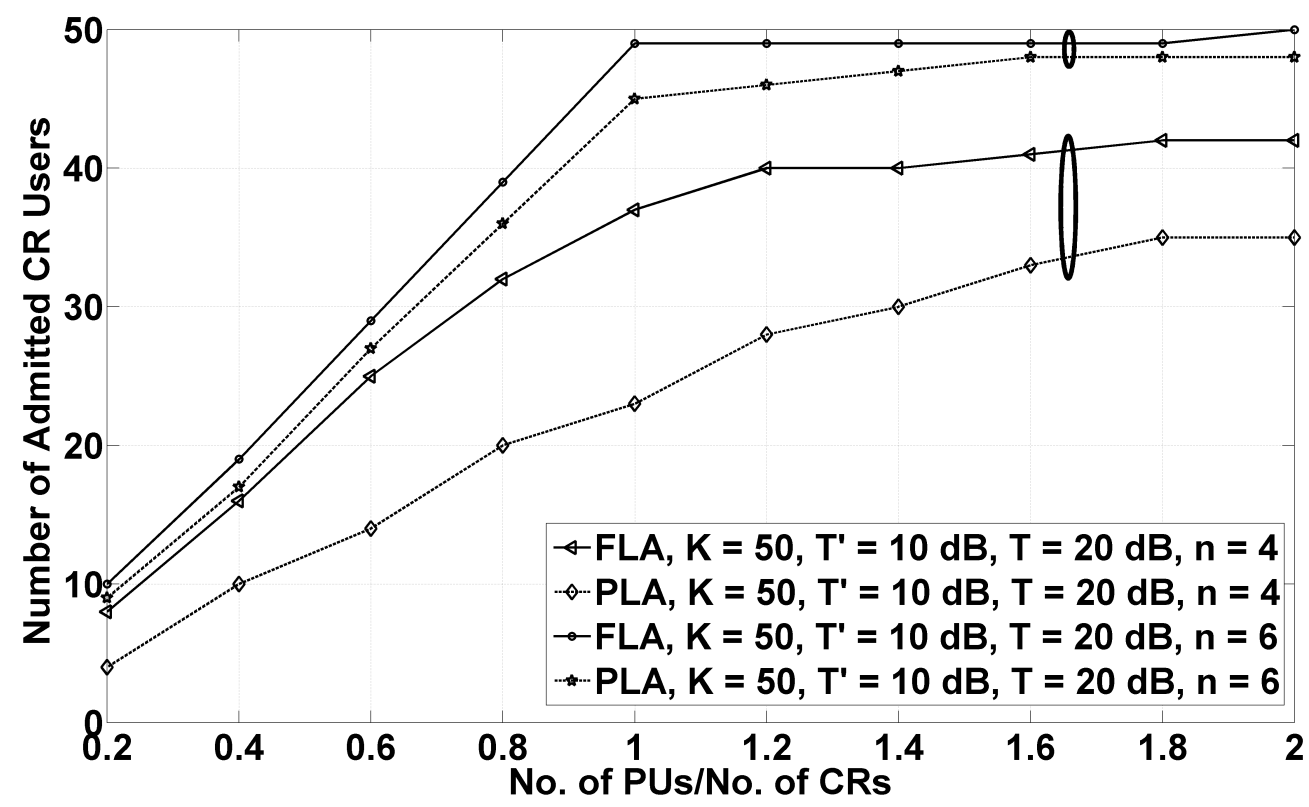

Figure 5.18 - Impact of nature of propagation environment on number of admitted CR users for PLA and FLA. 


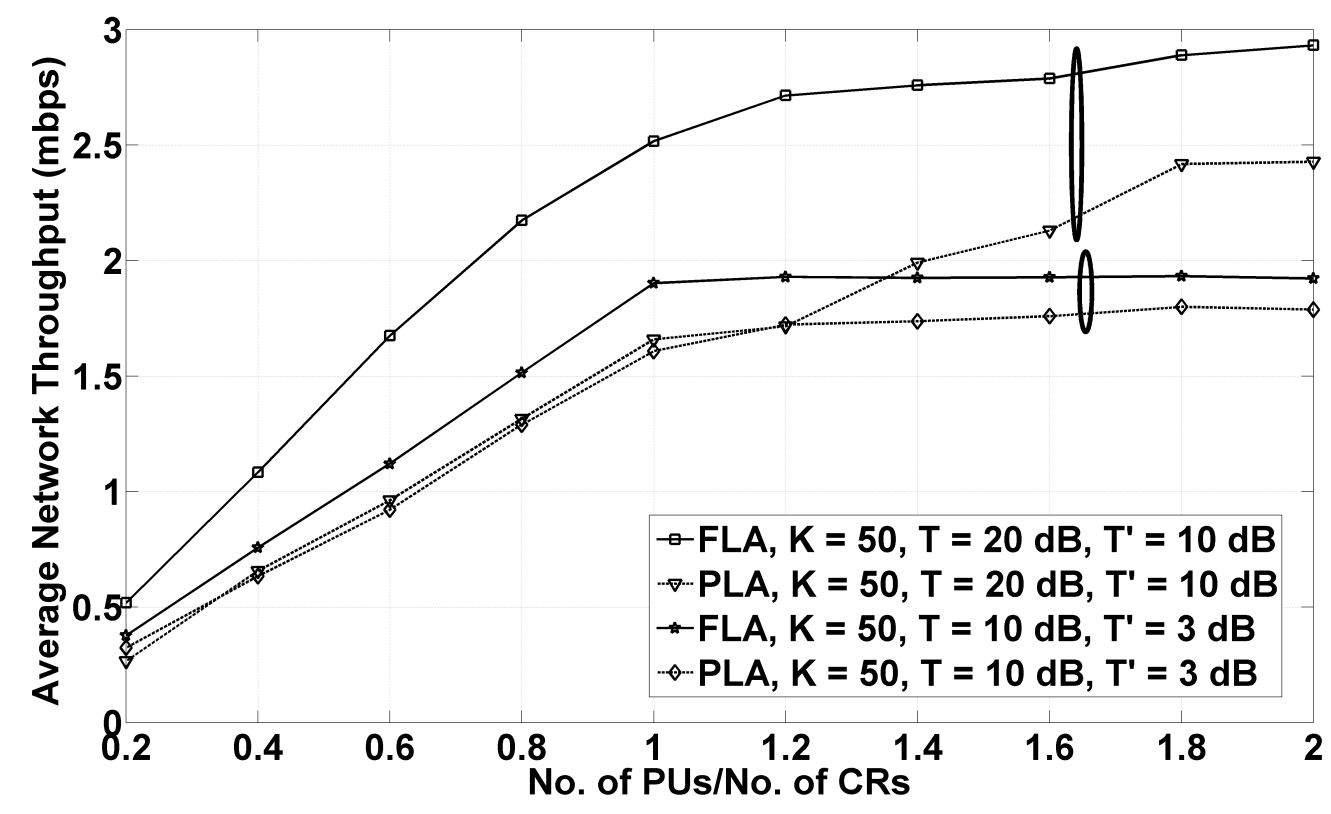

Figure 5.19 - Impact of SIR thresholds on CR network throughput for FLA and PLA.

there exists a strong relationship between the nature of propagation environment and the network performance of the overlaid CR network. For harsh propagation environments (higher $n$ ) the performance gap between FLA and PLA decreases, implying that full location awareness does not offer substantial gains in environments experiencing higher degrees of signal attenuation. An important implication of this result can help guide CR system design for indoor or underground applications, where signal attenuation is significantly high.

\subsubsection{Effect of Minimum Rate}

Figures 5.19 and 5.20 show the impact of decreasing SIR thresholds on CR network performance, for both FLA and PLA. As evident, decreasing SIR thresholds has significant impact on CR network performance. In contrast to SRM where decreasing $S I R_{P U}$ resulted in an increase in CR network throughput, the CR network throughput for MAU does not show a similar trend. This can be explained as follows: Since our aim during MAU is to admit CR users at rates no more than the predefined minimum, a decrease in $S_{I R}$ does not impact CR network throughput. The CR network throughput during MAU is independent of $S I R_{P U}$ as long as the perceived SIR at PUs is above the threshold, and is dependent solely on $S I R_{C R}$. Decrease in $S I R_{C R}$ results in a corresponding decrease in network throughput 5.20 . 


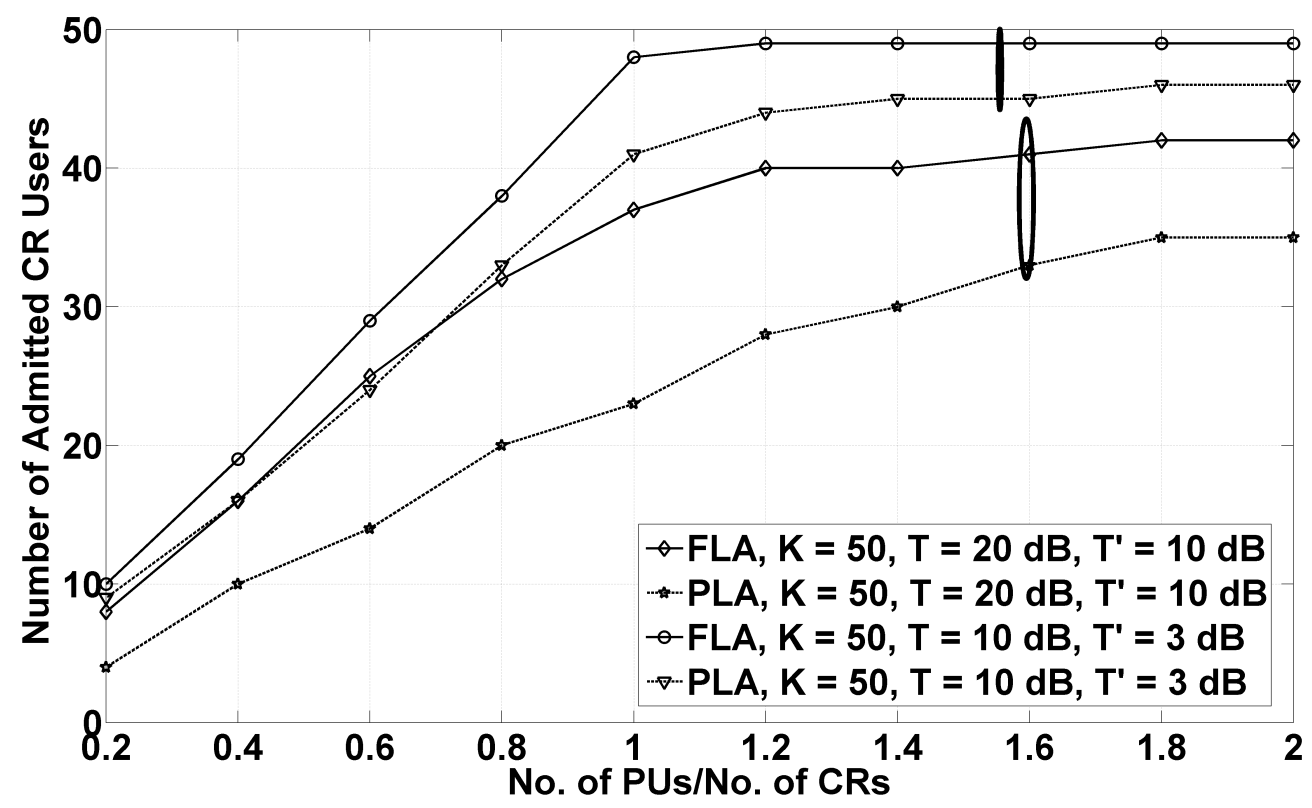

Figure 5.20 - Impact of SIR thresholds on number of admitted CR users for FLA and PLA.

However, decrease in $S I R_{P U}$ and $S I R_{C R}$ leads to an increase in number of admitted CR users 5.20. This follows from the fact that decrease in $S I R_{P U}$ implies that PUs are willing to tolerate more interference, thus admitting more CR users.

\subsubsection{Effect of Duty Cycle}

Figures 5.21 and 5.22 show the impact of PU duty cycle on CR network performance for varying levels of location awareness (FLA, PLA and NLA). As the probability of PU being off increases the performance gap between all scenarios (FLA, PLA and NLA) decreases. This is a useful result, as it implies that location awareness offers substantial performance gains only for frequency bands which have a high duty cycle i.e., are highly utilized.

\subsection{Conclusion}

In this work, we presented analytical and simulation results for a general setup, involving multiple CR and PU links. Our results clearly show that significant performance gains can be obtained by incorporating location awareness, during the formulation of the resource management strategy. In addition, we proposed two novel algorithms with 


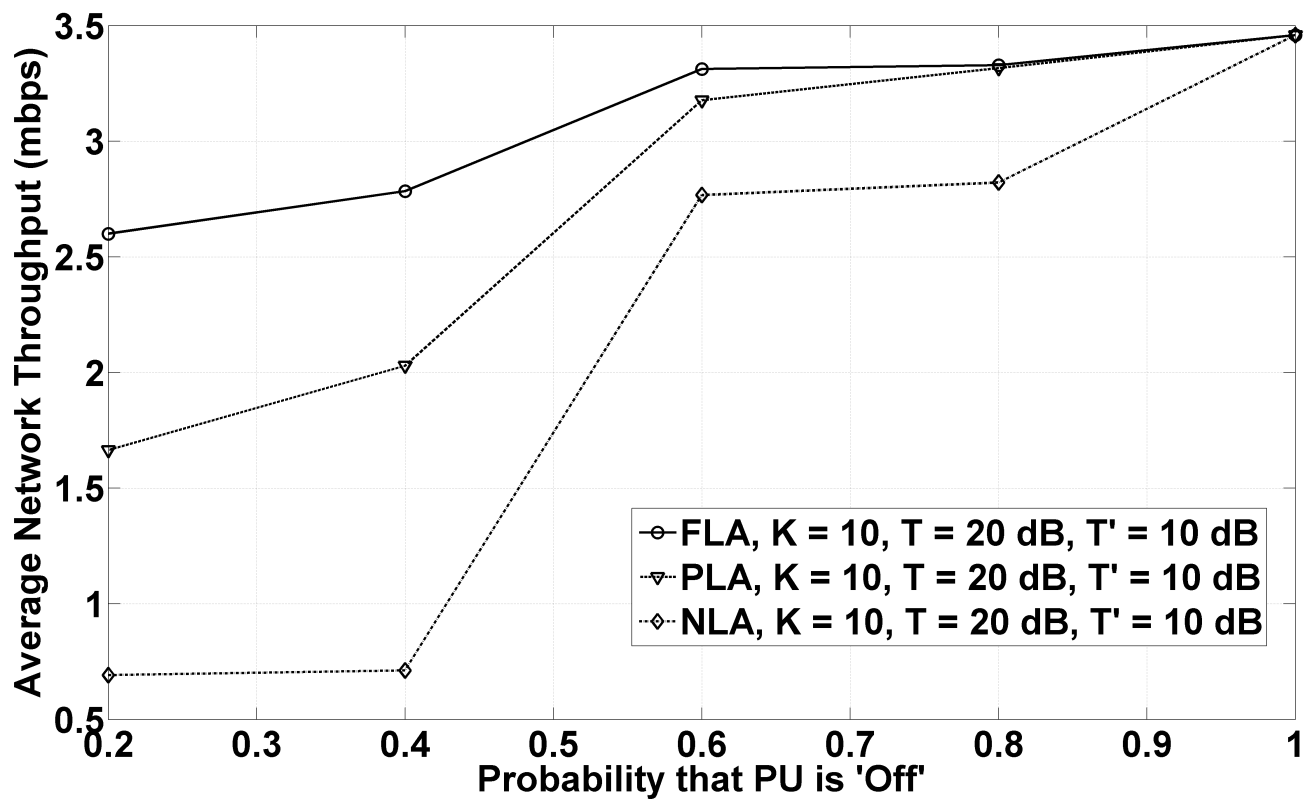

Figure 5.21 - Impact of PU duty cycle on CR network throughput for FLA, PLA and NLA scenarios.

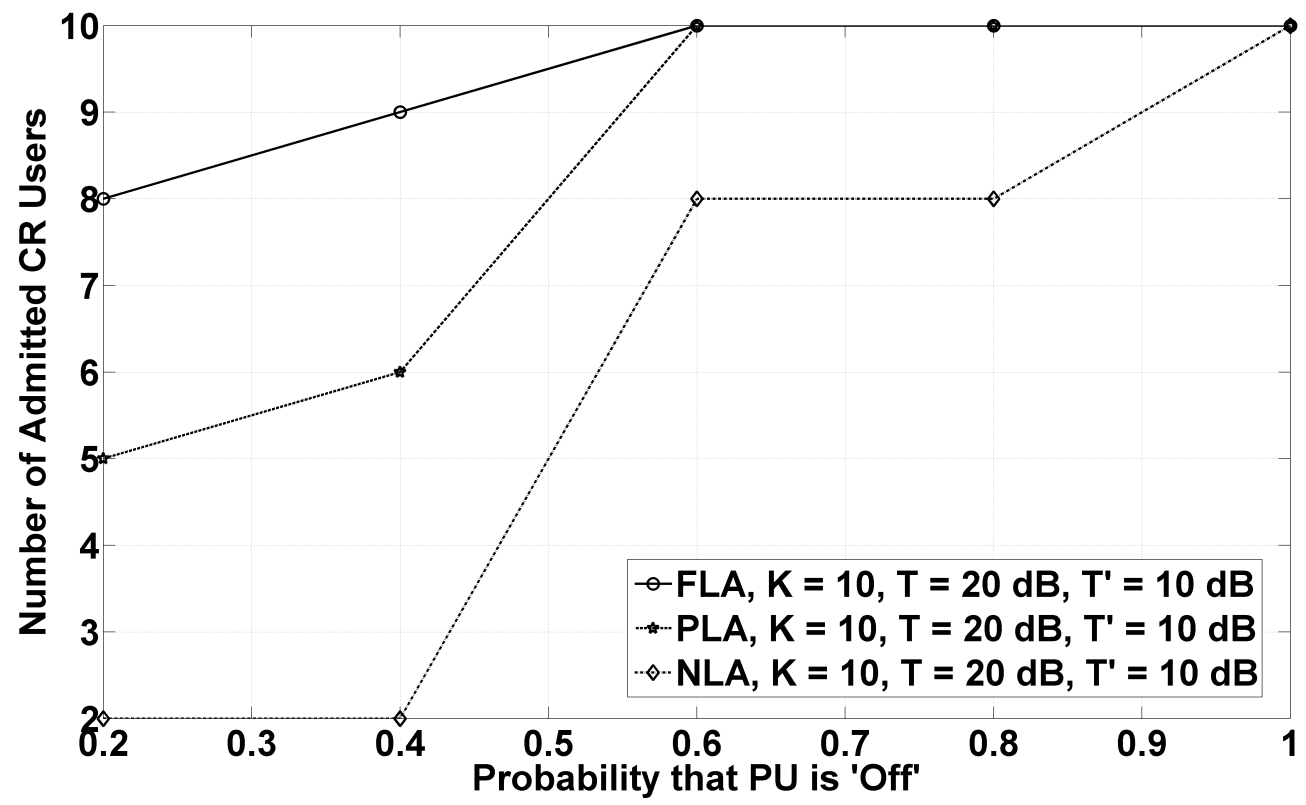

Figure 5.22 - Impact of PU duty cycle on number of admitted CR users for FLA, PLA and NLA scenarios. 
polynomial complexity, to solve the formulated resource management problems. 


\section{Chapter 6}

\section{Conclusions and Future Work}

The aim of this work was to study resource management in overlaid Cognitive Radio Networks. Specifically, we formulated and analyzed analyzed resource management strategies for a multi user scenario involving multiple primary and CR links. Further, we analyzed the impact of location awareness on network performance of the overlaid CR networks using average network throughput and number of admitted CR users as parameters.

\subsection{Major Research Contributions}

The main research contributions of this work are:

- The impact of incorporating location awareness during the formulation of resource management strategy for single CR user is analyzed, by considering 2 extreme cases: FLA and NLA. Further, impact of system parameters and propagation environment on overlaid CRN performance is examined. In addition, analytical expressions governing the successful coexistence of CRs and PUs are presented.

- Building upon previous results, the analysis is extended to a multi PU and CR user case. Here, the resource management problem is formulated as a mathematical optimization problem, with variables denoting transmission power and frequency channel assignments. Specifically, we focus on two key problems in system design: Maximization of Admitted Users and Sum Rate Maximization. In order to contrast and compare effect of degree of location awareness on CRN performance we consider three cases: FLA, PLA and NLA. Since the formulated optimization problems are NP hard, due to presence of binary variables we propose novel graph based algorithms for optimally solving the formulated optimization problems. 
- Further, for the PLA scenario we propose a novel technique for Primary Receiver (PR) localization using prior knowledge of receiver sensitivity. The proposed technique can be very useful in estimating location of passive PRs i.e., receivers that are always or mostly listening, such as TV receivers.

- Our Work clearly identifies scenarios where location awareness can prove to be a useful asset, along with scenarios where location awareness does not offer justifiable advantages and thus can be relinquished.

\subsection{Future Work}

The following relevant research topics are of importance and deserve further investigation:

- Multi-Channel Allocation: We have assumed that each CR pair is allocated exactly one primary channel upon admission. Although this model simplifies the analysis, it will be interesting to investigate scenario where CR pairs are allocated more than one primary channel upon admission.

- Distributed Resource Management: Here we have assumed the presence of a central spectrum allocation mechanism, which receives CR requests and is responsible for formulating the resource management strategy. Although this assumption simplifies the analysis significantly. However, when the scale of the CR network increases having a centralized structure may longer be feasible. Therefore, it would interesting to devise distributed resource management algorithms.

- Resource Management for Non Contiguous Bands: In our work we have assumed that all primary channels have similar characteristics i.e., equal bandwidth $W$ and similar propagation characteristics. However, in reality a CR may have access to primary channels varying in bandwidth, propagation characteristics etc. Therefore, it would be interesting to investigate resource management issues under such scenarios. 


\section{Bibliography}

[1] M. Vilimpoc and M. McHenry, "Dupont circle spectrum utilization during peak hours," tech. rep., The New America Foundation and The Shared Spectrum Company, 2003.

[2] Q. Zhao and B. Sadler, "A survey of dynamic spectrum access," IEEE Signal Processing Magazine, vol. 24, pp. 79 -89, May 2007.

[3] T. Yucek and H. Arslan, "A survey of spectrum sensing algorithms for cognitive radio applications," IEEE Communications Surveys Tutorials, vol. 11, no. 1, pp. 116 $-130,2009$.

[4] Y.-C. Liang, S. Sun, X. Peng, and F. Chin, "Tutorial 2: Emerging wireless standards for wran, wifi, wimedia and zigbee," in 10th IEEE Singapore International Conference on Communication systems (ICCS 2006), pp. 27 -29, oct. 2006.

[5] R. Coase, "The federal communications commission," Journal of Law and Economics, pp. 1-40, 1959.

[6] K. T. Kim and S. K. Oh, "Cognitive ad-hoc networks under a cellular network with an interference temperature limit," in 10th International Conference on Advanced Communication Technology, 2008., vol. 2, pp. 879 -882, feb. 2008.

[7] J. M. III, "Cognitive radio for flexible mobile multimedia communications.," MONET, vol. 6, no. 5, pp. 435-441, 2001.

[8] S. Yarkan and H. Arslan, "Exploiting location awareness toward improved wireless system design in cognitive radio," IEEE Communications Magazine, vol. 46, pp. $128-136$, January 2008.

[9] S. Haykin, "Cognitive radio: brain-empowered wireless communications," IEEE Journal on Selected Areas in Communications, vol. 23, pp. 201 - 220, feb. 2005. 
[10] P. Jia, M. Vu, and T. Le-Ngoc, "Location-aware cognitive sensing for maximizing network capacity," in 2009 Conference Record of the Forty-Third Asilomar Conference on Signals, Systems and Computers, pp. 78 -82, nov. 2009.

[11] N. Yi, Y. Ma, and R. Tafazolli, "Underlay cognitive radio with full or partial channel quality information," International Journal of Navigation and Observation, vol. abs/1009.2927, 2010.

[12] T. Yucek and H. Arslan, "A survey of spectrum sensing algorithms for cognitive radio applications," IEEE Communications Surveys Tutorials, vol. 11, pp. 116 130 , quarter 2009.

[13] H. Urkowitz, "Energy detection of unknown deterministic signals," Proceedings of the IEEE, vol. 55, pp. 523 - 531, April 1967.

[14] H. Tang, "Some physical layer issues of wide-band cognitive radio systems," in First IEEE International Symposium on New Frontiers in Dynamic Spectrum Access Networks, 2005., pp. 151 -159, Nov. 2005.

[15] Z. Ye, J. Grosspietsch, and G. Memik, "Spectrum sensing using cyclostationary spectrum density for cognitive radios," in 2007 IEEE Workshop on Signal Processing Systems, pp. 1 -6, Oct. 2007.

[16] J. Renard, J. Verlant-Chenet, J.-M. Dricot, P. De Doncker, and F. Horlin, "Higherorder cyclostationarity detection for spectrum sensing," EURASIP Journal on Wireless Communications and Networking, vol. 2010, pp. 3:1-3:11, January 2010.

[17] J. G. Proakis and D. K. Manolakis, Digital Signal Processing (4th Edition). Prentice Hall, 2006.

[18] A. Ghasemi and E. S. Sousa, "Spectrum sensing in cognitive radio networks: the cooperation-processing tradeoff," Journal Wireless Communications and Mobile Computing, vol. 7, pp. 1049-1060, November 2007.

[19] Z. Tian and G. Giannakis, "Compressed sensing for wideband cognitive radios," in IEEE International Conference on Acoustics, Speech and Signal Processing, 2007., vol. 4, pp. IV-1357 -IV-1360, April 2007.

[20] P. Jia, M. Vu, and T. Le-Ngoc, "Location-aware cognitive sensing for maximizing network capacity," in Proceedings of the 43rd Asilomar conference on Signals, systems and computers (Asilomar'09), (Piscataway, NJ, USA), pp. 78-82, IEEE Press, 2009. 
[21] B. A. Fette and B. Fette, Cognitive Radio Technology (Communications Engineering). Newnes, 2006.

[22] A. T. Hoang, Y.-C. Liang, and M. Islam, "Power control and channel allocation in cognitive radio networks with primary users' cooperation," IEEE Transactions on Mobile Computing, vol. 9, pp. 348 -360, March 2010.

[23] W. Su, J. Matyjas, and S. Batalama, "Active cooperation between primary users and cognitive radio users in cognitive ad-hoc networks," in 2010 IEEE International Conference on Acoustics Speech and Signal Processing (ICASSP), pp. 3174 -3177, March 2010.

[24] S. Kim, H. Jeon, H. Lee, and J. S. Ma, "Information networking. towards ubiquitous networking and services," in Information Networking. Towards Ubiquitous Networking and Services (T. Vazão, M. M. Freire, and I. Chong, eds.), ch. Robust Transmission Power and Position Estimation in Cognitive Radio, pp. 719-728, Berlin, Heidelberg: Springer-Verlag, 2008.

[25] R. Martin and R. Thomas, "Algorithms and bounds for estimating location, directionality, and environmental parameters of primary spectrum users," IEEE Transactions on Wireless Communications, vol. 8, pp. 5692 -5701, November 2009.

[26] W. Wang, F. Li, Y. Zhang, S. Wang, and Y. Wang, "Direct primary receiver detection with frequency mapping in cognitive radio," in IEEE International Conference on Communications Technology and Applications (ICCTA '09), pp. 493 -499, Oct. 2009.

[27] B. Wild and K. Ramchandran, "Detecting primary receivers for cognitive radio applications," in First IEEE International Symposium on New Frontiers in Dynamic Spectrum Access Networks, 2005, pp. 124 -130, Nov. 2005.

[28] S. Park, L. Larson, and L. Milstein, "An rf receiver detection technique for cognitive radio coexistence," IEEE Transactions on Circuits and Systems II: Express Briefs, , vol. 57, pp. 652 -656, aug. 2010.

[29] Y. Zhao, L. Morales, J. Gaeddert, K. Bae, J.-S. Um, and J. Reed, "Applying radio environment maps to cognitive wireless regional area networks," in 2nd IEEE International Symposium on New Frontiers in Dynamic Spectrum Access Networks, 2007., pp. 115 -118, April 2007.

[30] L. Liu, Z. Li, and C. Zhou, "Backpropagation-based cooperative localization of primary user for avoiding hidden-node problem in cognitive networks," International Journal of Digital Multimedia Broadcasting, 2010. 
[31] Q. Gu, RF System Design of Transceivers for Wireless Communications. Secaucus, NJ, USA: Springer-Verlag New York, Inc., 2006.

[32] L.-C. Wang and A. Chen, "Effects of location awareness on concurrent transmissions for cognitive ad hoc networks overlaying infrastructure-based systems," IEEE Transactions on Mobile Computing, vol. 8, pp. 577-589, May 2009.

[33] Y. Hou, Y. Shi, and H. Sherali, "Optimal spectrum sharing for multi-hop software defined radio networks," in 26th IEEE International Conference on Computer Communications (INFOCOM 2007), pp. 1 -9, May 2007.

[34] E. Arikan, "Some complexity results about packet radio networks (corresp.)," IEEE Transactions on Information Theory, vol. 30, pp. 681 - 685, jul 1984.

[35] D. Jungnickel, Graphs, Networks and Algorithms. Springer Publishing Company, Incorporated, 3rd ed., 2007.

[36] W. Guo and X. Huang, "Maximizing throughput for overlaid cognitive radio networks," in IEEE Military Communications Conference, 2009. MILCOM 2009., pp. $1-7$, Oct. 2009.

[37] C. Papadimitriou and K. Steiglitz, Combinatorial Optimization: Algorithms and Complexity. Dover Publications, 1998.

[38] J. Munkres, "Algorithms for the assignment and transportation problems," Journal of the Society for Industrial and Applied Mathematics, vol. 5, no. 1, pp. pp. 32-38, 1957.

[39] H. A. B. Salameh, "Throughput-oriented channel assignment for opportunistic spectrum access networks," Mathematical and Computer Modelling, vol. In Press, Corrected Proof, 2010. 